University of Montana

ScholarWorks at University of Montana

Graduate Student Theses, Dissertations, \&

Professional Papers

2001

\title{
Financial cost-benefit analysis of a health promotion program for individuals with mobility impairments
}

Catherine Ipsen

The University of Montana

Follow this and additional works at: https://scholarworks.umt.edu/etd

Let us know how access to this document benefits you.

\section{Recommended Citation}

Ipsen, Catherine, "Financial cost-benefit analysis of a health promotion program for individuals with mobility impairments" (2001). Graduate Student Theses, Dissertations, \& Professional Papers. 6327. https://scholarworks.umt.edu/etd/6327

This Thesis is brought to you for free and open access by the Graduate School at ScholarWorks at University of Montana. It has been accepted for inclusion in Graduate Student Theses, Dissertations, \& Professional Papers by an authorized administrator of ScholarWorks at University of Montana. For more information, please contact

scholarworks@mso.umt.edu. 


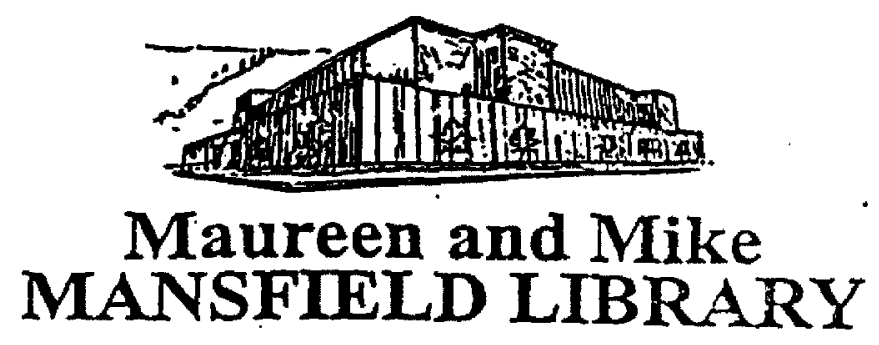

The University of Montana

Permission is granted by the author to reproduce this material in its entirety, provided that this material is used for scholarly purposes and is properly cited in published works and reports.

**Please check "Yes" or "No" and provide signature**

Yes, I grant permission

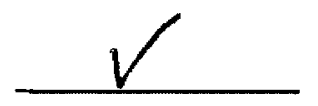

No, I do not grant permission

Author's Signature: (ath ol

Date: $2 / 20 / 01$

Any copying for commercial purposes or financial gain may be undertaken only with the author's explicit consent. 


\title{
A FINANCIAL COST-BENEFIT ANALYSIS OF A HEALTH PROMOTION PROGRAM FOR INDIVIDUALS WITH MOBILITY IMPAIRMENTS
}

\author{
by \\ Catherine Ipsen \\ B.S. Occidental College, 1987 \\ presented in partial fulfilment of the requirements for the degree of \\ Masters of Economics \\ The University of Montana \\ February 2001
}
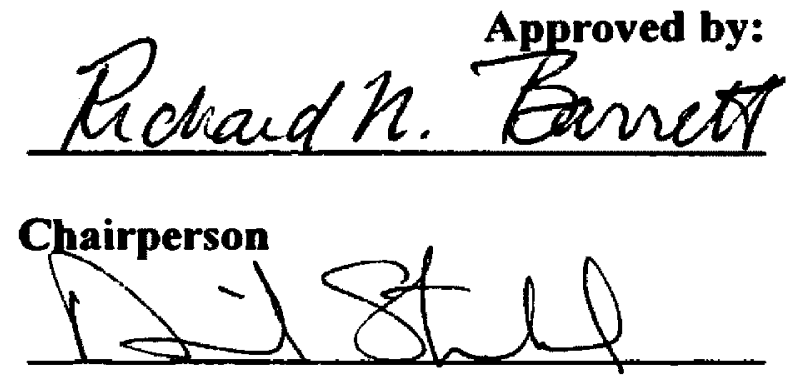

Dean, Graduate School $02 / 20 / 01$

Date 


\section{All rights reserved}

\section{INFORMATION TO ALL USERS}

The quality of this reproduction is dependent upon the quality of the copy submitted.

In the unlikely event that the author did not send a complete manuscript and there are missing pages, these will be noted. Also, if material had to be removed, a note will indicate the deletion.

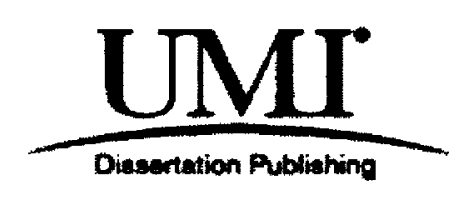

\section{UMI EP37128}

Published by ProQuest LLC (2013). Copyright in the Dissertation held by the Author.

Microform Edition @ ProQuest LLC.

All rights reserved. This work is protected against unauthorized copying under Title 17, United States Code

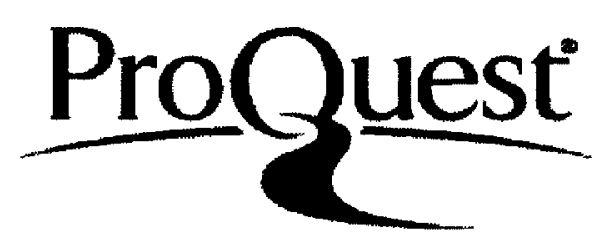

ProQuest LLC.

789 East Eisenhower Parkway

P.O. Box 1346

Ann Arbor, MI 48106 - 1346 
A Financial Cost-Benefit Analysis of a Health Promotion Program for Individuals with Mobility Impairments

Director: Richard Barrett.<smiles>[Mg][Mg]</smiles>

There is a growing body of literature which identifies health promotion as both effective in terms of health outcomes and cost-effective compared to treatment alternatives. Yet, health promotion interventions are typically not reimbursed by third party payers such as Medicaid, Medicare, or private insurance. This is a problem for individuals with disabilities who have significant health care utilization costs but cannot pay for, and benefit from, health promotion programs.

The purpose of this study was to show the net benefits of the Living Well with a Disability health promotion program from the perspective of a third party payer. Net benefits were defined as reductions in health care utilization costs minus program implementation costs. Self report health care utilization data for 188 participants of the Living Well health promotion program were collected at five times (baseline, immediate post-intervention, 2 months post-intervention, 4 months post-intervention, and one-year post-intervention). Additional data, collected at 2 months prior to intervention for 79 participants, served as an extended baseline control measure.

The Living Well program resulted in positive net benefits within the first six months of Living Well implementation for the entire cohort, trimmed data, and a high medical use sub-sample. Sensitivity analyses were conducted for a range of medical costs, using Medicare cost estimates as the base, and $70 \%$ and $130 \%$ of Medicare costs to construct a range. Using base health care unit costs, the net benefits were $\$ 2,729$ per participant in the entire study cohort, $\$ 589$ per participant for the trimmed data set, and $\$ 1,484$ per participant for the high medical use group. These results provide a clear message to third party payers about the financial benefits of supporting health promotion programs for individuals with disabilities. 


\section{Acknowledgments}

There are not many graduate students who have been enrolled in the same program over a three decade span. Yet, I have managed to spend part of the 80's, 90's and new millennium working to fulfill my graduate requirements for a Master's in Economics. While this untimely schedule is no feather in my cap, it has given me a long list of people to thank.

First, there are several people in the Economics Department who have played a part in my readmittance into the program. Dick Barrett, my thesis chair and good friend, has managed to keep me connected with the program one mile at a time. Before assuming his role as thesis chair, Dick ran, skied, and biked thousands of miles with me, gently prodding me back to my thesis work. Doug Dalenberg, in addition to serving on my thesis committee, had to do some fancy dancing to get me readmitted into the graduate school. This was no easy feat considering that the class requirements and credit systems have changed since my initial enrollment. Becky Hofstad has remained a great friend throughout. It is hard to believe that her college-aged son was in second grade when I began my graduate work and lived in her home.

Craig Ravesloot, a third thesis committee member and my supervisor, and Tom Seekins, Director of the Research and Training Center at the Rural Institute on Disabilities, were instrumental in focusing my efforts. Not only did they provide work time and resources to complete my thesis, but also the project data. Additionally, several people at the Rural Institute have helped this effort including Ann Szalda-Petree, Lisa Brennan, and Diana Spas....master editors, all. Finally, thanks to Steve Seninger, the final member of my thesis committee, who provided valuable resources and cost figures for my paper.

Each member of my family, in his or her own way, has unselfishly supported this effort. Thanks especially to my husband, Jeff, who spent many weekends and nights alone with our two children, Ellen and Eric, while I came into work. 


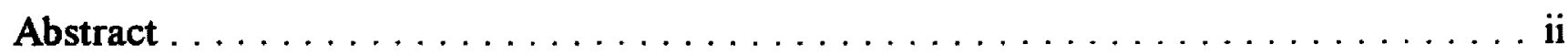

Acknowledgments $\ldots \ldots \ldots \ldots \ldots \ldots \ldots \ldots \ldots \ldots \ldots \ldots \ldots \ldots \ldots \ldots \ldots$ iii

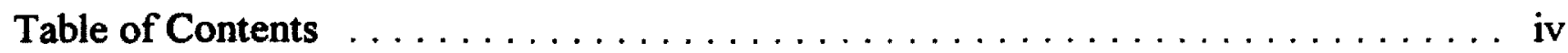

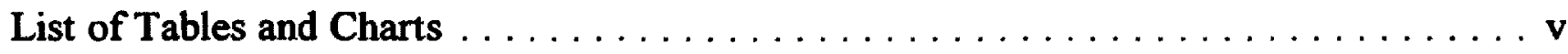

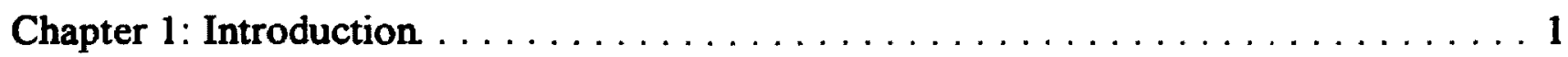

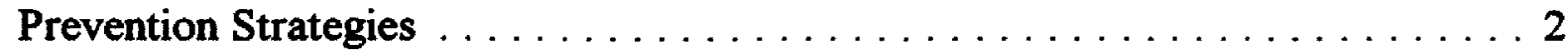

Health Promotion for Individuals with Disabilities $\ldots \ldots \ldots \ldots \ldots \ldots \ldots 5$

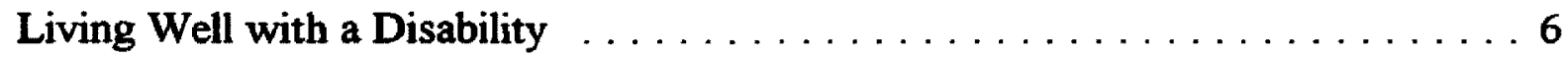

A Cost-Benefit Analysis of Living Well $\ldots \ldots \ldots \ldots \ldots \ldots \ldots \ldots$

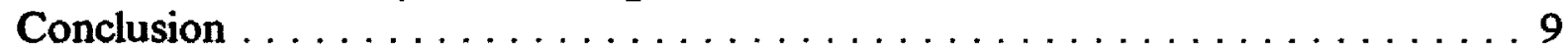

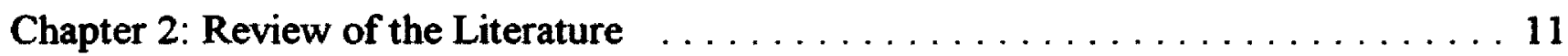

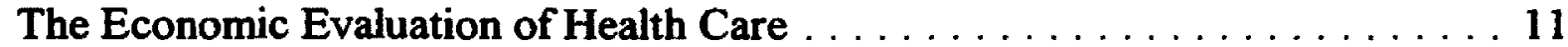

Current Studies of Health Promotion for Individual with Disabilities . . . . 21

Current Studies of Health Promotion for Individuals without Disabilities . . . 28

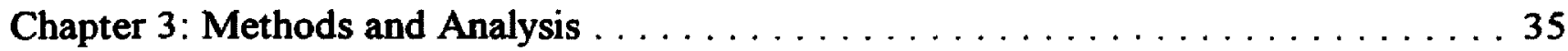

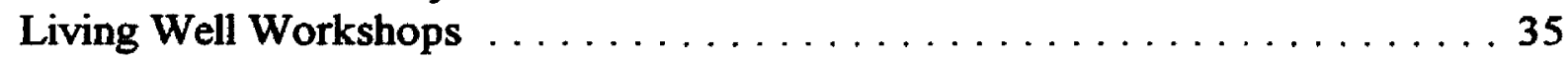

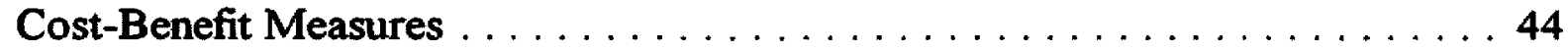

Chapter 4: Cost-Benefit Analysis - Results $\ldots \ldots \ldots \ldots \ldots \ldots \ldots \ldots \ldots \ldots \ldots$

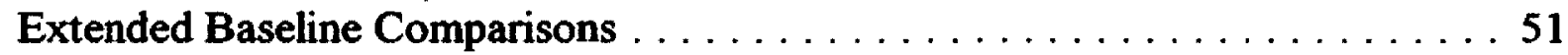

The Cost-Benefit Analysis . . . . . . . . . . . . . . . . . . . 59

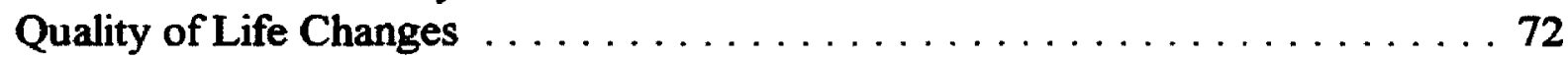

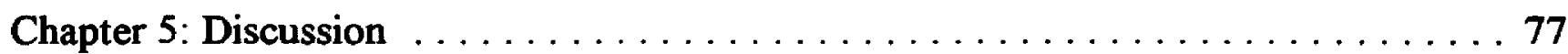

References $\ldots \ldots \ldots \ldots \ldots \ldots \ldots \ldots \ldots \ldots \ldots \ldots \ldots \ldots \ldots \ldots$

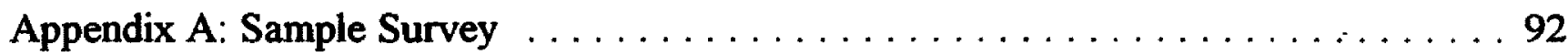

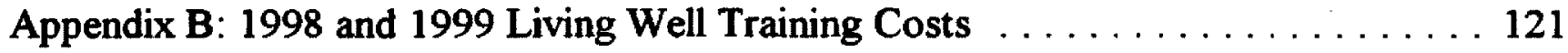




\section{List of Tables and Charts}

Chapter 2: Review of the Literature

Table 1: An Example of Marginal Net Benefit Calculations $\ldots \ldots \ldots \ldots 14$

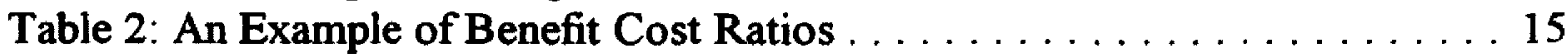

Chapter 3: Methods and Analysis

Table 3: Living Well Implementation Dates $\ldots \ldots \ldots \ldots \ldots \ldots \ldots \ldots$

Table 4: Living Well Recruitment Methods $\ldots \ldots \ldots \ldots \ldots \ldots \ldots \ldots$

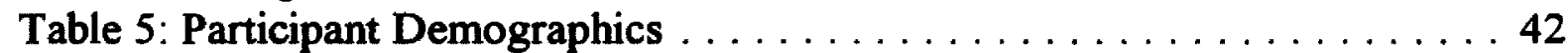

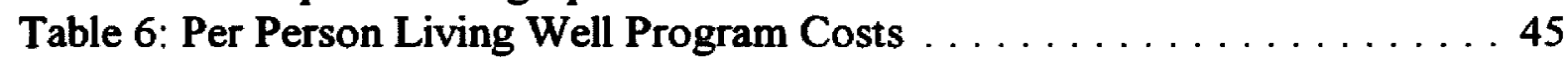

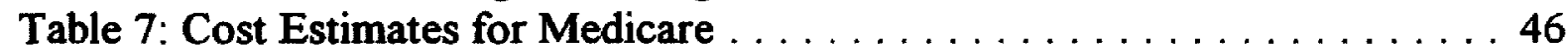

Table 8: State Medicaid Reimbursement Compared to National Average . . . . 48

Table 9: Low, Medium, and High Cost Estimates . . . . . . . . . . . 49

Chapter 4: Cost Benefit Analysis - Results

Table 10: Extended Baseline Comparison (Survey A and Survey B) . . . . . 54

Table 11: Demographic Comparisons Between Extended Baseline and Comparison . ................... 56

Table 12: Health Characteristic Comparisons Between Extended

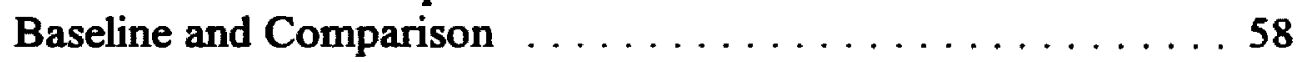

Table 13: Mean Health Care Expenditures . . . . . . . . . . . 61

Table 14: Net Benefits - Entire Cohort of 188 Participants . . . . . . . 61

Table 15: Net Benefits - Trimmed Data Set $\ldots \ldots \ldots \ldots \ldots \ldots \ldots \ldots 6$

Table 16: Net Benefits - High Use Participants . . . . . . . . . 62

Table 17: Paired Sample Tests - Immediate Pre (B) to Post Intervention Points (C, D, \& E) . . . . . . . . . . . . . . 63

Table 18: Immediate Pre and One-Year Post Health Care Utilization Comparisons . . . . . . . . . . . . . . . 66

Table 19: COST1 - Mean, Standard Deviation, and Variance . . . . . . . . 67

Table 20: Program Outcome Confidence Intervals $\ldots \ldots \ldots \ldots \ldots 6$

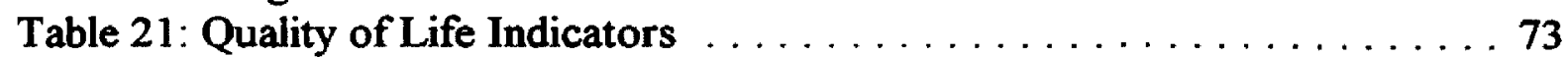

Table 22: Quality of Life Indicators - Equality of Means Tests . . . . . . . 74

Chart 1: $95 \%$ Confidence Intervals for Cohort Data . . . . . . . . . 71

Chart 2: 95\% Confidence Intervals for Trimmed Data . . . . . . . . 71

Chart 3: $95 \%$ Confidence Intervals for High Medical Use Data $\ldots \ldots \ldots \ldots 71$ 


\section{Chapter 1: Introduction}

In the face of significant increases in the price of health care, health promotion has been proposed as a viable way to contain costs. There is a growing body of literature which identifies health promotion as both effective in terms of health outcomes and costeffective in relation to treatment alternatives (Pelletier, 1996).

To date, health promotion efforts and subsequent studies have generally been conducted in work-site settings and have focused on work-site interventions. Both employers and health care providers cite the work-site as the best place to systematically reach the adult population (Pelletier, 1996; Shi, 1993). Clearly, this focus on health promotion for employed adults leaves a significant portion of the population lacking health benefits associated with health promotion activities.

Represented in this excluded population are many individuals with disabilities who do not work and the elderly. Although health promotion efforts often overlook people in these groups, particularly those who suffer from chronic conditions, they can have significant health care costs (Marge, 1988). According to Hoffman, et al. (1996), people with activity limitations due to chronic conditions constitute $17 \%$ of the general population, but account for $47 \%$ of medical expenditures. Moreover, it has been documented that $75 \%$ of US health care expenditures accrue from non-institutionalized individuals with at least one chronic condition (Lorig, et al., 1999).

In their reviews of studies about health promotion and disease prevention programs at the work-site, Pelletier (1996) and Kaman \& Patton (1992) found that the most significant health cost savings are experienced by a small number of high-risk 
individuals. This suggests that exclusion of individuals with chronic conditions who are not able to work is a significant oversight in light of the potential cost reductions and health benefits associated with health promotion activities. Although there are a growing number of federal agencies that recognize and support development of health promotion programs for individuals with disabilities, economic cost and feasibility studies are rare (Rimmer, 1999; Taylor, Baranowski, \& Young, 1998).

\section{Prevention Strategies}

Health promotion is described as a behavioral prevention strategy. In general, prevention strategies fall into three categories: clinical, behavioral, and environmental. Clinical prevention programs, often referred to as preventative medicine, generally occur in a clinical setting, are brief in duration, and have passive patient involvement. Examples include vaccinations, health screenings, and treatment monitoring. Conversely, behovioral interventions rely on patient action to affect health outcomes. Although a clinician may prescribe a prevention plan, the individual must modify his/her own behavior over time to reap health benefits. Examples of behavioral interventions include changes in diet, exercise, or other risk behaviors such as smoking and drinking. Environmental prevention is typically imposed by legislation or established rules and protocols. This includes actions such as fluorinated public water and lead abatement legislation (Haddix, Teutsch, Shaffer, Dunet, 1996).

Although a full range of health promotion/disease prevention programs have been found to significantly reduce long and short-term health care costs, only 3 percent of health care expenditures are allocated to promotion/prevention efforts (Phillips \& 
Holtgrave, 1997). This phenomenon is surprising in the face of a growing body of literature which documents the significant cost savings associated with promotion/prevention efforts (Pelletier, 1996).

Historically, the health care delivery system was constructed to respond to acute illness, and this is still largely true today. This is one of the main factors contributing to the under-production of prevention programs. Not only is insurance coverage based on this acute model, but acute care has defined the entire health care system, including how research dollars are spent (Hoffman, Rice, \& Sung, 1996). Without adequate research in the area of prevention, the transition from pilot prevention programs into established standards of care is delayed.

Moreover, unlike acute interventions which have clear outcomes, health promotion/disease prevention efforts are typically removed from the health outcomes they are targeted to impact. This is particularly true for behavioral health promotion interventions which require (1) lengthy time horizons to see results and (2) a degree of patient compliance to deliver expected outcomes which may be difficult to directly measure. Health promotion/disease prevention programs require more rigorous defense than medical treatments because program costs are incurred with less certainty regarding future health outcomes (Keeler, Manning, Newhouse, Sloss, \& Wasserman, 1989).

In the case of behavioral interventions, questions about strength and causality must also be addressed (Stachtchenko, \& Jenicek, 1990; Keeler, Manning, Newhouse, Sloss, \& Wasserman, 1989). For instance, although risk factors such as diet and exercise are associated with future disease, there is no clear evidence about the extent to which 
eliminating or reducing these risk factors results in a reduction in health problems (Barry \& DeFriese, 1990). When intervention benefits are clear, they can be translated into economic terms and easily incorporated into the decision making process. However, in the case of modifying risky behavior, one must predict long-term outcomes from current health behaviors. Broadly focused health promotion programs, like Living Well with a Disability (Ravesloot, et al. 1998), compound this problem since they are designed to impact a variety of health outcomes through a range of lifestyle changes. With each additional lifestyle change, it becomes more difficult to determine the extent to which different health factors contribute to specific health outcomes.

Review of the literature corroborates this difficulty. There are several cost-benefit studies of prevention strategies linked to specific outcomes (i.e. screenings for cancer, vaccinations for specific diseases, and bicycle helmet intervention programs for head injuries), but the literature is sparse for broad lifestyle modifications. In an article summarizing the literature on prevention strategies associated with 19 areas of chronic and infectious disease, Messonier, et al. (1999) do not cite one study which examines a broad-based health promotion intervention designed to modify multiple behaviors and affect multiple health outcomes.

Clearly, introduction of health promotion strategies into the existing acute care service model requires persuasive arguments about the net benefit outcomes. If it is a goal to extend health promotion to individuals with disabilities who typically have high medical needs or are at a higher risk of experiencing certain conditions, a model must be developed and examined beyond the typical work-site setting. 


\section{Health Promotion for Individuals with Disabilities}

There are several additional issues associated with development and delivery of health promotion programs for individuals who have significant disabilities or chronic conditions. First, individuals with disabilities face barriers which add costs to program interventions and impact on-going health maintenance. These barriers include lack of transportation, programmatic inaccessibility, pain, and fatigue. Barriers increase the cost of health promotion through recruitment and maintenance issues as well as influencing the program outcomes (Rimmer, 1999; Kinne, Patrick, \& Maher, 1999).

Financial issues can also be a significant constraint (Seekins \& Ravesloot, 2000). The 1998 Chartbook on Work and Disability indicates that the percentage of adults (aged 16 to 64 ) with mobility impairments not working is $72.5 \%$ (Stoddard, Jans, Ripple, \& Kraus, 1998). As a group, individuals with disabilities are the poorest of the poor and do not have the financial resources to pay for health promotion services.

At the same time, health promotion and disease prevention programs have not been financially supported by third-party payers such as insurance companies, Medicaid, and Medicare (Guo, Gibson, Gropper, Oswald, \& Barker, 1998; Hoffman, Rice, \& Sung, 1996). While individuals with disabilities are in a state of significant financial risk with per capita medical costs that are 3 times higher than individuals without chronic conditions (Hoffman, Rice, \& Sung, 1996), third party payers overlook prevention programs as a viable way to reduce costs. Although some managed care companies offer lower out-of-pocket expenses and coverage for health promotion/disease prevention services, they have historically excluded populations with high medical utilization rates 
from participation all together (Hoffman, Rice, \& Sung, 1996).

Although third party payers reap the benefits of health promotion activities through reduced medical utilization, it is often the individual or some other party like the employer, who bears the participation costs. In the case of employer sponsored programs, reduced costs may be realized in lower group insurance premiums. At the individual level, out-of-pocket expenses may be offset by reduced spending on medical goods and services. For individuals with disabilities who have no disposable income, however, the system breaks down. Without employer support to cover prevention programs or the individual resources to bear costs, many significant medical health care users are excluded from health promotion programs.

Clearly, if health promotion programs are going to be adopted by individuals with disabilities, insurance carriers must reduce this economic barrier to participation through expanded coverage. Because health promotion programs and associated studies have typically focused on work-site interventions, however, third party payers may be unaware of the financial benefits of health promotion that targets individuals with disabilities and chronic conditions.

\section{Living Well with a Disability}

In response to the need for a health promotion program aimed specifically at individuals with disabilities, Living Well with a Disability was developed to reduce secondary conditions that occur as a result of, or in conjunction with, a primary disability (Ravesloot, et al., 1998). For instance, a spinal cord injury may result in several secondary conditions, including pressure sores, urinary tract infections, osteoporosis, and 
depression. In 1997, the Centers for Disease Control (CDC) funded the development, implementation, evaluation, and analysis of Living Well to determine the effectiveness of this type of health promotion strategy in reducing the occurrence and severity of secondary conditions experienced by individuals with disabilities.

The Living Well health promotion intervention uses both cognitive and behavioral approaches to improve secondary conditions for individuals with mobility impairments. Through eight weekly training sessions, Living Well program participants are encouraged to make positive behavioral changes through increased knowledge, communication skills, coping strategies, and self-advocacy skills. The program helps participants identify how healthy behaviors contribute to the attainment of long-term goals. Modifications of health behaviors are then established as a means to goal attainment.

Living Well is unique both in the delivery system (lay trainers working through Centers for Independent Living) and focus (health promotion from a disability framework). The Living Well program has been delivered 34 times at 9 different Independent Living Centers during the past two years. During this implementation phase, participants completed a series of surveys to examine program efficacy, efficiency and cost effectiveness. Since the target population is characteristically economically disadvantaged, grant funding was necessary to implement Living Well without passing costs on to program participants. A major portion of this support was to determine whether the cost of implementing Living Well is warranted in terms of health and economic benefits. 


\section{A Cost-Benefit Analysis of the Living Well with a \\ Disability Health Promotion Program}

This study presents a cost-benefit analysis of the Living Well program from the perspective of a third party payer such as Medicaid, Medicare or private insurance. Since individuals with disabilities typically place a significant encumbrance on public and private insurance carriers through high medical utilization rates, it seems logical that insurance programs would be willing to bear these health promotion costs if they can be shown to reduce overall medical expenses.

Evaluation of health care programs can yield significantly different results based on the viewpoint adopted (Drummond, Stoddart, \& Torrance, 1987). For Living Well, these interests might be those of the program participant, health promotion provider, third party payer, government, or society as a whole. If costs are to be assumed by insurance providers, however, it is important to demonstrate net savings from the program. Although the societal perspective provides the broadest description of program costs and benefits because it incorporates all analytic viewpoints, it is not relevant or convincing when decisions are made about providing or paying for specific prevention programs (Haddix, Teutsch, Shaffer, Dunet, 1996).

The viewpoint of the third party payer is also attractive given constraints of the Living Well survey data. For example, Living Well data is confined to a one year horizon and does not adequately answer questions about indirect costs to the consumer and their family/friends, out-of-pocket consumer medical expenses, health impacts on employment, hospital/care provider impacts, and potential changes in health care 
providers. Although these limitations present several measurement problems from a societal perspective, a focused analysis is possible. Living Well data does answer questions about direct health care utilization rates for program participants over a oneyear span, and as such, provides valuable information for third party payers who are interested in health promotion impacts. If direct health cost savings can be shown within the first year of program implementation, the strongest case for third party payer support is provided.

Using this short time horizon confines analysis to outcomes which are more directly impacted by behavior change. Behavioral changes like diet and exercise can immediately influence medical conditions such as mild depression (Stevens, Hillsdon, Thorogood, \& McArdle, 1998), seasonal affective disorder (Marge, 1988), urinary tract infections (Marge, 1988), high blood pressure (Stevens, Hillsdon, Thorogood, \& McArdle, 1998), and pressure sores (Marge, 1988). This connection is weaker, however, for long-term conditions such as heart disease, osteoporosis, and cancer, especially when questions of ongoing behavior maintenance are included. While a short time horizon excludes the full health impacts of lifestyle changes, immediate results can be reported with more confidence to third party payers. Additionally, the short time horizon shows net savings that are more likely to be realized by the third party payer who made the health promotion investment.

\section{Conclusion}

The following study is a cost-benefit analysis of a health promotion/disease prevention program designed specifically for individuals with disabilities. The analysis 
adopts the perspective of a third party payer and shows net benefits over a one-year span.

Chapter 2 presents a review of pertinent literature including an overview of cost models used in the evaluation of health care programs, specific economic evaluations of health promotion programs, and current wellness programs addressing the needs of individuals with mobility impairments. Chapter 3 provides a complete description of the Living Well program, including assessment of the costs and benefits measured in the model. The analysis of these costs and benefits is described in Chapter 4 . The final chapter addresses limitations and weaknesses of the cost-benefit study and discussion points for future inquiry. 


\section{Chapter 2. Review of the Literature}

This chapter consists of three sections, each of which discusses an important aspect of the extensive literature on the economic evaluation of health care programs. The first section covers general methodology of the economic evaluation of health care programs. The second section presents current studies of health promotion/disease prevention interventions specific to individuals with disabilities. The third section covers more broadly focused health promotion programs for the general population.

\section{The Economic Evaluation of Health Care}

Empirical evaluation of health promotion/disease prevention activities has grown dramatically in the past two decades. Faced with limited public health resources, it has become increasingly important to demonstrate good medical value for resources spent (Haddix, Teutsch, Shaffer, Dunet, 1996). Evaluation of medical programs, however, has proven to be difficult because medical prices do not reflect actual costs and the economic value of medical outcomes are difficult to measure.

On the cost side, prices for health care services are distorted and do not accurately reflect the cost of inputs. Government interventions and legislation shape medical service delivery and health care reimbursement rates to compensate for individuals who cannot pay for services (Haddix, Teutsch, Shaffer, Dunet, 1996). Prices for medical goods and services are negotiated through government insurance programs (i.e. Medicare and Medicaid), large private insurance agencies (i.e. Blue Cross/Blue Shield), and HMOs which exercise price control based on their size and financial strength (Haddix, Teutsch, Shaffer, Dunet, 1996). Medical charges are not representative of actual costs because 
cost-shifting occurs within provider agencies when (1) some services are charged at a higher rate to compensate for low profit margins in other service areas (Haddix, Teutsch, Shaffer, Dunet, 1996), and (2) the prices charged for services, particularly acute care, are adjusted in accordance with individual ability to pay. Obviously, it must be recognized that prices are an inaccurate proxy for the cost of medical services.

On the benefit side, it is difficult to attach a monetary value to health outcome measures. What is the value of an additional year of life, a lost limb, or a decrease in pain level? If health value is imputed on the basis of lost productivity or willingness to pay, distributional characteristics come to play (Andrich, Ferrario, \& Moi, 1998). In this scenario, a lower value would be attached to medical outcomes for economically disadvantaged subgroups such as women, people with disabilities, minorities, and the elderly who have lower income levels, and therefore, have lower productivity rates (Drummond, Stoddart, \& Torrance, 1987).

Adding to the difficulty of attaching a monetary value to health outcomes is the subjective value each person ascribes to certain health conditions. Consider a marathon runner and a photographer with similar earnings. It is probable that these two individuals would attach very different values to medical outcomes such as a knee replacement, enhanced eyesight, or reduced back pain.

Given the complexity associated with measuring health costs and outcomes, it is not surprising that a wealth of literature is devoted to the methodology of health care evaluation. In the literature there are three basic strategies proposed to address health care program evaluation: (1) cost benefit analysis, (2) cost-effectiveness analysis, and (3) 
cost-utility analysis. Although all three strategies use sensitivity analysis (a range of values) as a mechanism to deal with the uncertainty of costs and outcomes, these strategies have different strengths and weaknesses to address the measurement difficulties described.

\section{Cost Benefit Analysis}

Cost benefit analysis (CBA) is grounded in traditional economic welfare theory and evaluates all costs and outcomes in monetary units. In CBA, health outcomes are typically evaluated using either a willingness to pay or human capital approach where health is assigned a value based on an individual's productive value (Gold, Siegel, Russell, \& Weinstein, 1996). Using the human capital approach as an example, health outcomes are measured in terms of extended work life. Cost-benefit analysis can either compare programs based on net benefits or in terms of benefit/cost ratios.

Within $\mathrm{CBA}$, marginal net benefit is defined as $\mathrm{NBi}=(\mathrm{Bi}-\mathrm{Bb})-(\mathrm{Ci}-\mathrm{Cb})$, where $\mathrm{Bi}$ is benefits from the intervention, $\mathrm{Bb}$ is benefits from the baseline, $\mathrm{Ci}$ is costs of the intervention, and $\mathrm{Cb}$ is costs from the baseline. Marginal net benefit calculations are attractive when there are several competing interventions because calculations can easily be ranked to show the highest return for available resources (Birch \& Donaldson, 1987). Consider an example of five different interventions and a budget constraint of $\$ 150$. Based on marginal net benefit calculations, it is easy to rank the programs and determine which program, or combination of programs, provides the most benefits. Table 1 provides a comparison of five intervention strategies. 
Table 1: An Example of Marginal Net Benefit Calculations

\begin{tabular}{||l|c|c|c|c|c||}
\hline & Baseline & Program A & Program B & Program C & Program D \\
\hline \hline Program Costs & $\$ 20$ & $\$ 75$ & $\$ 100$ & $\$ 150$ & $\$ 300$ \\
\hline Program Benefits & $\$ 20$ & $\$ 95$ & $\$ 175$ & $\$ 300$ & $\$ 600$ \\
\hline Net Benefits & $\$ 0$ & $\$ 20$ & $\$ 75$ & $\$ 150$ & $\$ 300$ \\
\hline
\end{tabular}

In this example, Program D has the highest net benefits but exceeds the budget constraints. Using the $\$ 150$ constraint, Program $C$ provides the most benefits.

Marginal net benefit calculations are more attractive than benefit cost ratios because programs can be ranked and compared without further analysis (Beaves, Joesph, Rohrer, \& Zeitler, 1988; Birch \& Donaldson, 1987).

The marginal Benefit Cost Ratio is defined as $\mathrm{BCR}=(\mathrm{Bi}-\mathrm{Bb}) /(\mathrm{Ci}-\mathrm{Cb})$ where $\mathrm{Ci}$ $z \mathrm{Cb}$. If the $\mathrm{BCR}$ is greater than one, the new strategy is cost-effective relative to the baseline. In the case of $\mathrm{BCR}$, however, interventions cannot be compared by simply ranking their ratios. Rather, a BCR must be assessed for each pairwise comparison to determine the intervention ranking (Beaves, Joesph, Rohrer, \& Zeitler, 1988). Although two alternative programs may reveal benefit-cost ratios greater than one when compared against a baseline strategy, this does not provide information about which alternative strategy is more attractive. Consider the same programs used in the net benefit example. If the BCRs are simply ranked, Program B appears to be the most attractive program within the $\$ 150$ budget constraint. When comparing program B with program C, however, BCR equals 2.5 , revealing a more cost effective strategy. 
Table 2: An Example of Benefit Cost Ratios

\begin{tabular}{||l|c|c|c|c|c|}
\hline & Baseline & Program A & Program B & Program C & Program D \\
\hline \hline Program Costs & $\$ 20$ & $\$ 75$ & $\$ 100$ & $\$ 150$ & $\$ 300$ \\
\hline Program Benefits & $\$ 20$ & $\$ 95$ & $\$ 175$ & $\$ 300$ & $\$ 600$ \\
\hline Benefit Cost Ratio & 1.00 & 1.36 & 1.93 & 1.38 & 2.07 \\
\hline
\end{tabular}

Benefit cost ratios are also subject to measurement error when program costs and outcomes are confused (Haddix, Teutsch, Shaffer, Dunet, 1996). For example, hospital costs are taken to be a project cost when incurred but a project benefit when averted (Birch \& Donaldson, 1987). Consider the two cost-benefit strategies in which intervention benefits and costs are $\mathrm{Bi}=600$ and $\mathrm{Ci}=400$ and the baseline benefits and costs are $\mathrm{Bb}=500$ and $\mathrm{Cb}=300$. To demonstrate potential measurement error, an additional $\$ 1000$ is included as either an additional intervention benefit or intervention averted cost. Using NB calculations, $\$ 1000$ is either added as an intervention benefit or subtracted from intervention costs.

$\mathrm{NB}=(\mathrm{Bi}-\mathrm{Bb})-(\mathrm{Ci}-\mathrm{Cb})$

NB $(\$ 1000$ as Benefit $)=((600+1000)-500)-(400-300)=1000$

NB $(\$ 1000$ as Averted Costs $)=(600-500)-((400+(-1000)-300)=1000$ Although specification differences produce identical Net Benefits, they do not reveal similar BCRs.

$\mathrm{BCR}=(\mathrm{Bi}-\mathrm{Bb}) /(\mathrm{Ci}-\mathrm{Cb})$

$\mathrm{BCR}(\$ 1000$ as Benefit $)=(1600-500) /(400-300)=1100 / 100=11$

BC R $(\$ 1000$ as Averted Costs $)=(600-500) /(400-1000)-300)=100 /-900=-1 / 9$ 
BCR results are meaningless when specification errors occur.

While net-benefit analysis avoids the shortcomings of benefit cost ratios and provides more straightforward information about the allocation of resources to competing programs, it is criticized in the health field because it depends on attaching a dollar figure to health outcomes such as life years gained (Andrich, Ferrario, \& Moi, 1998; Donaldson, 1990). Although the value of life years gained can be imputed based on lost production, these valuations typically exclude quality of life issues such as changes in pain and suffering or emotional outlook (Stoddart, 1982). Moreover, imputing a dollar figure based on lost productivity (human capital approach) or willingness/ability to pay inherently favors high wage earners while it devalues low-wage earners, elderly, unemployed, students, homemakers, or volunteers (Shephard, 1989).

\section{Cost Effectiveness Analysis}

In response to these ethical dilemmas, cost-effectiveness analysis (CEA) and cost-utility analysis (CUA) strategies were developed. CEA is defined as ( $\mathrm{Hi}-\mathrm{Hb}) /(\mathrm{Fi}-\mathrm{Fb})$ where $H$ is the net health benefits and $F$ is the net financial cost. CEA measures health program outcomes in natural units such as life years gained or blood pressure lowered. Although CEA is attractive when comparing programs with similar outcomes, it does not provide information about allocating resources to programs that are measured with different health outcomes (Barry \& DeFriese, 1990; Beaves, Joesph, Rohrer, \& Zeitler, 1988). And, while cost-effectiveness ratios provide information about which program gives the greatest outcome per dollar spent, they offer no information about whether the "benefits provided per dollar are indeed worth a dollar" (Beaves, Joesph, Rohrer, \& 
Zeitler, 1988).

Like cost-effectiveness ratios, cost-utility ratios do not establish the "economic effectiveness" of different programs, i.e. CUA cannot show that the outcome is worth the dollar(s) spent. CUA does, however, provide a mechanism for comparing health interventions with different health outcomes. Cost-utility analysis reduces all health outcomes into a common utility measurement such as quality adjusted life years (QALYs) or a client satisfaction index (Hinman, 1999; Andrich, Ferrario, \& Moi, 1998). It should be noted that current recommendations from the field prescribe QALYs for all cost-effectiveness analyses so that the broad range of health care programs can be compared.

QALYs combine information about life years gained with a judgement about the quality of life in those years. Health states are adjusted for quality based on a scale from 0 (= death) to 1 (= perfect health). For instance, using QALY values from a study conducted by Fryback (1993), an additional year of life with chronic back pain is worth .74 of one year in perfect health. By utilizing quality adjusted life years, years of life with substandard health are discounted.

QALYs are the most common measure used in cost-utility analysis (Garber \& Phelps, 1997), but they have several inherent drawbacks. First, although QALY values are developed using preference ratings about defined health states, whose preferences should shape quality of life measurements? Russell, et al. (1996) state:

The choice is between patient preferences and those of a representative community sample. Patient preferences are values that people experiencing a condition assign to their own health. 
Community preferences are values assigned by representatives of the general population, which contain people with disabilities or chronic illnesses in proportion to the prevalence of the condition (p.5)

Several studies document a divergence between preference ratings of patients and the larger community (Epstein, Hall, Tognetti, Son, \& Conant, 1989; Haddix, Teutsch, Shaffer, Dunet, 1996; Murry \& Lopez, 1996). Many researchers use patient preferences because they possess greater understanding of the specific health condition and are more poised to make educated judgements about quality of life; other researchers suggest that individuals with disabilities or chronic illnesses provide overly optimistic scores about their health conditions (Russell, Gold, Siegel, Daniels, \& Weinstein, 1996).

Another drawback of the QALY is that it measures patient preferences without formally including the indirect impacts of a particular health status. For instance, a family member that provides personal care assistance free of charge is allocating resources typically excluded from the QALY outcome measurement (Andrich, Ferrario, \& Moi, 1998; Haddix, Teutsch, Shaffer, Dunet, 1996). Finally, similar to the shortcomings of the cost-benefit ratio, $\mathrm{CE}$ and $\mathrm{CU}$ ratios must be calculated for each pairwise comparison to meaningfully rank several competing interventions (Beaves, Joesph, Rohrer, \& Zeitler, 1988).

Given the drawbacks inherent in each evaluation strategy, it is not surprising that conflicting recommendations arise from the health field about which analysis to pursue. Drummond et al. (1987) and Birch \& Donaldson (1987) support the use of net-benefit analysis as the most comprehensive strategy. Beaves, et al. (1988) also defend the use of 
net benefit analysis rather then cost-benefit or cost-effectiveness ratios, but attempts to quantify QALY estimates for the benefit measurement.

Despite recommendations for a net-benefit analysis, CEA is attractive when comparing programs that have a common effect (Haddix, Teutsch, Shaffer, Dunet, 1996; Russell, Gold, Siegel, Daniels, \& Weinstein, 1996). Once efficacy and efficiency have been determined, CEA can avoid the difficulties associated with quantifying health outcomes. Moreover, for interventions which do not impact extended life years or quality of life years such as programs to reduce the number of dental cavaties, CEA can provide a straightforward comparison. The relative ease of calculating $\mathrm{CE}$ ratios makes it a popular choice in the field (Messonnier, Corso, Teutsch, Haddix, \& Harris, 1999) and the subject of several economic discussions (Russell, Gold, Siegel, Daniels, \& Weinstein, 1996).

Despite conflicting views about methods of program analysis, there is solid agreement in the literature concerning analytical perspective. From the standpoint of rigor and comparability across studies, panel members convened by the US Public Health Service suggest a societal perspective. "While the use of a particular perspective (eg, HMO, employer, government program, or individual) is appropriate for informing decisions from that perspective, studies based on different perspectives are not comparable...To serve the goal of facilitating comparisons across interventions, the panel recommends the societal perspective for the reference case" (Russell, Gold, Siegel, Daniels, \& Weinstein, 1996, p.3).

The societal perspective provides information about a program's social value without confining costs and benefits to the financial outlays of one particular individual 
or group (Andrich, Ferrario, \& Moi, 1998). Consider an intervention that allows movement from a hospital setting to a patient's home. If a hospital perspective were adopted, indirect costs such as time provided by family members to bridge this transition. might be excluded from the cost-benefit calculations. Haddix et al. (1996) also point out that health strategies can have outcomes that are much broader than the particular health conditions they are designed to impact. Consider the case of smoking cessation programs which provide benefits to society through reduced second hand smoke and better driving records. Obviously, individual perspectives have the potential to misinform overall resource-allocation decisions (Haddix, Teutsch, Shaffer, Dunet, 1996).

Despite agreement about the desirability of a societal perspective, Messonier, et al. (1999) acknowledge that "including all costs and benefits can be difficult, and thus, there is not strict adherence to the recommended societal perspective" (p.261). Moreover, it is recognized that individual perspectives are necessary to inform some decisions. Members of the US Public Health Service panel (1996) state that the societal perspective does not show individuals or groups the information they need to make choices suited to their own interests (Russell, Gold, Siegel, Daniels, \& Weinstein, 1996).

Finally, professional recommendations pertaining to health care evaluation also include suggestions about the analytic horizon. Analytic horizon is the period over which the costs and benefits of a particular health intervention are considered (Haddix, Teutsch, Shaffer, Dunet, 1996). In general, the analytic horizon needs to be long enough to capture intervention outcomes and avoid seasonal impacts, while simultaneously minimizing the probability of unexpected life events (Andrich, Ferrario, \& Moi, 1998). 
The analytic horizon is particularly problematic for health promotion/disease prevention programs since health outcomes can be far-reaching and clouded by uncertain causal relationships. It is difficult to predict future environmental factors, to measure long-term health outcomes, and to measure adherence to targeted health behaviors (Thacker, et al., 1994).

Literature about the economic evaluation of health care programs is rich, but because of the high degree of complexity involved, consensus on a particular strategy is lacking (Haddix, Teutsch, Shaffer, Dunet, 1996). In the face of scarce public health resources coupled with exponentially increasing health care costs, the effectiveness and cost of new health interventions, strategies, and technologies are subject to more scrutiny than ever before. In the wake of this trend, professionals continue to develop and refine strategies to allocate scarce health resources both ethically and efficaciously.

\section{Current Studies of Health Promotion/Disease Prevention Interventions}

\section{for Individuals with Disabilities}

Evaluations of health promotion/disease prevention programs for individuals with disabilities are scarce (Rimmer, 1999), and of the few studies that appear in the literature, most are exploratory and answer questions about efficacy rather than costs. These efficacy studies target individuals with specific chronic conditions including multiple sclerosis (Husted, Pham, Hekking, \& Niederman, 1999), spinal cord injury (Edwards, 1996), mobility impairments (Kinne, Patrick, \& Maher, 1999; Maher, Kinne, \& Patrick, 1999) obstructive pulmonary disease, chronic low-back pain, cystic fibrosis, osteoarthritis (Taylor, Baranowski, \& Young, 1998), and arthritis (Lorig, Gonzales, Laurent, \& Laris, 
1998; Lorig, Gonzalez, \& Ritter, 1999; Lorig, et al., 1999). Although these studies do not inform further research about cost-benefit analysis, they offer some insight into experimental design.

Lorig and associates provide several studies about the Arthritis Self-Management Program (ASMP), which has been supported by the national Arthritis Foundation since the mid-1980s. To assess efficacy of different versions of the ASMP, Lorig et al. (1998), collected self reported data on 151 subjects who were assigned to either a 6 week or 3 week ASMP intervention. Lorig et al. compared 11 health measures immediately before (baseline) and 4 months after program participation with a 2-tailed paired t-test for significance. While both the 3 week and 6 week groups had significant improvements in health distress, cognitive pain management, and self-efficacy (personal ability to affect results), the six week course resulted in additional improvements including pain reduction, illness impact, exercise, and reduced visits to the physician. Despite higher implementation costs, these results showed better health outcomes for the 6 week ASMP intervention.

In a later study by Lorig et al. (1999), the ASMP program was evaluated in a community setting for Spanish speaking participants. Program participants completed self report surveys at baseline, 4 months, and 1 year. Although all recruited subjects participated in the ASMP, 1 out of every 3 participants was placed on a 4 month waiting list and served as an extended baseline control group. Controlling for age, gender, education, non-steroidal anti-inflammatory drug use, and baseline self-efficacy, the study and extended baseline control groups were compared at 4 months using an analysis of 
covariance (ANCOVA) for nine outcome variables. Significant differences between the extended baseline and treatment groups included a reported $16 \%$ reduction in pain and $11 \%$ decrease in disability for the treatment group as compared to no change in the extended baseline group.

A 1-year longitudinal study was also conducted using paired $t$ tests between baseline and 1 year data; baseline and 4 months data; and 4 months and 1 year data. Results between baseline and 1 year data showed significant changes in seven outcome measures including range of motion, aerobic exercises, self-efficacy, disability, pain, depression, and self-rated health status. Baseline to 4 months data also had seven significant outcome measures (disability was non-significant but outpatient doctors visits was significant). Health outcomes were non-significant for all measures between 4 months and 1 year. In general, gains made during the first four months of the SASMP program were maintained for 1 year.

Leveille et al. (1998) evaluated a community based health promotion intervention, the Health Enhancement Project (HEP), for the elderly frail. Self report surveys were completed for both control and participant subjects at baseline, 6 months, and 12 months after baseline. Health utilization information was collected from administrative databases maintained by HMOs. Outpatient utilization included "primary and specialty care visits, home health visits, mental health and emergency room visits, and ancillary outpatient services excluding radiology and laboratory services" (Leveille, et al., 1998, p.1193). Baseline differences between the study and control groups were tested with $t$ tests for continuous health outcome variables and chi-square tests for categorical health outcome 
variables. To control for baseline differences between groups, subjects were categorized into high function vs. limited function groups based on distributions of bed days and restricted activity days. Intermediate health outcomes were compared using adjusted least square means averaged over 6 and 12 month follow-ups. Although health utilization declined significantly for the study group, there were no costs attached to health utilization outcomes.

In another study sponsored by the Arthritis Foundation, Kruger, et al. (1998) analyzed the cost-effectiveness of the Arthritis Self-Management Course (ASMC) from both the societal and health care system perspectives. The ASHC course was implemented in a community setting across 6 weeks in 2 hour weekly segments. Extended baseline subjects were placed on a 4 month wait list before receiving the ASCH intervention. Self-report data were gathered for all intervention subjects at baseline, 4 months, and 4 years following the intervention. Extended baseline subjects had an additional questionnaire at 4 months pre-intervention. There was no formal control group for the $\mathbf{4}$ year follow-up, but health outcomes were compared to similar groups with osteoarthritis and rheumatoid arthritis.

Program participants provided data about the number of times they saw a physician for arthritis-related symptoms, time to travel and attend arthritis-related doctor appointments or the ASHC workshop, and several health measures. The societal perspective included program costs per enrollee, costs associated with arthritis related physician visits, and personal costs including time and transportation for attending the ASHC and arthritis-related physician visits. For the perspective of the health care 
system, costs included only ASHC program costs per enrollee and costs for arthritisrelated physician visits.

The unit cost per physician visit was estimated at $\$ 50$ per visit and was taken from the Physician's Fee and Coding Guide. The cost of personal time was estimated at $\$ 8.70$ per hour, the average hourly rate for the mean age of program participants. Travel costs were calculated at .25 per mile. Costs to implement the ASHC workshop were estimated at $\$ 76$ per participant based on past cost experiences at the Arthritis Foundation to implement the ASCH program. From past studies conducted by Lorig and others, Krugar projected that the average number of arthritis-related physician visits would be 3 times per year for individuals who completed the ASHC workshop and that 90 percent of participants who began the program would complete.

The authors estimated that 4 years post intervention, the ASHC program produced cost savings from both the societal and health care system perspectives. Using the societal perspective, the ASHC program reduced pain by .9 units $(18 \%)$ at a savings of $\$ 320$; from the health care system perspective pain reduced by .9 units (18\%) at a saving of \$267. To demonstrate the strength of his results, Kruger et al. used univariate analyses on key cost variables to determine threshold values where the program would become cost incurring rather than cost savings. In contrast to projected participation rates and unit costs, threshold values for the societal perspective include: cost to participate in the ASHC program per participant (\$398); cost for an arthritis related physician visit (\$13.41); number of arthritis related physician visits per year for ASCH completers (4.5), and probability of completing the ASHC (.20). Even using multivariate analysis to 
construct a worst case scenario with threshold values occuring simultaneously, the societal perspective resulted in a small net cost of $\$ 198$ over 4 years to reduce pain by .32 units.

Lorig et al. (1999) addressed questions of intervention costs and benefits in a study of the Chronic Disease Self-Management Program (CDSMP). The CDSMP is particularly interesting because it closely parallels the implementation of Living Well with $a$ Disability. Like Living Well, the CDSMP is a community based patient education course, delivered by a pair of lay instructors/facilitators to small groups with mixed ages and chronic conditions, in weekly 2.5 hour segments. CDSMP subject matter is similar to Living Well and addresses a broad range of health issues including problem-solving, decision-making, depression, nutrition, and symptom management.

Data were collected for CDSMP program participants at baseline and at six months. Control subjects were invited to participate in the CDSMP program after 6 months on a waiting list. Subjects were randomized at a rate of 6 study participants to 4 extended baseline subjects. Participants completed mailed questionnaires that addressed questions about health behaviors, health status, and health service utilization. Health service utilization questions asked about the number of physician and emergency room visits, and hospital nights during the preceeding 6 months. Utilization data was crossreferenced against automated HMO medical records.

When program costs and outcomes were examined, the decrease in hospital nights was significant between the study and extended baseline groups. This significance was not extended to changes in doctor and emergency room visits. Using an estimate of 
$\$ 1,000$ per hospital day, the extended baseline group spent approximately $\$ 820$ more per subject than the treatment group. Program costs were estimated at $\$ 70$ per participant for a net gain of $\$ 750$ per participant. It should be noted that program costs did not include any indirect costs or costs for space to conduct the intervention. Additionally, the authors did not perform any sensitivity analyses to test the robustness of these results including variations in hospital costs or number of participants in each intervention session.

Cronan et al. (1998) conducted a cost-benefit ratio analysis on three interventions aimed at osteoarthritis. Interventions include a social support intervention, an education intervention, and a combined social support and education intervention. The three study groups and a control group each had between 80 and 100 participants recruited from an HMO. Health care utilization rates were secured through participant medical records and included data about "number of physician contacts, urgent care contacts, contacts with nurses, nurse practitioners, physician's assistants, hospital visits, emergency room visits, days in the hospital, and home visits" (Cronan, et al., 1998, p.326). Data was collected at baseline, and at 1,2, and 3 years. Benefits were defined as the change in costs from baseline to 1-, 2-, and 3-years post intervention. These health care costs were calculated from national averages provided by the US Health Care Financing Administration.

Benefits (cost savings) were discounted at $5 \%$ per year. Health care status improved for all intervention groups, but the cost-benefit ratio was lowest for the social support group.

Initially, the three intervention groups were compared using repeated measures analysis of variance (ANOVAs) to determine if the groups differed in changes in health care costs between the baseline, 1, 2, and 3 year assessments. Since no group effect or 
interaction was found to be significant, the three intervention groups were collapsed into one experimental group for further comparisons with the control group. Again, ANOVA was used to determine differences in changes in health care costs at each assessment point for the control and experimental group. In this case, there were significant differences between the control and experimental groups across time in terms of health care costs. The first, second and third year benefit cost ratios are 14.2, 27.2 and 30.0 when the three experimental group costs and benefits were combined. Sensitivity analyses were performed for different cost (savings) scenarios including higher hospital rates for the first three days (to more accurately reflect initial hospital days in which most diagnostic tests and surgical procedures tend to occur).

\section{Current Studies of Health Promotion/Disease Prevention Interventions}

\section{for Individuals without Disabilities}

Cost benefit literature is broader for health promotion programs directed at the general population. Particularly in terms of work-site health promotion/disease prevention activities, there are several cost-benefit and cost-effectiveness studies to choose from. One advantage of work-site health promotion studies is the availability of medical claims data through employer sponsored insurance carriers. It should be noted that the majority of work-site studies are analyzed from the perspective of the employer or insurance carrier, rather than from a societal perspective.

Aldana et al. (1993) performed a financial cost-benefit analysis of the CIGNA Healthplan Worksite Wellness Program. While this analysis had a true control group it was not a randomized design; study participants volunteered to take part in the CIGNA 
Program while individuals in the control group did not. Medical claims data from the Mesa insurance plan was provided over a four year period ( 2 years pre-intervention and 2 years post-intervention) for study participants and control subjects. Because wide variances in medical claims data resulted in an abnormal distribution, log base transformations were performed on the dollar cost figures. The authors used these transformed cost figures in a " $2 \times 2$ repeated measure analysis of variance" (MANOVA) to determine if the change in costs were significantly different between preprogram and postprogram claims data. MANOVA differs from ANOVA because it can measure several dependent variables in a single statistical equation by creating artificial dependent variables (French \& Poulsen, 1999). The authors found a significant difference between the participant and control groups at pre-intervention where averaged pre-program costs over six months was $\$ 2,148$ per study participant and $\$ 1,480$ per control subject. Additionally, averaged pre-program (four time periods) and post-program (four time periods) claims data showed a $16 \%$ decrease in health care utilization costs for study participants and a $7 \%$ decrease in health care utilization costs for the control group. This unexpected decrease in costs for the control group could have been due to social interactions between study participants and non-participants or from reaction to the testing procedures (Aldana, Jacobson, Harris, Kelley, \& Stone, 1993).

A benefit cost ratio of the CIGNA program combined claims data for both the study and control groups. The benefit-to-cost ratio of 3.6 was calculated using benefits (defined as the decrease in health care costs between pre-intervention and postintervention claims data) divided by total program costs. The study and control groups 
were combined for this benefit-to-cost ratio since programmatic costs of work-site programs are borne by the entire corporation workforce, regardless of participation. It is important to note that calculation of program benefits ignored several additional program outcomes, including changes in productivity, workplace absenteeism, and pension costs.

In another randomized controlled health promotion study, Fries, et al. (1994) estimated reductions in health care costs from self reported data about doctor visits, hospital days, and days confined to home to evaluate two health promotion interventions targeted at three specific groups: employees, retirees, and seniors. Blue Cross/Blue Shield medical claims data complimented self report data for the employee group. Due to wide variance in medical claims and self report data, log linear transformations were performed on health utilization data before groups were compared using t-tests for significance. Results indicated that overall claims decreased by approximately $\$ 148$ per person per year across the three intervention groups.

Sciacca, et al. (1993) also found wide variances and skewed distributions for medical claims data collected for the Blue Cross and Blue Shield of Indiana (BCBSI) Health Promotion Service. The BCBSI study employed two treatment and two control groups with data collected from BC/BS medical claims two years prior and five years post intervention. Instead of transforming the cost variables using a log transformation, the Jonckheere-Terpesta non-parametric test statistic was used to analyze pre and post data on health care costs. "The Jonckheere-Terpesta test statistic is based on the total number of times pre to post increases in health costs are greater among subjects in one group than among those in other groups" (Sciacca, Seehafer, Reed, \& Mulvaney, 1993, 
p378).

Contrary to other analyses, this study did not reveal any statistically significant cost reductions of the BCBSI health promotion program. Sciacca defends his results because the baseline (collected across 2 years pre-intervention) and study period (collected across 5 years post-intervention) were sufficiently large to more accurately reflect average health care utilization and the use of non-parametric statistics provides a mechanism in which the entire study sample could be used without removing outliers.

Shi (1993) evaluated different levels of work-site health promotion interventions using marginal net benefits. A control group and three intervention groups with increasing health promotion intensities were compared. Shi defined costs as payments to outside consultants and providers who designed and implemented the program, program materials, and wages for staff involved in the program. Benefits include cost savings due to changes in medical utilization and changes in number of reported sick days. Shi calculated both net benefits and return on investment defined as (net benefits) divided by (total costs).

ANOVAs were used to evaluate between group differences at baseline for outcome variables including hospital days, doctor visits, and sick days. To adjust for non-equivalent groups, repeated measures analysis of covariance (ANCOVA) was used to assess net benefits during the study period for each intervention group. ANCOVA controls for variation among the covariate(s) and consequently reduces the error variance and bias caused by differences between experimental groups (Kirk, 1995). Shi used a least square means procedure (two-tailed tests for significance) to compare group means 
for all significant ANCOVA effects. The net benefits for the four intervention strategies ranged from $\$ 87$ to $\$ 248$ per person.

In addition to the methodology reported in the previous health promotion studies, several articles address research questions which are pertinent to Living Well with a Disability. In a cost-effectiveness study of a primary care physical activity intervention, Stevens, et al. (1998) found that the most significant factor affecting cost-effectiveness was recruitment success. Although the physical activity intervention was geared to serve 363 attendees, unsuccessful recruitment resulted in a $35 \%$ response rate and, subsequently, underused resources. A sensitivity analysis of recruitment up-take revealed that unit costs could have been halved if the maximum number of participants had been treated. Recruitment levels are an important consideration of the Living Well intervention, which was designed to serve up to 12 participants per workshop, but typically served fewer.

Using hierarchical multiple regression, Golaszewski, et al. (1989) studied the efficacy of health risk appraisal to predict medical care utilization rates. Using medical claims data as the dependent variable and age and health index as the independent variables, health index scores were found to be predictive for male health utilization rates. Health index scores are similar to the sum of secondary condition scores reported in the Living Well data and provide an intermediate outcome measure for cost-effectiveness. Golaszewski, et al. (1989) also found that several variables had significant relationships to health care utilization rates including age, sex, geographic region, socioeconomic status, type of insurance coverage and personal lifestyle. 
Another consideration when examining Living Well involves the impact of changing age on cohort medical utilization rates. Bowne, et al. (1983) ignores age factors for changes of one year or less. Fries, et al. (1994) also does not age-adjust his employed study group since the self-reported health costs below age 55 have relatively small changes in one year increments. However, for populations over age 55, baseline data from Fries, et al. showed a .1 day increase in hospital use per year over age 55. Approximately $25 \%$ of Living Well participants are over age 55 .

Finally, it will also be important to address several measurement errors when analyzing the self-report survey collection methods of Living Well. First, medical care utilization tends to be under-reported or miss-reported, as evidenced by studies which compare self-report with actual medical claims data (Lorig, Gonzales, Laurent, \& Laris, 1998; Lorig, et al., 1999). Second, the Hawthorne effect potentially over-states health limitations (in baseline data) and health improvements (in post intervention data). The Hawthorne effect occurs when individuals who know they are being studied answer questions based on how they expect the intervention to work rather than reporting actual program impacts (Shi, 1993; Bertera, 1993). Third, there is evidence that just being questioned about certain health activities may result in subtle health behavior changes. Shi (1993) found that his control sample of 412 individuals reported significant reductions in risk behaviors between pre and post questionnaires. Control subjects may have been motivated by health risk assessment questions to change certain risk behaviors. Analysis of the Living Well with a Disability intervention will draw on many of the recommendations, questions, and concerns addressed in the health promotion/disease 
prevention literature. Using experience from the field, the task at hand is to provide the most comprehensive analysis of Living Well within the constraints of the data collected. 


\section{Chapter 3: Methods and Analysis}

The Living Well with a Disability Program is the outcome of ten years of research at the University of Montana about the incidence and severity of secondary conditions for people with physical impairments. Secondary conditions refer to a range of health complications that occur in conjunction with a primary disability. For instance, a person with a spinal cord injury (primary disability) is at greater risk of pressure sores and urinary tract infections (secondary conditions) because of sensation loss. Although some secondary conditions are difficult to manage, there are several health conditions that can be affected by changes in lifestyle.

\section{Living Well Workshops}

The Living Well workshop uses goal setting and problem solving to manage health outcomes for people with physical impairments. Making the connection between health and function is the foundation of the Living Well program. For instance, a person might work on flexibility and strength so she can independently ride the bus to a friend or family member's house. Rather than encourage exercise and strength training to achieve better health, participants are asked to set goals, like visiting grandchildren or gaining employment, as the impetus to improve strength. Health is portrayed as a means to a goal, rather than the goal itself.

The Living Well program is delivered in eight weekly two hour sessions by two lay facilitators. Each session includes lecture, in-class exercises, and group sharing. Specific content areas include goal setting, problem solving, healthy reactions, combating depression, healthy communication, seeking information, physical activity, nutrition, 
access issues, and health maintenance.

\section{Independent Living Centers}

Independent Living Centers (ILCs) were selected as the best venue to deliver the Living Well workshop to individuals with mobility impairments. During November of 1997, 336 ILCs and 245 ILC satellite offices throughout the United States were sent applications to participate in the study. As part of the application process, ILCs were asked to respond to a list of competencies including demonstrated ability to provide workshop training, accessible space, willingness and ability to advertise the workshop, ability to collect data, familiarity with health promotion, and staff capacity. One hundred and six (106) ILCs completed and returned applications to the University of Montana. A national advisory group selected nine ILCs to participate in the program. Selected ILCs came from both rural and urban regions, southern and northern tier states, and from states with high and low per capita income and education levels (U.S. Census Bureau, 1999). Selected ILCs received facilitator training, Living Well workbooks, and $\$ 2,430$ to cover staff salary, meeting space, refreshments, and other incidentals for each replication of the Living Well workshop.

Thirty-four (34) rounds of Living Well workshops were conducted over two years. Workshops were conducted by teams of two facilitators (two ILC staff, or one ILC staff and one consumer) from each ILC. The following table provides the intervention dates and ILCs involved. Workshop replications were delivered at four different times throughout the year to offset potential seasonal and economic effects. 
Table 3: Living Well Implementation Dates

\begin{tabular}{|l|l|l|l|l|l|l|l|l|l||}
\hline \multicolumn{1}{|c|}{ ILC } & $4 / 98$ & $6 / 98$ & $8 / 98$ & $10 / 98$ & $4 / 99$ & $6 / 99$ & $8 / 99$ & $10 / 99$ & Total \\
\hline $\begin{array}{l}\text { Hays, Kansas } \\
\text { LINK }\end{array}$ & $\mathrm{X}$ & $\mathrm{X}$ & & & $\mathrm{X}$ & $\mathrm{X}$ & & & 4 \\
\hline $\begin{array}{l}\text { Austin, Texas } \\
\text { ARCIL, Inc. }\end{array}$ & $\mathrm{X}$ & $\mathrm{X}$ & & & $\mathrm{X}$ & $\mathrm{X}$ & & & 4 \\
\hline $\begin{array}{l}\text { Springfield, Missouri } \\
\text { Southwest Center for IL }\end{array}$ & $\mathrm{X}$ & $\mathrm{X}$ & & & $\mathrm{X}$ & $\mathrm{X}$ & & & 4 \\
\hline $\begin{array}{l}\text { Black Eagle, Montana } \\
\text { North Central IL Services, Inc. }\end{array}$ & $\mathrm{X}$ & $\mathrm{X}$ & & & $\mathrm{X}$ & $\mathrm{X}$ & & & 4 \\
\hline $\begin{array}{l}\text { Jackson, Mississippi } \\
\text { LI in Central MS }\end{array}$ & $\mathrm{X}$ & $\mathrm{X}$ & & & $\mathrm{X}$ & $\mathrm{X}$ & & & 4 \\
\hline $\begin{array}{l}\text { Ithaca, New York } \\
\text { Finger Lakes ILC }\end{array}$ & & & & & $\mathrm{X}$ & & & $\mathrm{X}$ & 2 \\
\hline $\begin{array}{l}\text { Anaheim, Califormia } \\
\text { Dayle McIntosh Center }\end{array}$ & & $\mathrm{X}$ & $\mathrm{X}$ & & & $\mathrm{X}$ & $\mathrm{X}$ & 4 \\
\hline $\begin{array}{l}\text { Columbia, Missouri } \\
\text { LC of Mid MO dba }\end{array}$ & & & $\mathrm{X}$ & $\mathrm{X}$ & & & $\mathrm{X}$ & $\mathrm{X}$ & 4 \\
\hline $\begin{array}{l}\text { Concord, New Hampshire } \\
\text { Granite State IL Foundation }\end{array}$ & & & $\mathrm{X}$ & $\mathrm{X}$ & & & $\mathrm{X}$ & $\mathrm{X}$ & 4 \\
\hline $\begin{array}{l}\text { Total Living Well } \\
\text { Replications }\end{array}$ & 5 & 5 & 3 & 3 & 6 & 5 & 3 & 4 & 34 \\
\hline
\end{tabular}

\section{Participant Recruitment}

Living Well facilitators were responsible for participant recruitment. Participant recruitment efforts were conducted once per year for each intervention site. Recruitment efforts included letters to consumers, personal communications between the facilitators and ILC consumers, posted flyers, media (news releases, interviews with the media, Public Service announcements), and referrals from medical service providers who received information packets about the Living Well workshops. Living Well group 
facilitators tracked the number of contacts (letters sent, individuals called, flyers posted, etc.) for each recruitment strategy. Table 4 shows the average contact rates for each Living Well recruitment method and the average number of consumers who responded to each across all intervention sites. For each round of recruitment, participants were randomly assigned to one of two intervention groups. The first group began immediately and the second group began two months after recruitment and served as an extended baseline. (The Finger Lakes Independent Living Center did not have an extended baseline group and recruited for each Living Well implementation.)

Table 4: Living Well Recruitment Methods

\begin{tabular}{|c|c|c|c|c|c|c|}
\hline Advertisement Strategy & $\begin{array}{l}\text { Recruitment } \\
\text { Contacts - } \\
\text { 1st Round } \\
\text { Mean \# }\end{array}$ & $\begin{array}{c}\text { Participant } \\
\text { Inquiries - } \\
\text { 1st Round } \\
\text { Mean \# }\end{array}$ & $\%$ & $\begin{array}{c}\text { Recruitment } \\
\text { Contact - } \\
2^{\infty} \text { Round } \\
\text { Mean \# }\end{array}$ & $\begin{array}{c}\text { Participant } \\
\text { Inquiries - } \\
2^{\text {ma }} \text { Round } \\
\text { Mean \# }\end{array}$ & $\%$ \\
\hline $\begin{array}{l}\text { Letters sent to ILC } \\
\text { members }\end{array}$ & 117 & 11.8 & $10.1 \%$ & 84 & 8.3 & $9.9 \%$ \\
\hline Flyers posted & 150 & 3 & $2 \%$ & 214 & .25 & $.1 \%$ \\
\hline $\begin{array}{l}\text { Personal communications } \\
\text { (between workshop } \\
\text { facilitators and consumers) }\end{array}$ & 31 & 7.17 & $23.1 \%$ & 39 & 6.75 & $17.3 \%$ \\
\hline $\begin{array}{l}\text { Media communications } \\
\text { (number of interviews, } \\
\text { PSAs, and news releases) }\end{array}$ & 68 & 4.2 & $6.2 \%$ & 54 & 2.75 & $5.1 \%$ \\
\hline $\begin{array}{l}\text { Information packets sent to } \\
\text { medical personnel }\end{array}$ & 79 & .5 & $.6 \%$ & 84 & 0 & $0 \%$ \\
\hline
\end{tabular}

The most successful forms of advertising were letters and personal communications between ILC staff and ILC client members. The average number of participants recruited for the first round of recruitment was 8.3 participants per Living Well workshop whereas the second round of recruitment had an average rate of 5.6 participants per Living Well workshop. 
A total of 246 participants were recruited to participate in the Living Well workshops. Small and declining recruitment levels were a problem since the Living Well workshop is designed to serve up to 12 participants. Potential reasons for poor recruitment rates are a small population with physical impairments to draw from and ineffective marketing strategies. Since more enthusiastic participants were probably recruited in the first round, it is not surprising that recruitment for the second round of recruitment may have been less effective.

\section{$\underline{\text { Data Collection }}$}

Participant data were collected via a participant survey at five points in time (survey B -immediate pre-intervention, survey C - immediate post-intervention, survey D - 2 months post-intervention, survey $\mathrm{E}$ - 4 months post-intervention, and survey $\mathrm{F}$ - one year post intervention). An additional wave of data was collected for approximately half of the participants who were randomly assigned to wait two months before receiving the Living Well intervention. This additional data wave, survey A, was collected two months prior to intervention and served as an extended baseline measure.

A sample survey is attached in Appendix A. Briefly, the data collected included:

(1) Demographics: Age, gender, rural/urban location, education level, marital status, race, ethnicity, employment status, health care coverage, and disability information.

(2) Secondary Conditions: The Surveillance Instrument of Secondary Conditions (SCSI) measures the time limitation that individuals experience due to specific secondary conditions (Ravesloot, Seekins, \& Quincy-Robyn, 1998). Respondents 
are asked to rate each secondary condition on a scale from 0 to 3 . A zero (0) rating indicates rare limitation or non-experience of the listed secondary condition; a one (1) response indicates mild or infrequent limitation ( 1 to 5 hours per week); a two (2) response indicates a moderate limitation (6-10 hours per week); and a three (3) indicates a significant/chronic limitation (more than 11 hours per week).

(3) Health Care Utilization Measures: Utilization data includes information about hospital, emergency room, outpatient, and doctors visits; test, services, and treatments; medications; personal assistance needs; and cost of medical care and income data.

(4) Health Status: Twenty-one (21) health status questions were taken from the Behavioral Risk Factor Surveillance System (BRFSS). The BRFSS was developed as part of the U.S. Department of Health State Based Capacity Building Grants. Since 1980, the BRFSS questionnaire has been actively used in all 50 United States. Including BRFSS health status questions provides a comparison measure between the study sample and the general population.

(5) Depression: Depression was measured with the Centers for Epidemiology Study of Depression Scale (Radloff, 1977). This scale poses 20 questions about an individual's current depressive symptoms, with an emphasis on depressed mood (Ravesloot, Seekins, \& Quincy-Robyn, 1998). Respondents are asked to rate the frequency with which statements occur on a scale of 1 to 4 where 1 indicates a rare occurrence (less than 1 day per week) and 4 is something that occurs most of 
the time (5-7 days per week).

(6) Lifestyle: The Health Promoting Lifestyle Profile has 48 questions about different lifestyle characteristics including self-actualization, health responsibility, exercise, nutrition, interpersonal support, and stress management (Walker, Sechrist, \& Pender, 1987). Respondents are asked to rate responses on a Likert type scale where $\mathrm{N}=$ never, $\mathrm{S}=$ sometimes, $\mathrm{O}=$ often, and $\mathrm{R}=$ routinely.

(7) Quality of Life: Four questions focus on quality of life measures, including physical suffering, outlook, daily activities, and overall life quality. These measures were drawn from Hadorn and Uebersax's Quality of Life and Health Questionnaire (Hadorn \& Uebersax, 1995).

(8) Barriers: The 28 questions relating to barriers were adopted from the Barriers to Health-Promoting Activities for Disabled Persons Scale (Becker, Stuifbergen, \& Sands, 1991). These questions examine possible barriers to participation in the Living Well and other health related programs, such as pain, fatigue, accessibility, transportation, and cost.

Measures one through seven were repeated at each data collection point to track health status before and after the Living Well intervention. The additional set of questions concerning barriers (measure 8) was included at the A (2-months pre-intervention), B (immediate pre-intervention), and C (immediate post-intervention) data collection points.

Finally, data collection at points C (immediate post-intervention), D (2-month post-intervention), E (4-month post-intervention), and $\mathrm{F}$ (1-year post-intervention) contained questions relating to Living Well workshop content regarding goal-setting. 


\section{Cohort Description}

Although 246 participants were recruited into the Living Well Program, only 188 participants completed both the B (immediate pre-intervention) and C (immediate postintervention) data collection instrument. Because this study relies on comparing health care utilization across time, this analysis is confined to individuals who provided both pre-and post-intervention data. Table 5 describes the 188 study participants.

\section{Table 5: Participant Demographics}

\begin{tabular}{|c|c|c|c|}
\hline Demographics & Mean & $\begin{array}{l}\text { Std. } \\
\text { Dev. }\end{array}$ & Valid Percent \\
\hline Age at intervention $(n=187)$ & 45.39 & 13.42 & \\
\hline Years with disability $(n=151)$ & 17.48 & 15.65 & \\
\hline $\begin{array}{c}\text { Location }(n=187) \\
\text { Rural } \\
\text { Urban }\end{array}$ & & & $\begin{array}{l}31.0 \% \\
69.0 \%\end{array}$ \\
\hline $\begin{array}{c}\text { Gender }(n=187) \\
\text { Male } \\
\text { Female }\end{array}$ & & & $\begin{array}{l}35.8 \% \\
64.2 \%\end{array}$ \\
\hline $\begin{array}{l}\text { Disability Type }(n=183) \\
\text { Stable } \\
\text { Degenerative }\end{array}$ & & & $\begin{array}{l}48.1 \% \\
51.9 \%\end{array}$ \\
\hline Years of Education $(n=179)$ & 13.66 & 3.32 & \\
\hline $\begin{array}{c}\text { Marital Status }(\mathrm{n}=186) \\
\text { Not married } \\
\text { Married }\end{array}$ & & & $\begin{array}{l}63.4 \% \\
36.6 \%\end{array}$ \\
\hline $\begin{array}{l}\text { Race }(\mathrm{n}=188) \\
\text { White } \\
\text { Black/African American } \\
\text { Hawaiian/Pacific Islander } \\
\text { Asian } \\
\text { American Indian/Alaska Native }\end{array}$ & & & $\begin{array}{c}82.4 \% \\
13.8 \% \\
.5 \% \\
.5 \% \\
2.7 \%\end{array}$ \\
\hline
\end{tabular}




\begin{tabular}{|c|c|c|c|}
\hline Demographics & Mean & $\begin{array}{l}\text { Std. } \\
\text { Dev. }\end{array}$ & Valid Percent \\
\hline $\begin{array}{l}\text { Ethnicity }(\mathrm{n}=188) \\
\text { Hispanic or Latino } \\
\text { Other }\end{array}$ & & & $\begin{array}{c}3.2 \% \\
96.8 \%\end{array}$ \\
\hline $\begin{array}{l}\text { Employment Status }(\mathrm{n}=185) \\
\text { Employed (part or full time) } \\
\text { Not employed }\end{array}$ & & & $\begin{array}{l}16.2 \% \\
83.8 \%\end{array}$ \\
\hline $\begin{array}{l}\text { Insurance Status ( } \mathrm{n}=184 \text { ) } \\
\text { Medicaid only } \\
\text { Medicare only } \\
\text { Private only } \\
\text { VA only } \\
\text { Medicaid/Medicare } \\
\text { Medicaid/VA } \\
\text { Medicaid/Private } \\
\text { Medicaid/Medicare/VA } \\
\text { Medicaid/Medicare/Private } \\
\text { Medicare/VA } \\
\text { Medicare/Private } \\
\text { Medicare/VA/Private } \\
\text { No Insurance }\end{array}$ & & & $\begin{array}{c}23.4 \% \\
12.5 \% \\
15.2 \% \\
1.0 \% \\
22.3 \% \\
0 \% \\
4.3 \% \\
1.0 \% \\
4.9 \% \\
.5 \% \\
9.2 \% \\
2.2 \% \\
\\
3.3 \%\end{array}$ \\
\hline
\end{tabular}

Age and years with a disability were referenced to the time of intervention, 1998 and 1999. Rural and urban locations were calculated using the county designations as defined by the US Census Bureau (1999). The stable versus degenerative disability categories classify impairments as typically static over time or impairments that have degenerative features. (For instance, a spinal cord injury would be considered stable, and multiple sclerosis would be categorized as degenerative - severity increases over time). 
Marital status was either not married (never married, divorced, or widower) and married (including separated and significant relationship). Employed referred to individuals working full or part time and not employed included individuals who reported being a retiree, homemaker, student, volunteer or not currently employed. The study participants had one (or more) of four types of insurance including Medicaid, Medicare, private insurance, and VA coverage. Percentages are given for the number of individuals covered under each insurance type or combination of insurance types.

\section{Cost-Benefit Measures}

Cost Benefit calculations are based on data collected from the 188 participants (described above) who completed both a B (immediate pre-intervention) and C (immediate post-intervention) survey. Although this data sample excludes individuals who may have completed (and benefitted from) part of the Living Well workshop series, it provides a clear benchmark for the study sample.

\section{Living Well Implementation Costs}

Programmatic costs include fixed costs for contracted services to implement the Living Well workshop and instructor training, and variable costs for participant workshop materials. Each round of the Living Well with a Disability workshop was contracted for $\$ 2,430$. This amount was meant to cover all expenses associated with the workshop including accessible space to hold the workshop, recruitment efforts, training costs, payment for lay instructors, and other incidentals such as interpreter services or readers.

Facilitator training costs came to $\$ 26,528$ across the two years of the project. A breakdown of training costs for 1998 and 1999 is included in Appendix B. Workshop 
materials cost $\$ 15$ per participant. Table 6 shows the per person program costs for the total number recruited $(n=246)$, the study sample $(n=188)$, and at full capacity for the 34 Living Well replications $(\mathrm{n}=408)$.

Table 6: Per Person Living Well Program Costs

\begin{tabular}{|l|c|c|c|c||}
\hline & $\begin{array}{c}\text { Replications and } \\
\text { Training Costs }\end{array}$ & Materials & $\begin{array}{c}\text { Total Program } \\
\text { Costs }\end{array}$ & Costs per Person \\
\hline $\begin{array}{l}\text { Total Recruited } \\
\mathrm{n}=246\end{array}$ & $\$ 109,148$ & $\$ 3,690$ & $\$ 112,838$ & $\$ 459$ \\
\hline $\begin{array}{l}\text { Study Sample } \\
\mathrm{n}=188\end{array}$ & $\$ 109,148$ & $\$ 2,820$ & $\$ 111,968$ & $\$ 596$ \\
\hline $\begin{array}{l}\text { Full Capacity } \\
\mathrm{n}=408\end{array}$ & $\$ 109,148$ & $\$ 6,120$ & $\$ 115,268$ & $\$ 283$ \\
\hline
\end{tabular}

Program costs and associated net benefits are obviously shaped by recruitment levels. For this particular study, program costs for the188 study participants are used since program outcomes data is missing for the remaining 58 individuals who were recruited into the Living Well intervention. Although this may overstate the per person program costs of implementing the program, at least program costs and outcomes are assessed for the same sample.

Data was not collected for out-of-pocket costs incurred by Living Well participants. These additional costs might include travel expenses (mileage, lodging, meals, public accessible transportation, hired drivers), attendant costs (personal assistants, readers, interpreter services, or childcare), and foregone wages/leisure. Although it is possible to impute a lost production value for Living Well participants, $83.8 \%$ of participants were not employed and only $5.4 \%$ of participants were employed full-time. 
Given the profile of the study population and the limitations of the survey instrument, personal costs to participate in the Living Well program are not included.

\section{Program Outcomes/Benefits}

Program outcomes are measured as changes in medical care utilization costs. In each survey round, participant data were collected for number of hospital days, doctors visits, outpatient visits, and emergency room visits in the prior two months. Additional information was collected about tests and therapies. S. Senninger (personal communication, May 15, 2000) at the Bureau of Business and Economic Research at The University of Montana provided average Medicare cost estimates for most health care utilization measures, using 1998 data from aggregated Medicare reimbursement rates, national outpatient revenue summary data, and physician reimbursement rates from the BESS system (a database from Health Care Financing Administration, Bureau of Data Management and Strategy). Table 7 lists the estimated unit cost of each type of visit or service. All costs were estimated to have increased between 1998 and 1999 by $3.7 \%$, the rate of increase in the medical care component of the Consumer Price Index (2000).

\section{Table 7: Cost Estimates for Medicare}

\begin{tabular}{|l|c|c||}
\hline \multicolumn{1}{|c|}{ Type of Service } & 1998 Cost per Unit & 1999 Cost per Unit \\
\hline \hline Day of inpatient (hospital) care & $\$ 1,073$ & $\$ 1,113$ \\
\hline Emergency room visit & $\$ 157$ & $\$ 163$ \\
\hline Hospital outpatient visit & $\$ 419$ & $\$ 435$ \\
\hline Physician visit & $\$ 89$ & $\$ 92$ \\
\hline
\end{tabular}




\begin{tabular}{|l|c|c|}
\hline \multicolumn{1}{|c|}{ Type of Service } & 1998 Cost per Unit & 1999 Cost per Unit \\
\hline \hline Tests and Therapies (non-routine) & & \\
\hline Blood test & $\$ 83$ & $\$ 86$ \\
\hline Urine test & $\$ 53$ & $\$ 55$ \\
\hline EKG & $\$ 70$ & $\$ 73$ \\
\hline X-Ray & $\$ 120$ & $\$ 124$ \\
\hline Prostate exam & $\$ 53$ & $\$ 55$ \\
\hline Pap smear & $\$ 53$ & $\$ 55$ \\
\hline Mammogram & $\$ 53$ & $\$ 55$ \\
\hline Respiratory therapy & $\$ 56$ & $\$ 58$ \\
\hline Occupational therapy & $\$ 66$ & $\$ 68$ \\
\hline Physical therapy & $\$ 52$ & $\$ 54$ \\
\hline Chiropractor & $\$ 50$ & $\$ 52$ \\
\hline Massage therapy & $\$ 50$ & $\$ 52$ \\
\hline Acupuncture & $\$ 50$ & $\$ 52$ \\
\hline Counseling & $\$ 97$ & $\$ 101$ \\
\hline Skilled nursing services & $\$ 90$ & $\$ 93$ \\
\hline
\end{tabular}

In addition to the unit cost rates reported in Table 7, low- and high-cost insurance reimbursement schedules were constructed. The low schedule, calculated at $70 \%$ of Medicare costs, was meant to serve as a proxy for Medicaid reimbursement rates. Likewise, a high cost schedule, calculated at $130 \%$ of Medicare costs, represents private insurance reimbursement rates. Analyzing results based on these three different reimbursement schedules shows the returns of program outcomes for the three primary types of insurance providers - Medicaid, Medicare, and private insurance. 
The low and high cost reimbursement schedules are based on recommendations from the literature. Norton (1995) found that nationally Medicaid reimbursement rates are $73 \%$ of Medicare rates. Although Medicaid reimbursement figures vary across states, the average Medicaid rates for this study mirror the national average. Table 8 lists the state Medicaid reimbursement rate at each Living Well implementation site, expressed as a percent of the national average.

Table 8: State Medicaid Reimbursement Compared to National Average

\begin{tabular}{|l|c|}
\hline \multicolumn{1}{|c|}{ Location } & \multicolumn{1}{|c|}{$\begin{array}{c}\text { State Medicaid Reimbursement } \\
\text { Divided By National Average }\end{array}$} \\
\hline \hline Missouri (Intervention 1) & $95 \%$ \\
\hline Missouri (Intervention 2) & $95 \%$ \\
\hline California & $89 \%$ \\
\hline Texas & $108 \%$ \\
\hline New Hampshire & $102 \%$ \\
\hline Mississippi & $86 \%$ \\
\hline New York & $93 \%$ \\
\hline Montana & $126 \%$ \\
\hline Kansas & $119 \%$ \\
\hline Average of all sites & $101 \%$ \\
\hline
\end{tabular}

Additional sources indicate that Medicaid and Medicare reimbursement rates are substantially lower than private insurance. "Taking billed or gross hospital charges in 1990 as a benchmark, Medicaid is reported to cover only $54 \%$ of charges, followed by Medicare (60\%) and private insurance (86\%)" (Dor \& Farley, 1996, p.16). Although it is difficult to assess an average reimbursement rates across private insurance providers, 
Medicare cost figures were inflated by $30 \%$ for this analysis.

Shi (1993) used a similar cost estimation process when analyzing the cost-benefits of a Health Risk Appraisal program in California. Shi constructed low, medium, and high direct medical cost rates using data from the National Center for Health Statistics. Medium costs were collected from available state cost averages and his low and high medical cost rates were decreased and increased by $40 \%$ to develop a range. Kruger, et al. (1998) used threshold values to demonstrate the strength of his model. For costbenefit values such as programmatic and health care utilization costs he calculated threshold values at which the program becomes cost incurring when maintaining base case values for other measures.

Table 9 provides cost estimates for the Living Well study for low, medium, and high cost structures for 1998 and 1999.

Table 9: Low, Medium, and High Cost Estimates

\begin{tabular}{|c|c|c|c|c|c|c|}
\hline Type of Service & $\begin{array}{l}1998 \\
\text { Low }\end{array}$ & $\begin{array}{c}1998 \\
\text { Medium } \\
\end{array}$ & $\begin{array}{l}1998 \\
\text { High }\end{array}$ & $\begin{array}{l}1999 \\
\text { Low }\end{array}$ & $\begin{array}{c}1999 \\
\text { Medium }\end{array}$ & $\begin{array}{l}1999 \\
\text { High } \\
\end{array}$ \\
\hline Day of hospital care & $\$ 751$ & $\$ 1,073$ & $\$ 1,395$ & $\$ 779$ & $\$ 1,113$ & $\$ 1,447$ \\
\hline Emergency room visit & $\$ 110$ & $\$ 157$ & $\$ 204$ & $\$ 114$ & $\$ 163$ & $\$ 212$ \\
\hline Hospital outpatient visit & $\$ 293$ & $\$ 419$ & $\$ 545$ & $\$ 304$ & $\$ 435$ & $\$ 565$ \\
\hline Physician visit & $\$ 62$ & $\$ 89$ & $\$ 116$ & $\$ 65$ & $\$ 92$ & $\$ 120$ \\
\hline \multicolumn{7}{|l|}{ Tests and Therapies } \\
\hline Blood test & $\$ 58$ & $\$ 83$ & $\$ 108$ & $\$ 60$ & $\$ 86$ & $\$ 112$ \\
\hline Urine test & $\$ 37$ & $\$ 53$ & $\$ 69$ & $\$ 38$ & $\$ 55$ & $\$ 71$ \\
\hline EKG & $\$ 49$ & $\$ 70$ & $\$ 91$ & $\$ 51$ & $\$ 73$ & $\$ 94$ \\
\hline X-Ray & $\$ 84$ & $\$ 120$ & $\$ 156$ & $\$ 87$ & $\$ 124$ & $\$ 162$ \\
\hline Prostate exam & $\$ 37$ & $\$ 53$ & $\$ 69$ & $\$ 38$ & $\$ 55$ & $\$ 71$ \\
\hline
\end{tabular}




\begin{tabular}{|l|c|c|c|c|c|c||}
\hline \multicolumn{1}{|c|}{ Type of Service } & $\begin{array}{c}1998 \\
\text { Low }\end{array}$ & $\begin{array}{c}1998 \\
\text { Medium }\end{array}$ & $\begin{array}{c}1998 \\
\text { High }\end{array}$ & $\begin{array}{c}1999 \\
\text { Low }\end{array}$ & $\begin{array}{c}1999 \\
\text { Medium }\end{array}$ & $\begin{array}{c}1999 \\
\text { High }\end{array}$ \\
\hline \hline Pap smear & $\$ 37$ & $\$ 53$ & $\$ 69$ & $\$ 38$ & $\$ 55$ & $\$ 71$ \\
\hline Mammogram & $\$ 37$ & $\$ 53$ & $\$ 69$ & $\$ 38$ & $\$ 55$ & $\$ 71$ \\
\hline Respiratory therapy & $\$ 39$ & $\$ 56$ & $\$ 73$ & $\$ 41$ & $\$ 58$ & $\$ 75$ \\
\hline Occupational therapy & $\$ 46$ & $\$ 66$ & $\$ 86$ & $\$ 48$ & $\$ 68$ & $\$ 89$ \\
\hline Physical therapy & $\$ 36$ & $\$ 52$ & $\$ 68$ & $\$ 38$ & $\$ 54$ & $\$ 70$ \\
\hline Chiropractor & $\$ 35$ & $\$ 50$ & $\$ 65$ & $\$ 36$ & $\$ 52$ & $\$ 67$ \\
\hline Massage therapy & $\$ 35$ & $\$ 50$ & $\$ 65$ & $\$ 36$ & $\$ 52$ & $\$ 67$ \\
\hline Acupuncture & $\$ 35$ & $\$ 50$ & $\$ 65$ & $\$ 36$ & $\$ 52$ & $\$ 67$ \\
\hline Counseling & $\$ 68$ & $\$ 97$ & $\$ 126$ & $\$ 70$ & $\$ 101$ & $\$ 131$ \\
\hline Skilled nursing services & $\$ 63$ & $\$ 90$ & $\$ 117$ & $\$ 65$ & $\$ 93$ & $\$ 121$ \\
\hline
\end{tabular}

Additional program outcomes/benefits might include changes in travel expenses (mileage, lodging, meals, public accessible transportation, hired drivers), attendant costs (personal assistants, readers, interpreter services, or childcare), and foregone wages/leisure for time spent receiving medical services. Just as participant costs are excluded from Living Well implementation costs, participant outcomes, other than changes in utilization, are excluded from program benefits. 


\section{Chapter 4: Cost Benefit Analysis - Results}

This chapter, which presents the results of the cost-benefit analysis of the Living Well program, is divided into three sections. The first section examines the stability of healthcare utilization between the extended baseline and comparison group. This analysis helps inform the degree to which changes in health care utilization are impacted by the Living Well program versus other external factors.

The next section reports net benefits over several time periods including immediate post-intervention, 2 months post-intervention, and 4 months post-intervention. One year-post intervention data is also analyzed to show any rebound effects or trends back to baseline health care utilization. With this data, cost-incurring versus costsavings outcome segments of the Living Well program are demonstrated.

Finally, non-quantifiable aspects of the program are analyzed to show how the Living Well program impacts quality of life. Quality of life measures include the sum of secondary conditions, health promoting lifestyle, life satisfaction, and overall quality of life variables. These three result sections combine to make a strong recommendation to third party payers about the feasibility of providing cost reimbursement for health promotion activities.

\section{Extended Baseline Comparisons}

The Living Well study did not have a true control group. Instead, approximately half of the participants were randomly assigned to wait two months before receiving the Living Well workshop and provided an extra data collection point two months prior to intervention. Without a true control measure, there is less confidence about the impacts 
attributable to the Living Well intervention versus other external factors. However, an extended baseline does provide information about the stability of health care utilization prior to the intervention. Health care utilization stability provides information about the confidence that significant outcome differences are attributable to the Living Well ịntervention.

\section{Extended Baseline Comparisons}

In the absence of a control measure, baseline medical costs and conditions are evaluated to detect trends in health care utilization. The extended baseline cohort of seventy-nine participants provided data at 2-months pre-intervention (survey A) and immediate pre-intervention (survey B). Data from these two points are compared to determine if overall health, lifestyle, and medical care utilization changed significantly during the two months prior to intervention.

Overall health is summarized by the sum of secondary conditions. The sum of secondary conditions score is simply the arithmetic sum of an individual's ratings on each secondary condition. This score can range from 0 (no limitation on any secondary condition) to 129 (a 3 rating on each of the 43 secondary conditions included). Although this score does not adequately describe all health aspects, it does serve as a proxy for comorbidities in the study sample.

Lifestyle attributes or behaviors are summarized using indices of the Health Promoting Lifestyle profile. Lifestyle attributes impact how people manage their health. The Health Promoting Lifestyle Profile is broken into sub-scales of lifestyle characteristics including health responsibility, physical activity, nutrition, self- 
actualization, interpersonal support, and stress management. In addition to sub-scales for each lifestyle characteristic, scores can be averaged across attributes to generate a total lifestyle score. For the purposes of comparing the paired samples, the total lifestyle score is shown.

Health care costs are measured using health care utilization rates multiplied by unit costs. Health care costs include clinical services (doctors visits, outpatient visits, emergency room visits, and hospital days) and two aggregated cost calculations. The health care utilization comparisons use 1998 and 1999 Medicare cost estimates. The first aggregate variable includes costs associated with hospital visits, emergency room visits, outpatient visits, and doctors visits. The second variable has additional test and therapy costs. The aggregate variables are defined as:

COST1 $=\quad$ hospital costs + er costs + outpatient costs + physician costs $\operatorname{COST} 2=\operatorname{COST} 1+$ test costs (includes blood tests, urine tests, EKGs, X-Rays, prostate exams, pap smears, mammograms.) + therapy costs (includes respiratory therapy, occupational therapy, physical therapy, chiropractor, massage therapy, acupuncture, counseling, and skilled nursing services.)

If any element of an aggregate variable is missing, the value of the aggregate is treated as missing as well.

Cost calculations are presented for both cohort and trimmed data. Trimmed data excludes outliers greater than three standard deviations from the mean. A trimmed data set is included because analysis of the entire data set introduced extremely large variance into the sample and there was evidence of double counting between high rates of 
hospitalization, tests, and therapies.

Using paired samples t-tests for equality of means (for the sum of secondary conditions and health promoting lifestyle scale), and Wilcoxon signed ranks tests (for health care utilization cost data), Table 10 shows comparisons between the extended baseline group at A (2-months pre-intervention) and B (immediate pre-intervention) time periods. The Wilcoxon non-parametric test is used for medical utilization cost data because the assumption of normality is violated.

Table 10: Extended Baseline Comparison (Survey $A$ and Survey B)

\begin{tabular}{|l|c|c|c|c|c|c||}
\hline \multicolumn{1}{|c|}{ Health Characteristics } & $\begin{array}{c}\text { Mean A } \\
\text { (pre-pre) }\end{array}$ & $\begin{array}{c}\text { Mean B } \\
\text { (pre) }\end{array}$ & $\begin{array}{c}\text { Sig } \\
\text { (2-tailed) }\end{array}$ & $\begin{array}{c}\text { Mean A } \\
\text { Trimmed }\end{array}$ & $\begin{array}{c}\text { Mean B } \\
\text { Trimmed }\end{array}$ & $\begin{array}{c}\text { Sig. } \\
\text { (2-tailed) }\end{array}$ \\
\hline $\begin{array}{l}\text { Sum of secondary } \\
\text { conditions (n=79) }\end{array}$ & 31.73 & 29.85 & .058 & NA & NA & NA \\
\hline $\begin{array}{l}\text { Health Promoting Lifestyle } \\
\text { Scale (n=73) }\end{array}$ & 2.52 & 2.47 & .153 & NA & NA & NA \\
\hline $\begin{array}{l}\text { Hospital Day Visits } \\
(\mathrm{n}=76 / 74)\end{array}$ & $\$ 579$ & $\$ 935$ & .480 & $\$ 189$ & $\$ 337$ & .773 \\
\hline ER Visits ( $=78 / 76)$ & $\$ 43$ & $\$ 41$ & .912 & $\$ 29$ & $\$ 36$ & .417 \\
\hline Outpatient Visits ( $=77 / 73)$ & $\$ 160$ & $\$ 167$ & .715 & $\$ 77$ & $\$ 100$ & .548 \\
\hline Doctor visits (n=75/72) & $\$ 182$ & $\$ 156$ & .081 & $\$ 152$ & $\$ 138$ & .207 \\
\hline COST1 $(\mathrm{n}=73 / 65)$ & $\$ 986$ & $\$ 1,334$ & .824 & $\$ 457$ & $\$ 582$ & .826 \\
\hline COST2 (n=49/25) & $\$ 2,253$ & $\$ 2,252$ & .695 & $\$ 775$ & $\$ 548$ & .808 \\
\hline
\end{tabular}

Although differences in the sum of secondary conditions approach significance in the paired sample, the Health Promoting Lifestyle scale and medical care utilization costs are similar across the extended baseline. Since the results show no significant change in costs for the extended baseline, significant reductions in medical care costs after 
participation in the program can be more confidently attributed to the Living Well intervention.

The differences between sum of secondary conditions at A ( 2 months preintervention) and B (immediate pre-intervention) can be explained in part by the Hawthorne effect. As described in the literature, the Hawthorne effect occurs when individuals overstate conditions based on how they expect the intervention to work (Shi, 1993; Bertera, 1993). Extended baseline participants may have overstated their health conditions at time A because they felt some urgency about getting into the program. It makes sense that the Hawthorne effect would occur for sum of secondary conditions rather than medical care utilization rates since perceived limitation is more subjective than concrete questions about healthcare utilization.

Doctors visits for untrimmed data also approached significance using the Wilcoxen (nonparametric) signed ranks test. This result was not replicated when data was trimmed to 3 standard deviations from the mean. The data trim resulted in the exclusion of 3 out of 75 cases or $4 \%$ of the data. Although there were differences between doctors visits across the extended baseline, this trend was not replicated for other health care utilization cost variables.

The majority of cases for COST2 had to be thrown out due to missing data in the test and therapy sections. Although the data show no trend toward group differences, there is a risk of making a Type 2 error since much of the data is excluded from analysis.

\section{Extended Baseline and Comparison Groups}

Prior to collapsing the extended baseline group with the larger comparison 
sample, the two sub-samples are compared to determine if they are similar or have factors that differ in ways for which statistical control is necessary. Table 11 compares the demographics between the extended baseline group ( $\mathrm{AB}$ group) and participants who immediately entered the Living Well intervention after the recruitment phase (B group). Tests for significant differences between groups include independent group t-tests for continuous variables and Pearson Chi-Square tests for dichotomous variables. The two groups did not show significant differences in any of the demographic variables including age at intervention, years with a disability, location, gender, disability type, years of education, marital status, race, employment status, and health care insurance coverage.

Table 11: Demographic Comparisons Between Extended Baseline and Comparison

\begin{tabular}{|c|c|c|c|c|c|}
\hline Demographics & $\mathrm{AB}$ Mean & B Mean & $\begin{array}{c}\text { AB Valid } \\
\text { Percent }\end{array}$ & $\begin{array}{l}\text { B Valid } \\
\text { Percent }\end{array}$ & $\begin{array}{c}\text { Sig. } \\
\text { (2-tailed) }\end{array}$ \\
\hline Age at intervention $(n=78 / 109)$ & 44.53 & 46.00 & & & .460 \\
\hline Years with disability $(n=63 / 88)$ & 17.43 & 17.52 & & & .971 \\
\hline $\begin{array}{c}\text { Location }(\mathrm{n}=\mathbf{7 8 / 1 0 9 )} \\
\text { Rural } \\
\text { Urban }\end{array}$ & & & $\begin{array}{l}26.9 \\
73.1\end{array}$ & $\begin{array}{l}33.9 \\
66.1\end{array}$ & .306 \\
\hline $\begin{array}{c}\text { Gender }(\mathrm{n}=\mathbf{7 8 / 1 0 9 )} \\
\text { Male } \\
\text { Female }\end{array}$ & & & $\begin{array}{l}38.5 \\
61.5\end{array}$ & $\begin{array}{l}33.9 \\
66.1\end{array}$ & .525 \\
\hline $\begin{array}{c}\text { Disability Type }(n=76 / 107) \\
\text { Stable } \\
\text { Degenerative }\end{array}$ & & & $\begin{array}{l}46.1 \\
53.9\end{array}$ & $\begin{array}{l}49.5 \\
50.5\end{array}$ & .642 \\
\hline Years of Education ( $n=75 / 105$ ) & 13.76 & 13.66 & & & .840 \\
\hline $\begin{array}{c}\text { Marital Status }(\mathrm{n}=79 / 107) \\
\text { Not married } \\
\text { Married }\end{array}$ & & & $\begin{array}{l}62.0 \\
38.0\end{array}$ & $\begin{array}{l}64.5 \\
35.5\end{array}$ & .731 \\
\hline
\end{tabular}




\begin{tabular}{|c|c|c|c|c|c|}
\hline Demographics & AB Mean & B Mean & $\begin{array}{c}\text { AB Valid } \\
\text { Percent }\end{array}$ & $\begin{array}{l}\text { B Valid } \\
\text { Percent }\end{array}$ & $\begin{array}{c}\text { Sig. } \\
\text { (2-tailed) }\end{array}$ \\
\hline $\begin{array}{l}\text { Race }(\boldsymbol{n}=79 / 109) \\
\text { White } \\
\text { Black/African American } \\
\text { Hawaiian/Pacific } \\
\text { Islander } \\
\text { Asian } \\
\text { American Indian/Alaska } \\
\text { Native }\end{array}$ & & & $\begin{array}{c}83.5 \\
11.4 \\
1.3 \\
0 \\
2.5\end{array}$ & $\begin{array}{c}81.7 \\
15.6 \\
0 \\
.9 \\
2.8\end{array}$ & $\begin{array}{l}.736 \\
.410 \\
.239 \\
.393 \\
.926\end{array}$ \\
\hline $\begin{array}{l}\text { Ethnicity }(n=79 / 109) \\
\text { Hispanic or Latino } \\
\text { Other }\end{array}$ & & & $\begin{array}{l}2.5 \\
97.5\end{array}$ & $\begin{array}{c}3.7 \\
96.3\end{array}$ & .661 \\
\hline $\begin{array}{l}\text { Employment Status ( } \mathrm{n}=78 / 107 \text { ) } \\
\text { Employed (part or full } \\
\text { time) } \\
\text { Not employed }\end{array}$ & & & $\begin{array}{l}15.4 \\
84.6\end{array}$ & $\begin{array}{l}16.8 \\
83.2\end{array}$ & .793 \\
\hline $\begin{array}{l}\text { Insurance Status }(\mathbf{n}=78 / 106) \\
\text { Medicaid } \\
\text { Medicare } \\
\text { Private } \\
\text { Veterans Administration } \\
\text { No Insurance }\end{array}$ & & & $\begin{array}{c}61.5 \\
47.4 \\
30.8 \\
5.1 \\
2.6\end{array}$ & $\begin{array}{c}51.9 \\
56.6 \\
39.6 \\
4.7 \\
6.6\end{array}$ & $\begin{array}{l}.192 \\
.218 \\
.209 \\
.216 \\
.209\end{array}$ \\
\hline
\end{tabular}

Additionally, the two groups were similar in terms of overall health, measured by the sum of secondary conditions, health promoting lifestyle, and health care utilization costs. Using independent samples t-tests (for sum of secondary conditions and health promoting lifestyle) and Mann-Whitney nonparametric tests (for health care utilization cost data), extended baseline (AB) and comparison (B) groups were not statistically different at pre-intervention (Survey B). 
Table 12: Health Characteristic Comparisons Between Extended Baseline and

Comparison

\begin{tabular}{|l|c|c|c|c|c|c||}
\hline \multicolumn{1}{|c|}{ Health Characteristics } & ABMean & B Mean & $\begin{array}{c}\text { Sig } \\
\text { (2-tailed) }\end{array}$ & $\begin{array}{c}\text { AB Mean } \\
\text { Trimmed }\end{array}$ & $\begin{array}{c}\text { B Mean } \\
\text { Trimmed }\end{array}$ & $\begin{array}{c}\text { Sig. } \\
\text { (2-tailed) }\end{array}$ \\
\hline $\begin{array}{l}\text { Sum of secondary } \\
\text { conditions (n=79/109) }\end{array}$ & 29.85 & 29.83 & .993 & NA & NA & NA \\
\hline $\begin{array}{l}\text { Health Promoting Lifestyle } \\
\text { Scale (n=74/101) }\end{array}$ & 2.47 & 2.50 & .657 & NA & NA & NA \\
\hline $\begin{array}{l}\text { Hospital Day Visits } \\
(n=76 / 109-75 / 105)\end{array}$ & $\$ 935$ & $\$ 2,239$ & .455 & $\$ 332$ & $\$ 607$ & .755 \\
\hline $\begin{array}{l}\text { ER Visits } \\
(n=78 / 108-78 / 106)\end{array}$ & $\$ 41$ & $\$ 57$ & .761 & $\$ 41$ & $\$ 33$ & .985 \\
\hline $\begin{array}{l}\text { Outpatient Visits } \\
(n=77 / 100-75 / 105)\end{array}$ & $\$ 167$ & $\$ 199$ & .271 & $\$ 102$ & $\$ 171$ & .195 \\
\hline $\begin{array}{l}\text { Doctor visits } \\
(n=77 / 100-77 / 96)\end{array}$ & $\$ 159$ & $\$ 218$ & .329 & $\$ 159$ & $\$ 178$ & .598 \\
\hline $\begin{array}{l}\text { COST1 } \\
(n=75 / 98-72 / 86)\end{array}$ & $\$ 1,306$ & $\$ 2,881$ & .356 & $\$ 594$ & $\$ 965$ & .911 \\
\hline $\begin{array}{l}\text { COST2 } \\
(\mathrm{n}=61 / 79-43 / 45)\end{array}$ & $\$ 1,940$ & $\$ 4,962$ & $.005 * *$ & $\$ 639$ & $\$ 1,297$ & .215 \\
\hline
\end{tabular}

${ }^{* *}$ significant at the .01 level

Non-trimmed data did show significant differences between the $A B$ and $B$ groups

for the COST2 variable but this result was not replicated when outliers were excluded.

Once again, results from $\operatorname{COST} 2$ are suspect since a large portion of the data is excluded due to missing data in tests and services. Although the extended baseline group typically had lower medical costs at pre-intervention, these differences were much smaller when controlling for extreme values (trimmed data set) and were not statistically different.

Since the extended baseline and comparison groups are indistinguishable and health care utilization costs were stable across the extended baseline period, it follows 
that any detectable trends during the intervention phase of this study are attributable to participation in the Living Well workshop. For the remainder of this study, the extended baseline and comparison groups are collapsed to form a study cohort of 188 participants.

\section{The Cost Benefit Analysis}

The net benefits associated with the Living Well workshop are analyzed using the cohort of 188 individuals who completed both immediate pre-intervention (B) and immediate post-intervention (C) questionnaires. Although there was attrition across subsequent data collection points, the $\mathrm{B}$ to $\mathrm{C}$ criteria maintains a sample which, at the very least, participated in the Living Well intervention.

The net benefit equation equals program outcomes, measured as change in medical care utilization costs, minus program costs. Changes in medical care costs are calculated in two month segments to show short and long term health care utilization changes. The following net benefit equation shows return on program costs during the first six months after Living Well implementation.

$$
\begin{aligned}
\text { NB }= & \text { Program Benefits (outcomes) }- \text { Program Costs }(\mathrm{PC}) \\
= & {[\text { Health Costs }(6 \text { months prior })-\text { Heath costs }(6 \text { months post })]-\mathrm{PC} } \\
=\quad & {[(\text { Health Costs (immediate pre }) * 3)-\text { Health Costs (immediate } } \\
& \text { post })- \text { Health Costs }(2 \text { months post })-\text { Health Costs }(4 \text { months } \\
& \text { post })]-\mathrm{PC}
\end{aligned}
$$

Results are presented for both trimmed and un-trimmed data and further analysis examines a high medical use subgroup, defined as individuals who incurred at least some costs in the two months prior to the Living Well intervention. 
High medical use data is included for two reasons. First, high risk individuals potentially benefit more from health promotion since they may have medical conditions that are more responsive to intervention strategies (Pelletier, 1996; Kaman, \& Patton, 1992). Second, the net benefit equation overstates health utilization at pre-intervention since the immediate pre-intervention (B) cost measure is multiplied by three to serve as a proxy for medical care across a six-month pre-intervention period. By excluding individuals who have no health care at point $B$, the risk of understating health utilization prior to the intervention is minimized. The high medical care subgroup is constructed using trimmed data to minimize the impacts of exceptionally high medical costs as well.

\section{Net Benefits for Low, Medium, and High Medical Care Costs}

For each group (entire cohort, trimmed and high medical users), net benefit equations are constructed for three medical cost structures: low medical costs (calculated at $70 \%$ of Medicare cost projections), medium medical costs (Medicare cost projections), and high medical costs (calculated at $130 \%$ of Medicare cost projections). This range of costs is meant to demonstrate the strength of the Living Well workshop for different insurance types - Medicaid (low cost), Medicare (medium cost), and private insurance (high cost). Table 13 shows the mean medical cost expenditures at each data collection point included in the net benefit equation. These mean values are used to construct the program outcome segment of the net benefit equations. 
Table 13: Mean Health Care Expenditures

\begin{tabular}{|c|c|c|c|c|c|c|c|c|c|}
\hline \multirow[t]{2}{*}{ Health Care Utilization } & \multicolumn{3}{|c|}{$\begin{array}{l}\text { Mean Expenditures } \\
\text { Entire Cohort }\end{array}$} & \multicolumn{3}{|c|}{$\begin{array}{l}\text { Mean Expenditures } \\
\text { Trimmed Data }\end{array}$} & \multicolumn{3}{|c|}{$\begin{array}{l}\text { Mean Expenditures } \\
\text { High Users (Trimmed) }\end{array}$} \\
\hline & Low & Med. & High & Low & Med. & High & Low & Med. & High \\
\hline COST1 at $B$ & $\$ 1,539$ & $\$ 2,198$ & $\$ 2,814$ & $\$ 557$ & $\$ 796$ & $\$ 1,017$ & $\$ 815$ & $\$ 1,164$ & $\$ 1.487$ \\
\hline CosT1 at $\mathrm{C}$ & $\$ 501$ & $\$ 716$ & $\$ 920$ & $\$ 238$ & $\$ 340$ & $\$ 436$ & $\$ 248$ & $\$ 355$ & $\$ 455$ \\
\hline $\cos 11$ at $D$ & $\$ 871$ & $\$ 1,245$ & $\$ 1,604$ & $\$ 318$ & $\$ 455$ & $\$ 585$ & $\$ 342$ & $\$ 489$ & $\$ 629$ \\
\hline $\operatorname{cosT} 1$ at $\mathrm{E}$ & $\$ 915$ & $\$ 1,308$ & $\$ 1,687$ & $\$ 286$ & $\$ 408$ & $\$ 526$ & $\$ 397$ & $\$ 568$ & $\$ 732$ \\
\hline
\end{tabular}

Program costs, as described in the Methods and Analysis chapter, totaled

$\$ 111,968$ or $\$ 596$ per person to implement the Living Well workshop for the study cohort.

This intervention cost is subtracted from program outcomes for low, medium, and high cost values in Tables 14, 15, and 16 .

Table 14: Net Benefits - Entire Cohort of 188 Participants

\begin{tabular}{||l|c|c|c|}
\hline \multicolumn{1}{|c|}{ Cost Structure } & Program Outcomes & Program Costs & Net Benefit \\
\hline \hline Low Cost & $\$ 2,330$ per person & $\$ 596$ per person & $\$ 1,734$ per person \\
\hline Medium Cost & $\$ 3,325$ per person & $\$ 596$ per person & $\$ 2,729$ per person \\
\hline High Cost & $\$ 4,231$ per person & $\$ 596$ per person & $\$ 3,635$ per person \\
\hline
\end{tabular}

Table 15: Net Benefits - Trimmed Data Set

\begin{tabular}{|l|c|c|c|}
\hline \multicolumn{1}{|c|}{ Cost Structure } & Program Outcomes & Program Costs & Net Benefit \\
\hline \hline Low Cost & $\$ 829$ per person & $\$ 596$ per person & $\$ 233$ per person \\
\hline Medium Cost & $\$ 1,185$ per person & $\$ 596$ per person & $\$ 589$ per person \\
\hline High Cost & $\$ 1,504$ per person & $\$ 596$ per person & $\$ 908$ per person \\
\hline
\end{tabular}


Table 16: Net Benefits - High Use Participants

\begin{tabular}{||l|c|c|c||}
\hline \multicolumn{1}{|c|}{ Cost Structure } & Program Outcomes & Program Costs & Net Benefit \\
\hline \hline Low Cost & \$1,458 per person & \$596 per person & \$862 per person \\
\hline Medium Cost & $\$ 2,080$ per person & \$596 per person & $\$ 1,484$ per person \\
\hline High Cost & $\$ 2,645$ per person & \$596 per person & $\$ 2,049$ per person \\
\hline
\end{tabular}

Results from the Net Benefit equations show a remarkable payback for the Living Well intervention. For all sub-samples (cohort, trimmed, and high medical use participants), the Living Well intervention resulted in positive net benefits in the first six months following program implementation. Program costs are completely realized in the first two month interval when using data from the entire cohort. More conservative estimates, using the trimmed data set, show positive net benefits in the first two months for high cost values, first four months for medium cost values, and first six months for low cost rates. Finally, the high medical care users have positive net benefits in the first two months for medium and high cost estimates, and positive net benefits in the first four months for low cost estimates. This data provides concrete evidence for insurance companies to use when considering reimbursement of health promotion programs. Additionally, the net benefits would be even greater if program implementation costs were averaged across the total number of participants recruited into the program $(n=$ 246).

Before accepting the net benefit results at face value, however, it is important to establish that changes in medical care utilization rates are statistically significant between 
the baseline (survey B) and subsequent data points (surveys C, D, and E). The nonparametric Wilcoxon signed ranks test compares health costs across time for the cohort, trimmed, and high medical care groups. This analysis is conducted using only medium cost estimates since statistical significance for low and high unit costs replicates medium cost results.

Table 17: Paired Sample Tests - Pre-Intervention to Post-Intervention

\begin{tabular}{|c|c|c|c|}
\hline Health Care Utilization & $\begin{array}{c}\text { Cohort Data } \\
\text { Two-Tailed Sig. }\end{array}$ & $\begin{array}{l}\text { Trimmed Data } \\
\text { Two-Tailed Sig. }\end{array}$ & $\begin{array}{c}\text { High Users (Trimmed) } \\
\text { Two-Tailed Sig. }\end{array}$ \\
\hline $\begin{array}{l}\text { Hospital Costs } \\
\mathrm{B} \text { to } \mathrm{C}\end{array}$ & $\begin{array}{c}.012^{*} \\
\mathrm{n}=184\end{array}$ & $\begin{array}{c}.028^{*} \\
\mathrm{n}=179\end{array}$ & $\begin{array}{c}.017^{*} \\
\mathrm{n}=107\end{array}$ \\
\hline $\begin{array}{l}\text { Emergency Room Costs } \\
\text { B to C }\end{array}$ & $\begin{array}{c}.257 \\
\mathrm{n}=185\end{array}$ & $\begin{array}{c}.020 * \\
\mathrm{n}=174\end{array}$ & $\begin{array}{c}.025^{*} \\
\mathrm{n}=103\end{array}$ \\
\hline $\begin{array}{l}\text { Outpatient Costs } \\
\text { B to C }\end{array}$ & $\begin{array}{c}.010^{*} \\
\mathrm{n}=181\end{array}$ & $\begin{array}{l}.003 * * \\
\mathrm{n}=173\end{array}$ & $\begin{array}{l}.006 * * \\
\mathrm{n}=103\end{array}$ \\
\hline $\begin{array}{l}\text { Doctor Visit Costs } \\
\mathrm{B} \text { to C }\end{array}$ & $\begin{array}{c}.263 \\
\mathrm{n}=160\end{array}$ & $\begin{array}{c}.204 \\
\mathrm{n}=154\end{array}$ & $\begin{array}{l}.000 * * \\
\mathrm{n}=98\end{array}$ \\
\hline $\begin{array}{l}\text { CosT1 } \\
\text { B to C }\end{array}$ & $\begin{array}{l}.009 * * \\
\mathrm{n}=154\end{array}$ & $\begin{array}{l}.003 * * \\
n=133\end{array}$ & $\begin{array}{l}.000^{* *} \\
\mathrm{n}=90\end{array}$ \\
\hline $\begin{array}{l}\operatorname{COST2} \\
\mathrm{B} \text { to } \mathrm{C}\end{array}$ & $\begin{array}{c}.141 \\
\mathrm{n}=103\end{array}$ & $\begin{array}{l}.124 \\
\mathrm{n}=47\end{array}$ & $\begin{array}{l}.039^{*} \\
\mathrm{n}=35\end{array}$ \\
\hline $\begin{array}{l}\text { Hospital Costs } \\
\text { B to D }\end{array}$ & $\begin{array}{c}.318 \\
n=162\end{array}$ & $\begin{array}{c}.380 \\
\mathrm{n}=157\end{array}$ & $\begin{array}{c}.065 \\
\mathrm{n}=90\end{array}$ \\
\hline $\begin{array}{l}\text { Emergency Costs } \\
B \text { to D }\end{array}$ & $\begin{array}{c}.507 \\
\mathrm{n}=162\end{array}$ & $\begin{array}{c}.219 \\
\mathrm{n}=156\end{array}$ & $\begin{array}{l}.048^{*} \\
\mathrm{n}=89\end{array}$ \\
\hline $\begin{array}{l}\text { Outpatient Costs } \\
\text { B to D }\end{array}$ & $\begin{array}{c}.283 \\
\mathrm{n}=158\end{array}$ & $\begin{array}{c}.193 \\
\mathrm{n}=151\end{array}$ & $\begin{array}{c}.077 \\
\mathrm{n}=86\end{array}$ \\
\hline $\begin{array}{l}\text { Doctor Visit Costs } \\
\text { B to D }\end{array}$ & $\begin{array}{c}.468 \\
\mathrm{n}=137\end{array}$ & $\begin{array}{c}.654 \\
n=131\end{array}$ & $\begin{array}{c}.090 \\
\mathrm{n}=80\end{array}$ \\
\hline $\begin{array}{l}\text { COST1 } \\
B \text { to } D\end{array}$ & $\begin{array}{c}.131 \\
\mathrm{n}=131\end{array}$ & $\begin{array}{c}.279 \\
\mathrm{n}=113\end{array}$ & $\begin{array}{l}.007 * * \\
\mathrm{n}=76\end{array}$ \\
\hline $\begin{array}{l}\text { COST2 } \\
\text { B to } D\end{array}$ & $\begin{array}{c}.095 \\
\mathrm{n}=86\end{array}$ & $\begin{array}{c}.874 \\
\mathrm{n}=36\end{array}$ & $\begin{array}{c}.264 \\
\mathrm{n}=27\end{array}$ \\
\hline
\end{tabular}




\begin{tabular}{|c|c|c|c|}
\hline Health Care Utilization & $\begin{array}{l}\text { Cohort Data } \\
\text { Two-Tailed Sig. }\end{array}$ & $\begin{array}{l}\text { Trimmed Data } \\
\text { Two-Tailed Sig. }\end{array}$ & $\begin{array}{l}\text { High Users (Trimmed) } \\
\text { Two-Tailed Sig. }\end{array}$ \\
\hline $\begin{array}{l}\text { Hospital Costs } \\
B \text { to } E\end{array}$ & $\begin{array}{c}.493 \\
n=148\end{array}$ & $\begin{array}{c}.183 \\
n=141\end{array}$ & $\begin{array}{c}.375 \\
\mathrm{n}=82\end{array}$ \\
\hline $\begin{array}{l}\text { Emergency Costs } \\
B \text { to } E\end{array}$ & $\begin{array}{c}.32 \\
\mathrm{n}=148\end{array}$ & $\begin{array}{c}.089 \\
n=142\end{array}$ & $\begin{array}{l}.325 \\
\mathrm{n}=82\end{array}$ \\
\hline $\begin{array}{l}\text { Outpatient Costs } \\
\mathrm{B} \text { to } \mathrm{E}\end{array}$ & $\begin{array}{c}.070 \\
\mathrm{n}=145\end{array}$ & $\begin{array}{c}.182 \\
n=140\end{array}$ & $\begin{array}{l}.180 \\
\mathrm{n}=84\end{array}$ \\
\hline $\begin{array}{l}\text { Doctor Visit Costs } \\
B \text { to } E\end{array}$ & $\begin{array}{c}.648 \\
\mathrm{n}=128\end{array}$ & $\begin{array}{c}.634 \\
\mathrm{n}=123\end{array}$ & $\begin{array}{l}.152 \\
\mathrm{n}=76\end{array}$ \\
\hline $\begin{array}{l}\text { COST1 } \\
\mathrm{B} \text { to } \mathrm{E}\end{array}$ & $\begin{array}{c}.384 \\
\mathrm{n}=122\end{array}$ & $\begin{array}{c}.506 \\
\mathrm{n}=104\end{array}$ & $\begin{array}{l}.084 \\
\mathrm{n}=70\end{array}$ \\
\hline $\begin{array}{l}\text { COST2 } \\
B \text { to } E\end{array}$ & $\begin{array}{c}.34 \\
\mathrm{n}=77\end{array}$ & $\begin{array}{c}.217 \\
\mathrm{n}=34\end{array}$ & $\begin{array}{c}.313 \\
n=27\end{array}$ \\
\hline
\end{tabular}

* significant at the .05 level

** significant at the .01 level

\section{Immediate Pre (B) to Immediate Post (C)}

The data show significant differences between the immediate-pre (survey B) and immediate-post (survey C) values of aggregate cost variable (COST1) for each of the study samples (cohort, timmed, and high cost groups). Cohort data show significant hospital and outpatient cost decreases; trimmed data show significant hospital, emergency room, and outpatient cost decreases; and high-medical use data show significant decreases for all costs (hospital, emergency room, outpatient, and doctor costs).

Cohort and trimmed data both show non-significant differences in doctors visits. This result can be explained by the maintenance component of health promotion. Increases in doctor visits for wellness activities such as yearly physicals, pap smears, prostate exams, and mammograms may offset other reductions in doctors visits for health related problems. Although the test and therapy aggregate cost variable (COST2) is 
shown, it was not significant for the cohort, and trimmed subsample. COST2 is excluded from future discussion due to large amounts of missing data.

\section{Immediate Pre (B) to Two-Months Post (D) and Four-Months Post (E)}

Significant cost differences are not evident when comparing pre-intervention (survey B) and two-month post intervention (survey D) data for the cohort and trimmed subsample. This trend continues for the pre-intervention and four months postintervention comparison. Although these results are discouraging at first glance, when examining high cost medical users, COST1 is significant at two-months postintervention, and approaches significance (.084) at four-months post-intervention.

\section{One Year Post Intervention Data}

In contrast to significant positive health outcomes during the first two months of the Living Well intervention, and to a lesser degree, during the 2 months and 4 months post intervention, positive cost outcomes from baseline to one-year post intervention are less consistent across the cohort, trimmed, and high medical use groups. Table 18 presents the Wilcoxon signed ranks test for baseline costs compared to one-year postintervention data. Results are presented for the entire cohort, trimmed data, and high medical use sub-samples. 
Table 18: Immediate Pre and One Year Post Health Care Utilization Comparisons

\begin{tabular}{||l|c|c|c||}
\hline \multicolumn{1}{|c|}{ Health Care Utilization } & Cohort Data & Trimmed Data & High Use (Trimmed) \\
\hline $\begin{array}{l}\text { COST1 at B } \\
\text { (Immediate Pre-Intervention) }\end{array}$ & $\begin{array}{c}\mu=\$ 2,198 \\
\mathrm{n}=173\end{array}$ & $\begin{array}{c}\mu=\$ 796 \\
\mathrm{n}=158\end{array}$ & $\begin{array}{c}\mu=\$ 1164 \\
\mathrm{n}=108\end{array}$ \\
\hline $\begin{array}{l}\text { COST1 at F } \\
\text { (One Year Post-Intervention) }\end{array}$ & $\begin{array}{c}\mu=\$ 1318 \\
\mathrm{n}=98\end{array}$ & $\begin{array}{c}\mu=\$ 798 \\
\mathrm{n}=97\end{array}$ & $\begin{array}{c}\mu=\$ 689 \\
\mathrm{n}=58\end{array}$ \\
\hline $\begin{array}{l}\text { COST1 - Paired Sample B to F } \\
\text { 2-Tailed Significance Level }\end{array}$ & $\begin{array}{c}.893 \\
\mathrm{n}=89\end{array}$ & $\begin{array}{c}.966 \\
\mathrm{n}=76\end{array}$ & $\begin{array}{c}.070 \\
\mathrm{n}=50\end{array}$ \\
\hline
\end{tabular}

Trimmed data show a complete return to baseline medical care utilization costs and while cohort data reveals large differences in mean values, they are statistically insignificant. Data from the high medical use sample tells a slightly different story. If individuals had medical costs at baseline, statistically significant differences (significance at .10) are maintained one-year post intervention.

To explore these differences between the cohort and high medical use groups, health and lifestyle attributes are compared using independent samples t-tests. T-tests show statistically different sample values for the sum of secondary conditions $(t=2.171$, $p=.031)$ and health promoting lifestyle scores $(t=2.451, p=.015)$. As one might expect, high medical utilizers report more limitations from secondary conditions. What is more surprising is that they also score higher on the total health promoting lifestyle scale.

Perhaps more frequent and ongoing health problems encourage behavior to maintain or improve health. High medical-users may benefit more from Living Well health promotion strategies because they actively seek ways to improve health and recognize the benefits of long-term behavior changes. 
Comparisons between the baseline and one-year post-intervention cost data support the short payback period represented in the net benefit equation. Although cost comparisons approached significance for the high medical use sub-sample, the cohort and trimmed data show uncertain cost outcomes one-year post intervention.

\section{Confidence Intervals}

Closer examination of the mean, standard deviation, and variance of the cost variables helps explain why significance is rare despite seemingly large differences between utilization costs. In each of the samples, mean values decrease substantially from baseline to immediate post, two-months post, and four-months post cost data. These differences, however, are small when standard deviations average 2 to 4 times the mean. Table 19 shows the mean, standard deviation, and variance of COST1 for the cohort, trimmed, and high-use data sets.

Table 19: COST1 - Mean, Standard Deviation, and Variance

\begin{tabular}{|c|c|c|c|}
\hline Health Care Utilization & Cohort Data & Trimmed Data & High Users (Trimmed) \\
\hline $\begin{array}{l}\text { COSTl at } \mathrm{B} \\
\text { Immediate Pre-Intervention }\end{array}$ & $\begin{array}{c}\mu=\$ 2,198 \\
s d=\$ 8,245 \\
v=67,977,444\end{array}$ & $\begin{array}{c}\mu=\$ 796 \\
\mathbf{s d}=\$ 2,260 \\
\mathbf{v}=5,108,987\end{array}$ & $\begin{array}{c}\mu=\$ 1,164 \\
s d=\$ 2,658 \\
v=7,063,389\end{array}$ \\
\hline $\begin{array}{l}\text { COSTl at C } \\
\text { Immediate Post-Intervention }\end{array}$ & $\begin{array}{c}\mu=\$ 716 \\
\mathbf{s d}=\$ 3,108 \\
\mathbf{v}=9,659,044\end{array}$ & $\begin{array}{c}\mu=\$ 340 \\
\text { sd }=\$ 661 \\
\mathbf{v}=436,583\end{array}$ & $\begin{array}{c}\mu=\$ 355 \\
s d=\$ 619 \\
v=382,714\end{array}$ \\
\hline $\begin{array}{l}\text { COST1 at D } \\
2 \text { Months Post-Intervention }\end{array}$ & $\begin{array}{c}\mu=\$ 1,245 \\
s d=\$ 5,952 \\
v=35,421,687\end{array}$ & $\begin{array}{c}\mu=\$ 455 \\
\mathbf{s d}=\$ 848 \\
\mathbf{v}=718,970\end{array}$ & $\begin{array}{c}\mu=\$ 489 \\
s d=\$ 749 \\
v=561,561\end{array}$ \\
\hline $\begin{array}{l}\text { COST1 at E } \\
4 \text { Months Post-Intervention }\end{array}$ & $\begin{array}{c}\mu=\$ 1,308 \\
s d=\$ 4,792 \\
v=22,967,641\end{array}$ & $\begin{array}{c}\mu=\$ 408 \\
s d=\$ 943 \\
v=888,414\end{array}$ & $\begin{array}{c}\mu=\$ 568 \\
s d=\$ 1,187 \\
v=1,408,381\end{array}$ \\
\hline
\end{tabular}


Another method of portraying utilization costs across time uses confidence intervals. By establishing a confidence interval, the degree of confidence that program outcomes will offset program implementation costs can be shown. The confidence interval variable (CONFINT) equals program outcomes as defined in the net benefit equation. Because this aggregate variable requires complete health care utilization data at points B (immediate pre-), C (immediate post-), D (2-months post-), and E (4-months post-), the majority of cases are excluded from this analysis. CONFINT does, however, provide another way of evaluating the cost-benefit of the Living Well workshop, and provides a useful analysis tool for future efforts. Table 20 shows the confidence intervals (at $95 \%, 80 \%$, and $75 \%$ ) associated with program outcomes for the cohort, trimmed, and high-use data.

Table 20: Program Outcome Confidence Intervals

\begin{tabular}{||c|c|c|c||}
\hline Confidence Intervals & $\begin{array}{c}\text { Cohort Data } \\
\mathbf{n}=93 \\
\text { Median }=-89\end{array}$ & $\begin{array}{c}\text { Trimmed Data } \\
\mathbf{n}=69 \\
\text { Median }=0\end{array}$ & $\begin{array}{c}\text { High Users (Trimmed) } \\
\mathbf{n}=48 \\
\text { Median }=184\end{array}$ \\
\hline $\begin{array}{c}\text { 95\% C.I. of CONFINT } \\
\text { Lower Bound } \\
\text { Upper Bound }\end{array}$ & $\begin{array}{c}\$-2,119 \\
\$ 2,148\end{array}$ & $\begin{array}{c}\$-198 \\
\$ 1,589\end{array}$ & $\begin{array}{c}\$ 73 \\
\$ 2,549\end{array}$ \\
\hline $\begin{array}{c}\text { 80\% C.I. of CONFINT } \\
\text { Lower Bound } \\
\text { Upper Bound }\end{array}$ & $\$-1,372$ & $\$ 116$ & $\$ 511$ \\
\hline $75 \%$ C.I. of CONINT \\
Lower Bound \\
Upper Bound & $\$ 1,404$ & $\$ 1,275$ & $\$ 2,110$ \\
\hline
\end{tabular}

Using cohort group data, the confidence intervals are extremely large and do not provide much security for positive intervention outcomes. This result, however, is less 
discouraging for the trimmed data set and for high medical care utilizers. Within the first six months of the Living Well intervention, medical cost decreases occur at the $80 \%$ and $75 \%$ confidence interval when outliers have been excluded, and this result is extended to the $95 \%$ confidence interval when examining high medical care users. If the Living Well intervention can target participants who have high medical care use, significant reductions in medical costs are probable. In fact, considering the $\$ 596$ per person cost of implementing the Living Well workshop, insurance companies can be confident at the $75 \%$ level that they will see a positive return on their investment within the first 6 months for high medical care users.

Analysis of confidence intervals would be more fruitful if the data were more complete across time. Construction of the CONFINT variable resulted in $50.5 \%$ of cohort cases, $63.3 \%$ of trimmed cases, and $60 \%$ of high cost user cases to be excluded from analysis. Obviously, these rates of exclusion provide a large degree of uncertainty. For the remainder of this analysis, individual means for COST1 at points $B, C, D$, and E are used to maintain a higher percentage of valid cases. It should be noted, however, that these means introduce measurement error since total health care changes are derived from different sub-populations.

Analysis of confidence intervals for each point of cost data provides an interesting graphical representation of how health care costs change. The following three charts show confidence intervals at B (immediate pre-intervention), C (immediate post-), D (2months post-), and $\mathrm{E}$ (4 months post-) for the cohort, trimmed, and high cost subsamples. 
The graphical representations show how the $95 \%$ confidence intervals for health care costs decrease from the baseline (B) to subsequent data collection points $(C, D$, and E). Additionally, the confidence intervals are smaller after the baseline, yielding more certain outcomes. The trimmed and high medical use data samples produced much smaller confidence intervals for all data points, which adds to the confidence of cost outcomes. 
Chart 1: 95\% Confidence Intervals for Cohort Data

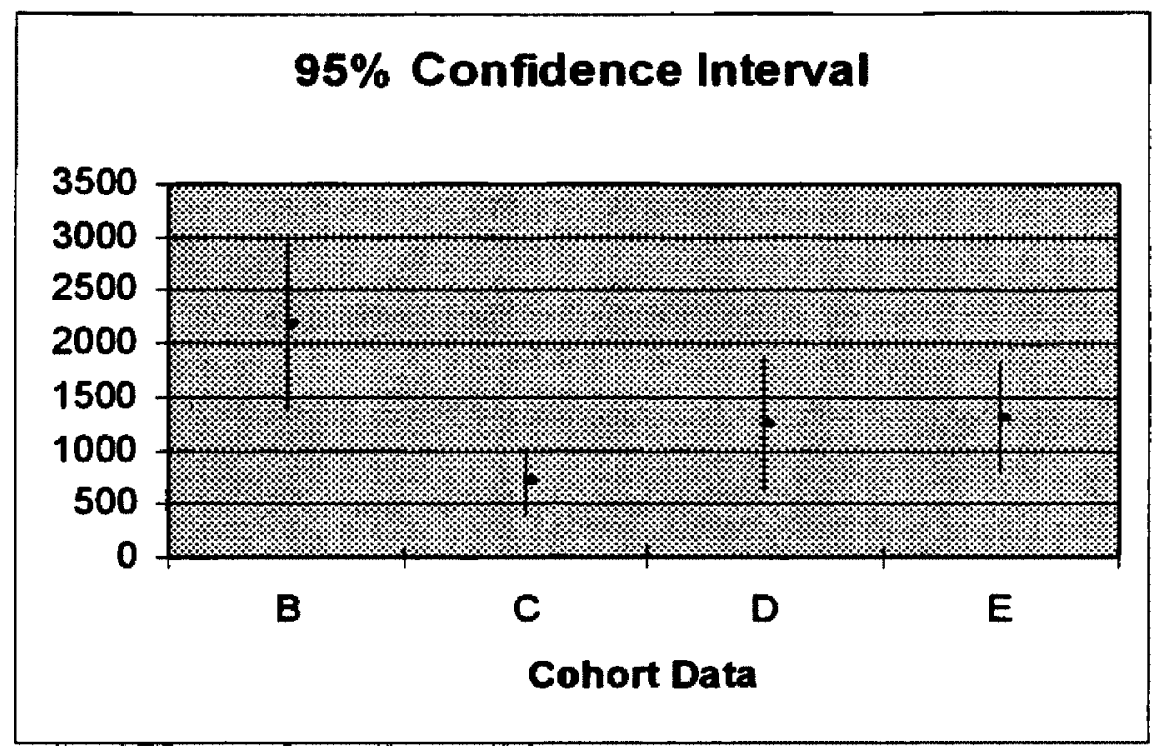

Chart 2: 95\% Confidence Intervals for Trimmed Data

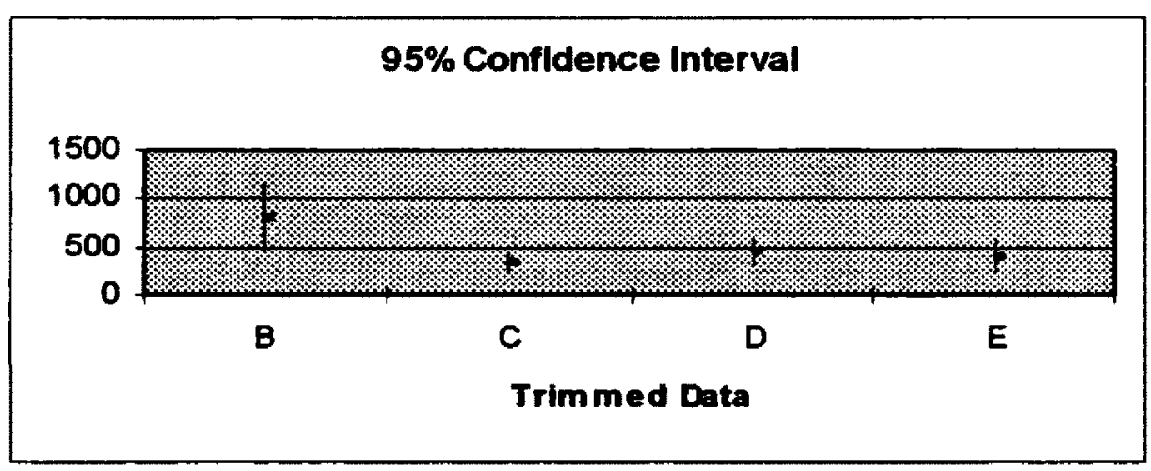

Chart 3: 95\% Confidence Intervals for High Medical Use Data

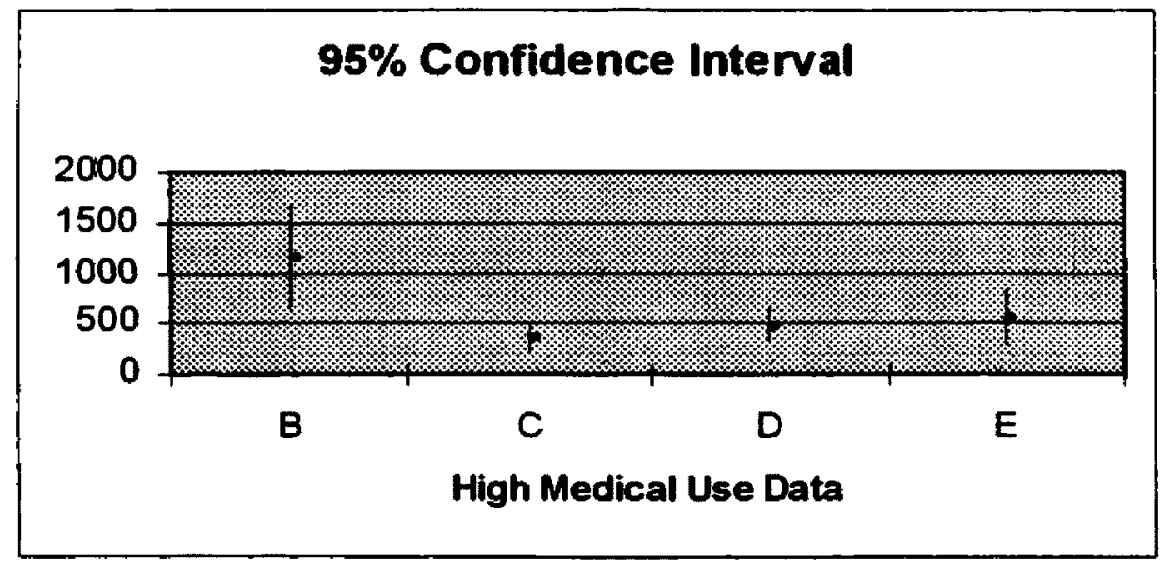

71 


\section{Section 3: Quality of Life Changes}

Although this study focuses on the cost benefits of the Living Well intervention, non-quantifiable quality of life indicators were impacted by the intervention as well. For this study, these indicators include the sum of secondary conditions, the total health promoting lifestyle score, a general life satisfaction variable, and overall quality of life. As described in previous sections, the sum of secondary conditions is the arithmetic sum of ratings across 43 secondary conditions. The total Health Promoting Lifestyle score measures attributes in six different areas of healthy living. The life satisfaction variable measures general life satisfaction on a scale of 1 (very satisfied) to 4 (very dissatisfied), and the quality of life variable measures overall quality of life on a scale of 0 (worst possible quality of life) to 10 (best quality of life). Table 21 shows the mean value changes for each of the quality of life indicators. For each measure, the mean values move in the direction of enhanced health outcomes - sum of secondary conditions decrease over time, health promoting lifestyle characteristics increase, general life satisfaction moves towards more satisfaction, and quality of life improves. 
Table 21: Quality of Life Indicators

\begin{tabular}{|c|c|c|c|c|c|}
\hline Sample & $\begin{array}{c}\text { B } \\
\text { immediate } \\
\text { pre - }\end{array}$ & $\begin{array}{c}\mathrm{C} \\
\text { immediate } \\
\text { post - }\end{array}$ & $\begin{array}{c}\text { D } \\
\text { two } \\
\text { months } \\
\text { post - }\end{array}$ & $\begin{array}{c}E \\
\text { four } \\
\text { months } \\
\text { post - }\end{array}$ & $\begin{array}{c}F \\
\text { one year } \\
\text { post - }\end{array}$ \\
\hline \multicolumn{6}{|l|}{ cohort } \\
\hline Sum of Secondary Conditions & $\begin{array}{c}29.84 \\
n=188\end{array}$ & $\begin{array}{c}27.05 \\
n=188\end{array}$ & $\begin{array}{c}26.18 \\
n=166\end{array}$ & $\begin{array}{c}24.12 \\
\mathrm{n}=157\end{array}$ & $\begin{array}{c}24.65 \\
\mathrm{n}=117\end{array}$ \\
\hline Total Health Promoting Lifestyle & $\begin{array}{c}2.49 \\
\mathrm{n}=175\end{array}$ & $\begin{array}{c}2.56 \\
\mathrm{n}=178\end{array}$ & $\begin{array}{c}2.59 \\
\mathrm{n}=158\end{array}$ & $\begin{array}{c}2.60 \\
\mathrm{n}=149\end{array}$ & $\begin{array}{c}2.58 \\
\mathrm{n}=110\end{array}$ \\
\hline General Life Satisfaction & $\begin{array}{c}2.20 \\
\mathrm{n}=182\end{array}$ & $\begin{array}{c}2.04 \\
n=186\end{array}$ & $\begin{array}{c}2.05 \\
\mathrm{n}=165\end{array}$ & $\begin{array}{c}2.01 \\
n=153\end{array}$ & $\begin{array}{c}1.96 \\
n=116\end{array}$ \\
\hline Overall, Quality of Life & $\begin{array}{c}6.58 \\
n=179\end{array}$ & $\begin{array}{c}6.76 \\
\mathbf{n}=186\end{array}$ & $\begin{array}{c}6.68 \\
n=164\end{array}$ & $\begin{array}{c}6.83 \\
n=155\end{array}$ & $\begin{array}{c}6.94 \\
n=155\end{array}$ \\
\hline \multicolumn{6}{|l|}{ High Medical Use } \\
\hline Sum of Secondary Conditions & $\begin{array}{c}31.83 \\
\mathrm{n}=123\end{array}$ & $\begin{array}{c}28.92 \\
\mathrm{n}=123\end{array}$ & $\begin{array}{c}28.39 \\
\mathrm{n}=105\end{array}$ & $\begin{array}{c}25.16 \\
\mathrm{n}=101\end{array}$ & $\begin{array}{l}25.64 \\
n=74\end{array}$ \\
\hline Total Health Promoting Lifestyle & $\begin{array}{c}2.54 \\
\mathrm{n}=116\end{array}$ & $\begin{array}{c}2.62 \\
\mathrm{n}=116\end{array}$ & $\begin{array}{c}2.65 \\
\mathrm{n}=98\end{array}$ & $\begin{array}{c}2.67 \\
\mathrm{n}=97\end{array}$ & $\begin{array}{c}2.67 \\
\mathrm{n}=71\end{array}$ \\
\hline General Life Satisfaction & $\begin{array}{c}2.19 \\
\mathrm{n}=121\end{array}$ & $\begin{array}{c}2.05 \\
\mathrm{n}=122\end{array}$ & $\begin{array}{c}2.07 \\
\mathrm{n}=104\end{array}$ & $\begin{array}{c}2.05 \\
\mathrm{n}=98\end{array}$ & $\begin{array}{c}1.99 \\
\mathrm{n}=74\end{array}$ \\
\hline Overall, Quality of Life & $\begin{array}{c}6.49 \\
\mathrm{n}=121 \\
\end{array}$ & $\begin{array}{c}6.75 \\
\mathrm{n}=122 \\
\end{array}$ & $\begin{array}{c}6.68 \\
\mathrm{n}=103 \\
\end{array}$ & $\begin{array}{c}6.85 \\
\mathrm{n}=101 \\
\end{array}$ & $\begin{array}{c}6.88 \\
\mathrm{n}=74 \\
\end{array}$ \\
\hline
\end{tabular}

Table 22 shows paired sample t-tests for the cohort and high-medical-use subsample. Paired samples are shown for immediate pre-intervention (B) to four post intervention points in time ( $\mathrm{C}$ - immediate post, $\mathrm{D}-2$ months post, $\mathrm{E}-4$ months post, and F- 1 year post-intervention) for the stated quality of life indicators. 
Table 22: Quality of Life Indicators - Equality of Means Tests

\begin{tabular}{|c|c|c|c|c|c|c|}
\hline Paired Sample & $\begin{array}{c}\text { Cohort } \\
\mathbf{t} \\
\end{array}$ & $\begin{array}{c}\text { Cohort } \\
\text { df }\end{array}$ & $\begin{array}{c}\text { Cohort } \\
\text { Sig } \\
\text { (2-tailed) } \\
\end{array}$ & $\begin{array}{c}\text { High-Use } \\
t\end{array}$ & $\begin{array}{l}\text { High-Use } \\
\text { df }\end{array}$ & $\begin{array}{l}\text { High-Use } \\
\text { Sig } \\
\text { (2 tailed) }\end{array}$ \\
\hline \multicolumn{7}{|l|}{$\begin{array}{l}\text { Sum of Secondary } \\
\text { Conditions }\end{array}$} \\
\hline $\mathrm{C}$ to $\mathrm{B}$ & 3.614 & 187 & $0.000 * *$ & 3.211 & 122 & $0.003 * *$ \\
\hline $\mathrm{D}$ to $\mathrm{B}$ & 4.033 & 165 & $0.000 * *$ & 3.194 & 104 & $0.002 * *$ \\
\hline $\mathrm{E}$ to $\mathrm{B}$ & 4.573 & 156 & $0.000 * *$ & 4.746 & 100 & $0.000 * *$ \\
\hline$F$ to $B$ & 2.867 & 116 & $0.005 * *$ & 2.501 & 73 & $0.015^{*}$ \\
\hline \multicolumn{7}{|c|}{ Health Promoting L ifestyle } \\
\hline $\mathrm{C}$ to $\mathrm{B}$ & -3.846 & 167 & $0.000 * *$ & -3.590 & 110 & $0.000 * *$ \\
\hline $\mathrm{D}$ to $\mathrm{B}$ & -3.662 & 146 & $0.000 * *$ & -2.941 & 92 & $0.004 * *$ \\
\hline$E$ to $B$ & -3.239 & 139 & $0.002 * *$ & -3.323 & 92 & $0.001 * *$ \\
\hline$F$ to $B$ & -1.800 & 100 & 0.075 & -1.619 & 66 & 0.110 \\
\hline \multicolumn{7}{|l|}{ General Life Satisfaction } \\
\hline $\mathrm{C}$ to $\mathrm{B}$ & 3.606 & 179 & $0.000 * *$ & 2.657 & 119 & $0.009 * *$ \\
\hline $\mathrm{D}$ to $\mathrm{B}$ & 3.405 & 160 & $0.001 * *$ & 2.606 & 102 & $0.011 *$ \\
\hline$E$ to $B$ & 2.843 & 148 & $0.005 * *$ & 1.554 & 96 & 0.123 \\
\hline$F$ to $B$ & 3.938 & 113 & $0.000 * *$ & 2.335 & 73 & $0.022^{*}$ \\
\hline \multicolumn{7}{|c|}{ Overall Quality of Life } \\
\hline $\mathrm{C}$ to $\mathrm{B}$ & -1.819 & 177 & 0.071 & -2.262 & 119 & $0.026^{*}$ \\
\hline $\mathrm{D}$ to $\mathrm{B}$ & -0.435 & 154 & 0.664 & -1.224 & 100 & 0.224 \\
\hline$E$ to $B$ & -0.767 & 147 & 0.444 & -1.188 & 99 & 0.238 \\
\hline $\mathrm{F}$ to $\mathrm{B}$ & -1.244 & 110 & 0.216 & -0.670 & 72 & 0.505 \\
\hline
\end{tabular}

* significant at the 05 level

** significant at the .01 level

Despite any questions about the cost outcomes of the Living Well intervention, quality of life changes are demonstrably strong. Significant differences between pre- and post-intervention data are evident in the sum of secondary conditions, total health 
promoting lifestyle, and general life satisfaction variables. Although pre- to postintervention changes are not significant for overall quality of life, mean values move in a direction of enhanced quality of life. It appears that participants are healthier, have improved lifestyle attributes, and are more satisfied with their lives after participating in Living Well.

\section{Recommendations}

The preceding analysis shows the net benefits associated with participation in the Living Well health promotion program for individuals with mobility impairments.

Despite huge variances in cost data, the Living Well workshop was shown to pay for itself within the first two months for the study cohort. More conservative analyses, which excluded outliers more than three standard deviations from the mean, resulted in positive net benefits within four months of program implementation for medium cost data. The sub-sample of high-medical care users had significant cost differences at both the immediate post and 2-months post data collection points, and showed positive net benefits during this fourth month span. Additionally, confidence intervals reveal smaller cost ranges, and subsequently more confidence about cost expenditures after the Living Well intervention. These results are further strengthened by quality of life changes for the study cohort.

Clearly, the Living Well intervention impacts a range of health outcomes that extend beyond health care utilization. At the very least, this analysis has provided concrete information to third party payers about the attractiveness of supporting health promotion activities for individuals with mobility impairments. The bottom line is a 
significant payback for insurance carriers including low cost (Medicaid), medium cost (Medicare) and high cost (private insurance) reimbursement schedules in addition to enhanced quality of life for program participants. 


\section{Chapter 5: Discussion}

This thesis presented a net benefit analysis of the Living Well with a Disability health promotion program from the perspective of a third party payer. Although medical expenditures were particularly volatile between survey collection points, the Living Well program resulted in positive net benefits for all cost reimbursement schedules including low (Medicaid), medium (Medicare), and high (private insurance) medical reimbursement rates after the first six months of program implementation. Efficacy data also demonstrated programmatic strengths as participants reported significantly fewer secondary conditions, more health promoting lifestyle attributes, and a higher rate of general life satisfaction.

While these results are encouraging for widespread adoption of health promotion programs, the study has limitations which should be addressed. Primarily, the survey instrument and intervention design raise particular concerns. The following discussion speaks to these weaknesses and offers suggestions for future studies in this field.

\section{The Survey Instrument}

The Living Well survey instrument collected data about a range of subjects including secondary conditions, health care utilization measures, health status, depression, lifestyle, quality of life, and barriers to participation. Collecting data about this range of information resulted in a survey close to thirty pages in length. At the time

the survey instrument was developed, the efficacy of Living Well was still in question and it was important to explore several facets of behavior change in addition to health care utilization. Unfortunately, the sheer length of the data collection instrument resulted in 
the exclusion of several questions which would have better addressed a cost-benefit analysis from the societal perspective.

These questions include information about a range of indirect costs incurred by participants, including foregone wages and out-of-pocket expenses to participate in the Living Well intervention; lost time, wages, transportation, and attendant expenses associated with medical care utilization; and other indirect health outcomes, such as improved employment opportunities or reduced family stress. Without including the costs borne by program participants, the full impacts of the Living Well intervention are lost.

Additionally, the survey questions provide very little insight about the type of medical attention received. Hospital visits can range from intensive acute medical care to standard overnight procedures. Outpatient surgeries can include local anesthesia versus a full general anaesthesia, and emergency room visits can be construed as immediate care facilities like extended hour clinics versus in-hospital emergency visits. Obviously, there is information about services and associated costs which is lost when asking broad health utilization questions.

Survey construction was also weak in the area of tests and services. The test and therapy questions were often left missing and, in cases when information was completed, there was no avenue to indicate if services were independent of, or in conjunction with, a doctor, emergency room, hospital or outpatient visit. A close look at the data showed significant overlap between long hospital stays and high rates of tests and therapies. In certain cases, like the average daily hospital rate, this overlap resulted in double counting 
of test and therapy costs. This problem was addressed in the current study by excluding tests and therapies from analysis. In the future, additional information could be gained if these questions were clarified.

Uncertainties regarding health utilization are compounded by Medicare cost estimates. Average unit costs may misrepresent the mix of services actually received by program participants. For instance, the unit cost for a doctor's visit collapses numerous medical designations (internist $v$. podiatrist $v$. general practitioner $v$. oncologist) that have large disparities in unit costs. A misunderstanding about the mix of services required by a study population with mobility impairments could significantly change unit cost approximations.

\section{Other Survey Problems}

In addition to weaknesses in the survey construction, the analysis was compromised because of missing data, missing surveys, and potential reporting biases. Missing data and significant attrition across data points resulted in paired comparisons which, in some instances, excluded more than half of the cohort. At the time of this study, of the 188 participants who completed both a B (immediate pre-) and C (immediate post-) measure, only 115 completed an F (1 year post-) measure. When survey instruments were returned, large segments of data were frequently missing. Obviously, the degree of missing information negatively impacts the confidence about study findings.

Self-reported medical care utilization data is also a concern. Lorig, et al. (1998) estimates that individuals underestimate service utilization by $17 \%$. If medical 
utilization was consistently underestimated in the Living Well sample, program outcomes may have been under-reported. Conversely, it is possible that the Hawthorne effect could have played a role in inflating program outcomes if participants overstated reductions in medical care utilization.

\section{Intervention Design}

The intervention design was compromised by the absence of a true control group which makes it difficult to differentiate between intervention impacts and outside factors such as health and environmental trends. This is particularly true for individuals who have degenerative conditions that require increased health utilization across time (Kinne, Patrick, \& Maher, 1999). No change in medical care utilization does not necessarily indicate no intervention outcome. In the absence of a control measure, however, this effect is difficult to measure. Although an extended baseline can address questions about the stability of health care utilization, the Living Well extended baseline covered only a two-month interval. Given a relatively small sample size coupled with huge cost variances, this two month span could not adequately answer questions about health care utilization changes across time.

Finally, recruitment levels were a problem. Although the Living Well intervention was designed to serve up to 12 participants per replication, workshops had an average of six to seven participants. This elevated per person costs to participate in the Living Well intervention and potentially reduced program net benefits.

\section{Recommendations for Future Studies}

Responding to limitations outlined in this discussion, future cost-benefit studies 
could be improved. First, a revised survey instrument which adequately addresses the societal impacts of the Living Well intervention would augment the third-party payer perspective. As outlined in the US Public Health Service recommendations (1996), a societal perspective is important for informing decisions when different perspectives are not comparable (Russell, Gold, Siegel, Daniels, \& Weinstein, 1996). Particularly at the policy level, a societal perspective is vital to informed decision making.

As part of this survey revision, clearer and more specific questions regarding medical care utilization could help inform how the Living Well intervention impacts health. Not only can specific medical questions aid in accurately estimating unit costs, they can also show how medical utilization trends change in response to an intervention. For instance, the distinction between increases in preventative doctor's visits versus acute care visits is an important consideration for health promotion.

Data collection efforts could be improved to reduce attrition across the study horizon. Follow-up phone interviews or more assertive follow-up could be used to enhance response rates across time. Additionally, access to medical claims data could be used to supplement missing data and compare how self-report health care utilization tracks actual paid claims. Finally, a longer time horizon in conjunction with a larger study sample and true control group is required for a more complete picture of intervention outcomes.

Refining the Living Well survey instrument and design provides a platform to more adequately address questions of longer term health outcomes and net benefits from a variety of perspectives. In the mean time, this study provides a clear message to third 
party payers about health promotion for individuals with mobility impairments - positive program net benefits within six months of Living Well implementation. 


\section{Reference List}

Aldana, S., Jacobson, B., Harris, C., Kelley, P., \& Stone, W. (1993). Influence of a mobile worksite health promotion program on health care costs. American Journal of Preventative Medicine, 9 378-383.

Andrich, R., Ferrario, M., \& Moi, M. (1998). A model of cost-income analysis for assistive technology. Disability and Rehabilitation, 20, 1-24.

Barry, P. \& DeFriese, G. (1990). Cost-benefit and cost-effectiveness analysis for health promotion programs. American Journal of Health Promotion, 4, 448-452.

Beaves, R., Joesph, H., Rohrer, J., \& Zeitler, R. (1988). Cost-effectiveness: How it should be determined? Evaluation and the Health Professions, 11, 213-230.

Becker, H., Stuifbergen, A., \& Sands, D. (1991). Development of a scale to measure barriers to health promotion activities among persons with disabilities. American Journal of Health Promotion, 5, 449-454.

Bertera, R. (1993). Behavioral risk factor and illness day changes with workplace health promotion: Two year results. American Journal of Health Promotion, 7, 365-373.

Birch, S. \& Donaldson, C. (1987). Applications of cost-benefit analysis to health care: departures from welfare economic theory. Journal of Health Economics, 6, 211-225.

Bowne, D., Russell, M., Morgan, J., Optenberg, S., \& Clarke, A. (1984). Reduced disability and health care costs in an industrial fitness program. Journal of Occupational 
Medicine, 26, 809-816.

Consumer Price Index (2000, October 18). Retrieved January 17, 2000, from the World Wide Web: http://stats.bls.gov/cpihome.htm.

Cronan, T., Hay, M., Groessl, E., Bigatti, S., Gallagher, R., \& Tomita, M. (1998). The effects of social support and education on health care costs after three years. Arthritis Care and Research, 11, 326-334.

Donaldson, C. (1990). The state of the art of costing health care for economic evaluation. Community Health Studies, 14, 341-356.

Dor, A. \& Farley, D. (1996). Payment source and the cost of hospital care: Evidence from a multiproduct cost function with multiple payers. Journal of Health Economics, $15,1-21$.

Drummond, M., Stoddart, G., \& Torrance, G. (1987). Methods for the economic evaluation of health care programmes. Oxford: Oxford University Press.

Edwards, P. (1996). Health promotion through fitness for adolescents and young adults following spinal cord injury. SCI Nursing, 13, 69-73.

Epstein, A., Hall, J., Tognetti, J., Son, J., \& Conant, L. (1989). Using proxies to evaluate quality of life. Medical Care, 27, S91-S98.

French, A. \& Poulsen, J. Multivariate analysis of variance. 12-17-1999. Ref Type: Unpublished Work 
Fries, J., Harrington, H., Edwards, R., Kent, L., \& Richardson, N. (1994).

Randomized controlled trial of cost reductions from a health education program: The California Public Employee's Retirement System (PERS) study. American Journal of Health Promotion, 8, 216-223.

Fryback, D. G., Dasbach, E., Kline, R., Klein, B.E., Dorn, N., \& Peterson, K. (1993). The beaver dam outcomes study: Initial catalog of health-state quality factors. Med.Decis.Making. 13, 89-102.

Garber, A. \& Phelps, C. (1997). Economic foundations of cost-effectiveness analysis. Journal of Health Economics, 16, 1-31.

Golaszewski, T., Lynch, W., Clearie, A., \& Vickery, D. (1989). The relationship between retrospective health claims and a health risk appraisal-generated measure of health status. Joumal of Occupational Medicine, 31, 262-264.

Gold, M., Siegel, J., Russell, L., \& Weinstein, M. (Eds.). (1996). Costeffectiveness in health and medicine. New York: Oxford University Press.

Guo, J., Gibson, T., Gropper, D., Oswald, S., \& Barker, K. (1998). Empiric investigation on direct costs-of-illness and healthcare utilization of Medicaid patients with Diabetes Mellitus. The American Journal of Managed Care, 4 1433-1446.

Haddix, A., Teutsch, S., Shaffer, P.A., \& Dunet, D. (Eds.). (1996). Prevention effectiveness: A guide to decision analysis and economic valuation. New York: Oxford 
University Press.

Hadorn, D. \& Uebersax, J. (1995). Large-scale health outcomes evaluation: How should quality of life be measured? Part 1 - calibration of a brief questionnaire and a search for preference subgroups. Journal of Clinical Epidemiology 48, 607-618.

Hinman, A. (1999). Economic aspects of vaccines and immunizations. Life Sciences, 322, 989-994.

Hoffman, C., Rice, D., \& Sung, H. (1996). Persons with chronic conditions: Their prevalence and costs. The Joumal of the American Medical Association, 276, 1473-1480.

Husted, C., Pham, L., Hekking, A., \& Niederman, R. (1999). Improving quality of life for people with chronic conditions: the example of $\mathrm{T}^{\prime}$ ai $\mathrm{Chi}$ and multiple sclerosis. Alternative Therapies, 5, 70-74.

Kaman, R. \& Patton, R. (1992). Costs and benefits of an active versus and inactive society. In (pp. 134-144).

Keeler, E., Manning, W., Newhouse, M., Sloss, E., \& Wasserman, J. (1989). The external costs of a sedentary life-style. American Journal of Public Health. 79, 975-981.

Kinne, S., Patrick, D., \& Maher, E. (1999). Correlates of exercise maintenance among people with mobility impairments. Disability and Rehabilitation, 21, 15-22.

Kirk, R. (1995). Experimental design: Procedures for the behavioral sciences. (3 ed.) Brooks/Cole Publishing Company. 
Kruger, J., Helmick, C., Callahan, L., \& Haddix, A. (1998). Cost-effectiveness of the arthritis self-help course. Archives of Internal Medicine, 158, 1245.

Leveille, S., Wagner, E., Davis, C., Grothaus, L., Wallace, J., LoGerfo, M., \& Kent, D. (1998). Preventing disability and managing chronic conditions in frail older adults: A randomized trial of a community-based partnership with primary care. Journal of American Geriatric Society, 46, 1198.

Lorig, K., Gonzalez, V., \& Ritter, P. (1999). Community-based Spanish language arthritis education program. Medical Care, 37, 957-963.

Lorig, K., Gonzales, D., Laurent, L., \& Laris, B. (1998). Arthritis selfmanagement program variations: Three studies. The American College of Rheumatology 11 448-454.

Lorig, K., Sobel, D., Stewart, A., Brown, B., Bandura, A., Ritter, P., Gonzalez, V., Laurent, D., \& Holman, H. (1999). Evidence suggesting that a chronic disease selfmanagement program can improve health status while reducing hospitalization. Medical Care, 37, 5-14.

Maher, E., Kinne, S., \& Patrick, D. (1999). Finding a good thing: The use of quantitative and qualitative methods to evaluate an exercise class and promote exercise for adults with mobility impairments. Disability and Rehabilitation, 21, 438-447.

Marge, M. (1988). Health promotion for personas with disabilities: Moving 
beyond rehabilitation. American Journal of Health Promotion, 2. 29-35.

Messonnier, M., Corso, P., Teutsch, S., Haddix, A., \& Harris, J. (1999). An Ounce of prevention....what are the returns? American Journal of Preventative Medicine 16 248-263.

Murray, C.J.L., Lopez, A.D. (Eds.). (1996). The global burden of disease: A comprehensive assessment of mortality and disability from diseases, injuries, and risk factors in 1990 and projected into 2020. Cambridge: World Health Organization.

Norton, S. (1995). Medicaid fees and the Medicare fee schedule: An update. Health Care Financing Review, 17, 167-181.

Norusis, M. (1999). SPSS 6.1: A guide to data analysis. (4 ed.) New Jersey: Prentice-Hall, Inc.

Pelletier, K. (1996). A review and analysis of the health and cost-effective outcome studies of comprehensive health promotion and disease prevention programs at the worksite: 1993-1995 update. American Journal of Health Promotion, 10, 380-388.

Phillips, K., Holtgrave, D. (1997). Using cost-effectiveness/cost-benefit analysis to allocate health resources: A level playing field for prevention. American Journal of Preventative Medicine, $13,18-25$

Radloff, L. (1977). The CES-D scale: A self-report depression scale in research in the general population. Applied Psychological Measurement, 1, 385-401. 
Ravesloot, C., Seekins, T., \& Quincy-Robyn Y. (1998). Health promotion for people with chronic illness and physical disabilities: The connection between health psychology and disability prevention. Clinical Psychology and Psychotherapy, 5, 75-85.

Ravesllot, C., Young, Q., Norris, K., Szalda-Petree, A., Seekins, T., Duffy, S., White, G., Lopez, C., Golden, K. (1998) Living well with a disability ( $2^{\text {nd }}$ ed.). Missoula: The University of Montana, Rural Institute on Disabilities.

Rimmer, J. (1999). Health promotion for people with disabilities: The emerging paradigm shift from disability prevention to prevention of secondary conditions. Physical Therapy, 79, 495-502.

Russell, L., Gold, M., Siegel, J., Daniels, N., \& Weinstein, M. (1996). The role of cost-effectiveness analysis in health and medicine. The Journal of American Medicine, 276. 1172 .

Sciacca, J., Seehafer, R., Reed, R., \& Mulvaney, D. (1993). The impact of participation in health promotion on medical costs: A reconsideration of the Blue Cross and Blue Sheild of Indiana study. American Journal of Health Promotion, 7, 374-383.

Seekins, T. \& Ravesloot, C. (2000). Secondary conditions experienced by adults with injury-related disabilities in Montana. Top Spinal Cord Injury Rehabilitation, 6 4353.

Shephard, R. (1989). Current perspectives on the economics of fitness and health 
with particular reference to worksite programs. Sports Medicine, 7, 286-309.

Shi, L. (1993). Health promotion, medical care use, and costs in a sample of worksite employees. Evaluation Review, 17, 475-487.

Stachtchenko, S. \& Jenicek, M. (1990). Conceptual differences between prevention and health promotion: research implications for community health programs. Canadian Journal of Public Health, 81, 53-59.

Stevens, W., Hillsdon, M., Thorogood, M., \& McArdle, D. (1998). Costeffectiveness of a primary care based physical activity intervention in 45-74 year old men and women: A randomized controlled trial. British Journal of Sports Medicine, 32, 236241.

Stoddard, S., Jans, L., Ripple, J. and Kraus, L. (1998). Chartbook on work and disability in the United States: 1998. An InfoUse Report. Washington, D.C.: U.S. National Institute on Disability and Rehabilitation.

Stoddart, G. (1982). Economic evaluation methods of health policy. Evaluation and the Health Professions, 5, 393-414.

Taylor, W., Baranowski, T., \& Young, D. (1998). Physical activity interventions in low-income, ethnic minority, and population with disability. American Journal of Preventative Medicine, 15, 334-343.

Thacker, S., Koplan, J., Taylor, W., Hinman, A., Katz, M., \& Roper, W. (1994). 
Assessing prevention effectiveness using data to drive program decisions. Public Health Reports, 109, 187-194.

U.S. Census Bureau. (1999, July 1). State and county quikfacts. Retrieved January 17,2000 , from the World Wide Web:

http://quickfacts.census.gov/qfd/index.html.

Walker, S., Sechrist, K., \& Pender, N. (1987). The health-promoting lifestyle profile: Development and psychometric characteristics. Nursing Research, 36, 78-81. 


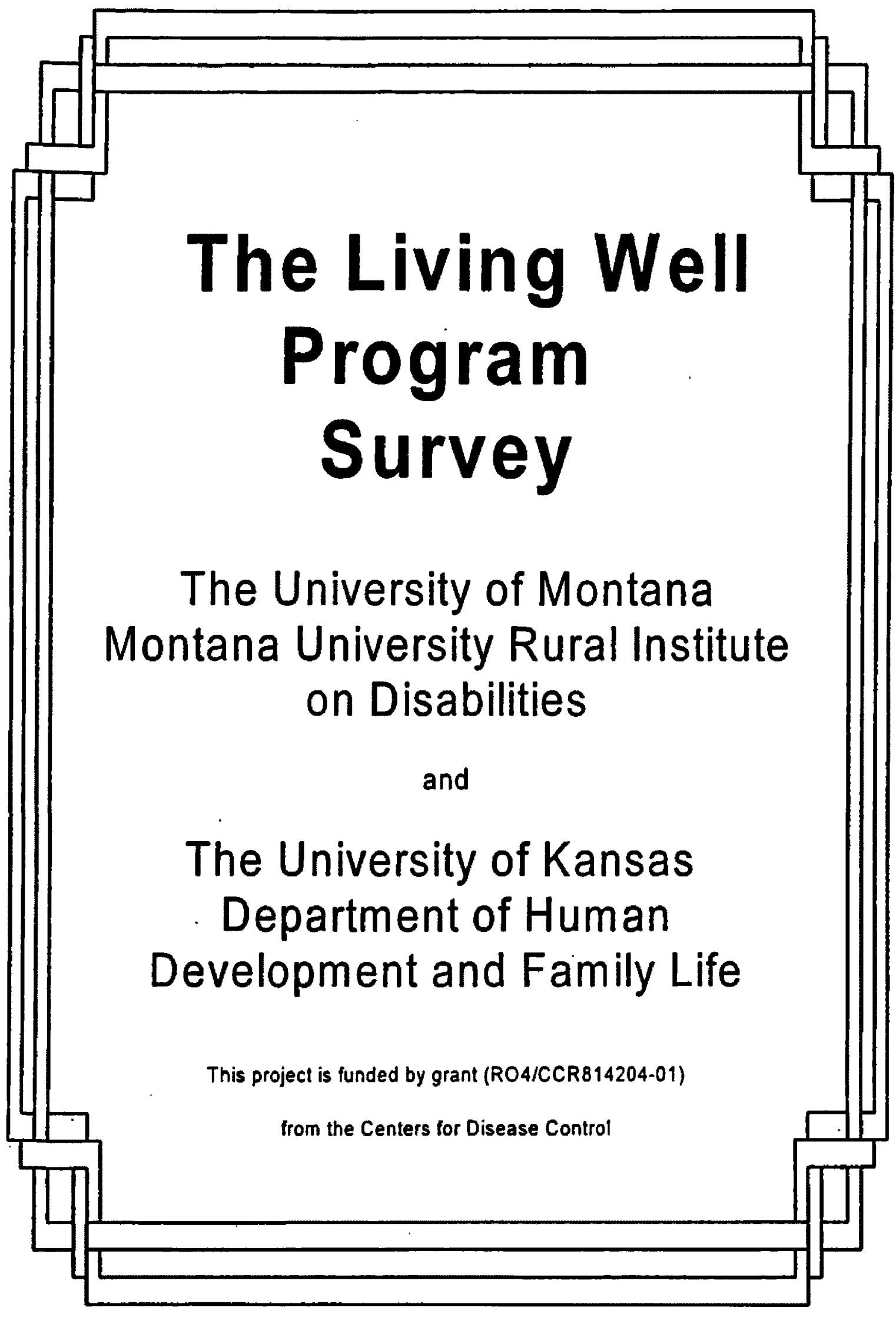

ID\#

92

Blue Survey - B 


\section{Dear Living Well Workshop Participant:}

We appreciate your involvement in the Living Well Program and will pay you $\$ 10$ each time you complete this survey. Over the next 18 months, we will ask you to complete this survey as many as six different times. We will pay you $\$ 10$ each time.

This research study is technically called a "cost-effectiveness study." To do the study, we need to know whether or not the Living Well Program was helpful to you and how the program may have affected your personal costs and your health care costs. To do this, the survey asks about your health, your income and your use of medical services in the last couple of months.

In order to use the information you give us in this survey, we need to have your permission to participate in this study. Please read and sign the Informed Consent and Permission to Contact form included in this survey. Everything you write in this survey will be kept strictly confidential. Your name should not appear anywhere in the survey. We will assign an ID number to this survey so that your name does not need to be on it. When we receive the survey, we will separate the Informed Consent/Permission Form from the rest of the survey to protect your confidentiality.

We anticipate that it may take you between 60 and 90 minutes to complete the survey and we very much appreciate your time and efforts. Please do your best to answer every question. If you are unsure of an answer, please try to find the correct information (e.g., how much your health insurance costs each month). If you cannot find the correct information, please answer with your best guess.

Our mission is to make this program and ones like it available to people with disabilities around the country. Your time and efforts filling out this survey (and the surveys to follow over the next 18 months) are vital to this mission. Again, thank you for your time.

Sincerely,

Living Well with a Disability Staff 


\section{DEMOGRAPHIC INFORMATION}

In order to better understand the health care needs of people with disabilities, we need to find out specific information about you.

\section{PERSONAL:}

Date of Birth:

Male

Female County of Residence:

Years of Education (Including 1st grade and beyond):

Number in household, including self:

Marital Status: Single Married Separated

RACE: White Black or African American Native Hawaiian or Other Pacific Islander Asian American Indian or Alaska Native

\section{ETHNICITY:}

Hispanic or Latino

Not Hispanic or Latino

EMPLOYMENT STATUS: (Check $\checkmark$ all that apply)

Not currently employed Retired
Employed part-time Homemaker Volunteer
Employed full-time Student

HEALTH CARE COVERAGE: (Check $\checkmark$ all that apply) Medicaid

- VA, CHAMPUS, CHAMP-VA Private health insurance Medicare Indian Health Service No health insurance 


\section{Primary Disability Information}

Please indicate the nature of your primary disability and approximate date of its diagnosis.

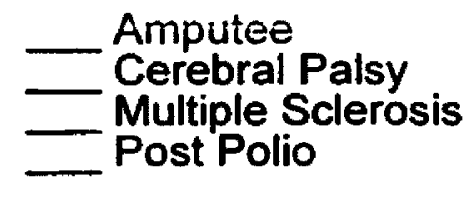
Arthritis
- Cardio-Pulmonary Disorders
- Muscular Dystrophy
- Spinal Cord Injury
Other (specify)

If you have a spinal cord injury, what is the level of your spinal cord injury (C1 - S5)?

Is your condition paraplegia or quadriplegia?

Is your injury complete or incomplete?

Approximate date of disability/ diagnosis:

\section{Overall Health and Independence}

Please rate your overall health and independence over the past two months.

Overall, would say your HEALTH over the past two months was:

Excellent Good Fair $\quad$ Poor

Overall, would you say your ability to INDEPENDENTLY engage in desired activities such as work, recreation or daily living over the past two months was:
Excellent
Good
Fair
Poor 
This survey gathers information about secondary conditions and other experiences related to health and disability, including emotional experiences, and general outlook. You may find it helpful to take at least one break between the sections. We understand that the survey is long, but we need to gather all of this information to learn what is most useful. Thank you in advance for your patience and effort.

\section{Secondary Conditions}

A secondary condition is a problem experienced after you have a primary disability. For example, a person with cerebral palsy may develop arthritis. Arthritis would then be a secondary condition for that person. Like a primary disability, a secondary condition may restrict your ability to do things independently.

Please rate how much each of the following conditions affected your activity and independence in the last two months. If you have not experienced a secondary condition in the last two months, or if it is an insignificant problem for you, please circle "0". Please refer to the rating scale, which is reproduced on each page, in making your ratings.
$0=$ Not experienced during past two months/insignificant problem (rarely or never limits activity or independence)
$1=$ Mild or infrequent problem (limits activity 1 to 5 hours per week)
2 = Moderate/occasional problem (limits activity 6 to 10 hours per week)
$3=$ Significant/chronic problem (limits activity 11 or more hours per week)

Injuries to the Skin/Body

0123 Pressure Sores

0123 Injuries Due to Loss of Sensation

0123 Care-related Injuries to yourself

0123 Amputation

\section{Description}

These develop as a skin rash or redness and may progress to an infected sore. Also called skin ulcers, bedsores, or decubitus ulcers. Persons who use wheelchairs are at risk for developing pressure sores.

Many people with disabilities involving loss of sensation (e.g., spinal cord injury, MS) report injuries because they can not feel pain in some areas (e.g., frostbite, burns from sitting too close to heater or fire).

When others provide personal care, some injuries can result, such as skin abrasions or a broken leg during a transfer.

Some individuals have had a limb or limbs removed for medical reasons. 
$0=$ Not experienced during past two months/insignificant problem (rarely or never limits activity or independence)

$1=$ Mild or infrequent problem (limits activity 1 to 5 hours per week)

$2=$ Moderate/occasional problem (limits activity 6 to 10 hours per week)

$3=$ Significant/chronic problem (limits activity 11 or more hours per week)

0123 Spasticity

(Muscle Spasms)

0123 Scoliosis (Kyphosis/Lordosis)

0123 Contractures

0123 Heterotopic Bone Ossification

0123 Osteoporosis

0123 Arthritis

0123 Fatigue
Spasticity refers to uncontrolled, jerky muscle movements, such as uncontrolled muscle twitch or spasm. Often spasticity increases with infection. Persons with multiple sclerosis, cerebral palsy, and spinal cord injury are among individuals at risk for developing spasticity.

These three terms refer to an abnormal curvature of the spine. Scoliosis is the curvature of the spine sideways. Lordosis is the forward curvature of the lower back. Kyphosis is the curvature of the upper back (hunchback). Persons with $\mathrm{SCl}$ are at risk of these because of not sitting right, muscle imbalance, or paralysis.

A contracture is a limitation in range of motion caused by shortening of the soft tissue around a joint (e.g., elbow, hips). This occurs when a joint can not move frequently enough through its range of motion. Pain commonly accompanies this condition.

This is an overgrowth of bone, often occurring after a fracture. Early signs include a loss in range of motion, local swelling, and warmth at the area to the touch. It must be diagnosed by a physician.

This is a wasting of bone. It may cause pain, can lead to fractures, and predisposes individuals to developing urinary tract stones. Any disabled individual who is not able to have adequate weight bearing exercise on their bones may develop osteoporosis, and women are at particular risk. It is diagnosed by a physician.

Arthritis results from inflammation of the joints, making movement both difficult and painful. Symptoms include pain and swelling around the joints. Cold weather and stress can make this condition worse.

Fatigue is a tired (though not necessarily sleepy) feeling after minimal exertion. 
$0=$ Not experienced during past two months/insignificant problem (rarely or never limits activity or independence)

$1=$ Mild or infrequent problem (limits activity 1 to 5 hours per week)

$2=$ Moderate/occasional problem (limits activity 6 to 10 hours per week)

$3=$ Significant/chronic problem (limits activity 11 or more hours per week)

\section{Weight/Physical Fitness Problems}

\section{Description}
0123 Physical Fitness or Conditioning Problems
0123 Eating or Weight Problems

\section{Bladder/Bowel Problems}

0123 Bladder Dysfunction

0123 Bowel Dysfunction

$\begin{array}{llllll}0 & 1 & 2 & 3 & \text { Urinary Tract }\end{array}$ Infections

0123 Sexual Dysfunction
Some disabled persons find they are not able to do as much as they would like because they are out of shape.

This includes difficulty in regulating weight, as well as problems with eating (e.g., overeating, under eating, vomiting food).

\section{Description}

Incontinence, bladder or kidney stones, kidney problems, leakage, urine backup, and associated problems are all symptoms of bladder dysfunction. Persons with impaired or absent muscle function in the area of the bladder are at risk for bladder dysfunction.

Diarrhea, constipation, "accidents," and associated problems are signs of bowel dysfunction. As with bladder dysfunction, persons with impaired muscle function or paralysis in the abdominal region are most likely to have bowel dysfunction.

This includes such infections as cystitis and pseudomonas. Symptoms include pain on urination, a burning sensation throughout the body, blood in the urine, and cloudy urine. Persons with multiple sclerosis and spinal cord injury are especially at risk for urinary tract infections.

This includes dissatisfaction with sexual functioning. Causes for dissatisfaction can be decreased sensation, changes in body image, difficulty in movement, and concern over bladder and bowel routines. 


$$
\begin{aligned}
0 & =\text { Not experienced during past two months/insignificant problem (rarely or never } \\
& \text { limits activity or independence) } \\
1 & =\text { Mild or infrequent problem (limits activity } 1 \text { to } 5 \text { hours per week) } \\
2 & =\text { Moderate/occasional problem (limits activity } 6 \text { to } 10 \text { hours per week) } \\
3 & =\text { Significant/chronic problem (limits activity } 11 \text { or more hours per week) }
\end{aligned}
$$

\section{Neurological Problems}

\section{Dysreflexia}
0123 Carpal Tunnel Syndrome

\section{Cardiovascular Problems}

0123 Postural Hypotension

\section{Cardiovascular (Heart) Problems}

0123 Circulatory Problems
This is a nerve disorder in the hand that causes pain and loss of feeling, especially in the thumb and first 3 fingers. Symptoms include numbness or tingling in part of the hand, shooting pains up the arm, thumb weakness, frequent dropping of objects, and shiny, dry skin on the hand.

Dysreflexia (sometimes called hyperreflexia) results from interference in the body's temperature and blood pressure regulating systems. Symptoms of dysreflexia include sudden rises in blood pressure and sweating, skin blotches, goose bumps, pupil dilation and headache. It is often related to overflowing leg bags. Dysreflexia can also occur as the body's response to pain where an individual doesn't experience sensation.

\section{Description}

This involves a strong sensation of lightheadedness following a change in position. It is caused by a sudden drop in blood pressure. Individuals with spinal cord injury or stroke may experience postural hypotension.

This commonly involves high or low blood pressure and must be diagnosed by a physician because there are often no symptoms. Other heart problems may be signaled by fluid retention - usually resulting in swelling around the ankles.

Swelling of veins, feet, or the occurrence of blood clots. Specify: 
$0=$ Not experienced during past two months/insignificant problem (rarely or never limits activity or independence)

$1=$ Mild or infrequent problem (limits activity 1 to 5 hours per week)

$2=$ Moderate/occasional problem (limits activity 6 to 10 hours per week)

$3=$ Significant/chronic problem (limits activity 11 or more hours per week)

\section{Respiratory}

0123 Respiratory Problems
Pneumonia and other respiratory tract infections can occur in disabled individuals. Symptoms of respiratory infections or problems include increased difficulty in breathing and increased secretions. Persons with quadriplegia, post polio, rheumatoid arthritis and multiple sclerosis are especially at risk for respiratory complications and infections.

\section{Description}

This is usually experienced as chronic tingling, burning or dull aches. It may occur in an area that normally has little or no feeling.

This includes pain in specific muscle groups or joints. Individuals who must overuse a particular muscle group (e.g., persons with paraplegia who may strain shoulder muscles) or those who must put too much strain on joints are at risk of developing joint and muscle pain.

\section{Description}

\section{Psychological}

0123 Depression

0123 Anger
More than feeling blue. Symptoms include: extreme, long-term sadness, loss of pleasure in favorite things and activities, difficulty sleeping, weight loss or gain, thoughts of suicide and frequent and/or unexplained crying.

Extreme displeasure with situations or persons that is difficult to forget. 
$0=$ Not experienced during past two months/insignificant problem (rarely or never limits activity or independence)

$1=$ Mild or infrequent problem (limits activity 1 to 5 hours per week)

$2=$ Moderate/occasional problem (limits activity 6 to 10 hours per week)

$3=$ Significant/chronic problem (limits activity 11 or more hours per week)

\section{Problems with Accessibility/Mobility Description}

0123 Isolation

$\begin{array}{llll}0 & 1 & 2 & \text { Problems with } \\ \text { Mobility }\end{array}$

0123 Access Problems

$\begin{array}{lllll}0 & 1 & 2 & 3 & \text { Equipment Failures }\end{array}$

$\begin{array}{llll}0 & 1 & 2 & 3 \\ \text { Equipment-related }\end{array}$ Injuries to Yourself

\section{Other Problems}

\section{Side Effects From Medications}

\section{Alcohol/Drug Abuse \\ 0123 Diabetes}

Isolation from social contact and support may be a problem for some individuals, and may be due to a loss of relationships or being house-bound.

Many physically disabled individuals are troubled by difficulty with getting around, due to a loss of strength or muscle control.

Access problems in the environment, such as lack of curb cuts or accessible buildings and restrooms, can pose an obstacle to functioning independently.

Equipment failures, such as a broken walker or brace, can limit independence by increasing the difficulty or prohibiting the completion of many desired activities.

The use of adaptive equipment can lead to injuries (e.g., injuries to one's underarms from poorly fitting crutches) that can limit an individual's completion of desired activities.

\section{Description}

Several medications prescribed for various problems may produce unwanted side effects. Please specify medication(s) or side effects :

This involves use of alcohol and/or drugs.

Diabetes is a problem resulting from irregularities in blood sugar levels. Symptoms include frequent urination and excessive thirst. This condition is diagnosed by a physician. Native American individuals and persons who are overweight are at higher risk for developing diabetes. 
$0=$ Not experienced during past two months/insignificant problem (rarely or never limits activity or independence)

$1=$ Mild or infrequent problem (limits activity 1 to 5 hours per week)

$2=$ Moderate/occasional problem (limits activity 6 to 10 hours per week)

$3=$ Significant/chronic problem (limits activity 11 or more hours per week)

0123 Communication Difficulties

0123 Written

Communication

Problems

0123 Anemia

0123 Visual Problems

0123 Hearing Impairment

$\begin{array}{llll}0 & 2 & 3 \text { Sleep Problems/ } \\ \text { Disturbances }\end{array}$

0123 Care-related Injuries to Others

0123 Equipment-related Injuries to Others
This includes difficulty talking due to a ventilator, speech problems and disorders, impaired muscle control around the mouth and other problems communicating with others.

Visually impaired persons and persons with reading disorders may be print handicapped, while others can't turn pages or hold books and magazines. Still others find it difficult to write or type because of their disability.

Anemia is a low level of iron in the blood and often occurs in conjunction with pressure sores. Symptoms include fatigue and low energy. This condition is diagnosed by a physician.

Significant loss of ability to see (e.g., loss of acuity or field of vision) including blindness. Please specify the nature of your visual problems:

Difficulties with hearing in general, or of particular kinds of sounds, is the criteria for hearing impairment. Usually this condition is diagnosed by a specialist.

Difficulty falling asleep or staying asleep, difficulty staying awake during the day, or waking up early are all sleep disturbances.

Injuries to others can occur in the process of providing care, such as a sprained back that occurs while transferring someone.

The use of adaptive equipment can lead to injuries to others, such as injuries received moving heavy adaptive equipment. 


\section{THIS SECTION IS ABOUT YOUR USE OF HEALTH CARE SERVICES}

IN THE LAST TWO MONTHS

(the past 8 weeks from today)

\section{HOSPITAL STAYS}

In the last two months, have you been admitted as an inpatient to a hospital (meaning you stayed overnight)? Yes No

If yes, how many days, in total, were you in the hospital?

\section{EMERGENCY ROOM VISITS}

In the last two months, have you been treated in a hospital emergency room? Yes No

If yes, how many times?

\section{HOSPITAL OUTPATIENT SERVICES}

In the last two months, have you received treatment in a hospital for outpatient surgeries or other procedures without staying overnight? Yes No

If yes, how many times? 


\section{DOCTOR'S OFFICE SERVICES}

In the last two months, have you visited a physician in his/her office (include all types of medical doctors, such as General Practitioners, Family Practice Physicians, Internists, Physiatrists, Ophthalmologists, Neurologists, Psychiatrists, Gynecologists, Cardiologists, Osteopaths, Rheumatologists)? Yes No

If yes, how many total visits did you have in the past 2 months?

For the first 3 of these visits, please fill in the information for each visit in the table below.

\begin{tabular}{|l|l|l|l|}
\hline & Visit 1 & Visit 2 & Visit 3 \\
\hline $\begin{array}{l}\text { What was the reason for the } \\
\text { appointment? }\end{array}$ & & & \\
\hline $\begin{array}{l}\text { What was your travel time to get } \\
\text { there? }\end{array}$ & & & \\
\hline $\begin{array}{l}\text { How many minutes did you wait for } \\
\text { the Doctor? }\end{array}$ & & & \\
\hline $\begin{array}{l}\text { How many minutes were you with } \\
\text { the Doctor? }\end{array}$ & & & \\
\hline $\begin{array}{l}\text { Were you satisfied with the visit? } \\
\text { (circle one) }\end{array}$ & Yes Mostly Some No & Yes Mostly Some No & Yes Mostly Some No \\
\hline
\end{tabular}




\section{TESTS, SERVICES AND TREATMENTS}

Please complete this table for the following health care services, if you have received them in the past two months.

\begin{tabular}{|l|l|l|l|}
\hline TESTS: & $\begin{array}{l}\text { Number of times in } \\
\text { the past 2 months } \\
\text { you have had: }\end{array}$ & $\begin{array}{l}\text { What was your } \\
\text { travel time to } \\
\text { get there? }\end{array}$ & $\begin{array}{l}\text { How many } \\
\text { minutes did the } \\
\text { service take? }\end{array}$ \\
\hline Blood Test & & & \\
\hline Urine Test & & & \\
\hline EKG (Electrocardiogram) & & & \\
\hline Blood Pressure & & & \\
\hline X-ray & & & \\
\hline Prostate Exam & & & \\
\hline Pap Smear & & & \\
\hline Mammogram & & & \\
\hline THERAPY \& \\
SERVICES:
\end{tabular}




\section{MEDICATIONS}

How many prescription drugs do you take now? (please circle one)
12
34
5
$6 \quad 7$
89
10
More than 10

Please check $\checkmark$ any of the following type of drugs that you are taking now. The first column lists prescription drugs and the second column lists "over-the-counter" drugs that you can buy without a prescription.

PRESCRIBED BY A DOCTOR Analgesic (like Darvon, Demerol)

Antacid

Antibiotic

Anticoagulant (like Heparin)

Antidepressant (like Efexor)

Anti-inflammatory Agent (like Cortizone)

Antispasmodic

Arthritis Medication

Dermatologicals or Skin Wound Preparation

Diarrhea Medication (like Imodium)

Fiber Supplement

Hypolipidemic (like Mevacor, anticholesterol drugs)

Laxative

Muscle Relaxant

Pressure Sore Treatment

Sedative or Tranquilizer

Urinary Tract Agent

Vitamins
OVER-THE-COUNTER Analgesic (like Aspirin) Antacid Antibiotic Ointment

- Anti-inflammatory Agent Arthritis Medication Diarrhea Medication Fiber Supplement Laxative Pressure Sore Treatment Sedatives or Tranquilizer Vitamins

\section{PERSONAL ASSISTANCE}

Do you use paid Personal Care Assistance (PCA)? yes no

If yes, how many hours of paid Personal Care Assistance (PCA) did you use in the last month? hours

What is the method of payment for your PCA? (Please $\checkmark$ check all that apply)

$$
\begin{aligned}
& \text { Medicare/Medicaid } \\
& \text { Employer }
\end{aligned}
$$

Private Insurance Self Pay

Have you had any major changes in living arrangements (moved, a new room mate, bought a home) in the last 2 months? Yes No

If yes, what were they 


\section{COST OF MEDICAL CARE}

During the past month, about how much did you spend for medical care? Do not include the cost of over-the counter remedies, the cost of health insurance premiums or any costs for which you expect to be reimbursed.

Less than $\$ 500$

$\$ 500-\$ 1,999$

$\$ 2,000-\$ 2,999$

$\$ 3,000-\$ 4,999$

$\$ 5,000$ or more

During the past month, how much did you spend for health insurance premiums? Please include payroll deductions for premiums.

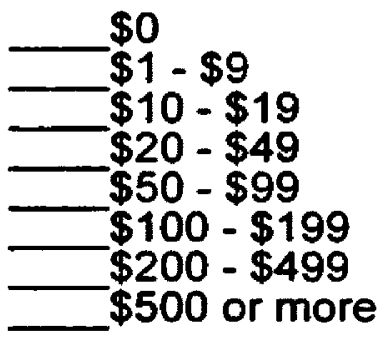

During the past month, how much did you spend for over-the-counter drugs that you can buy without a prescription.

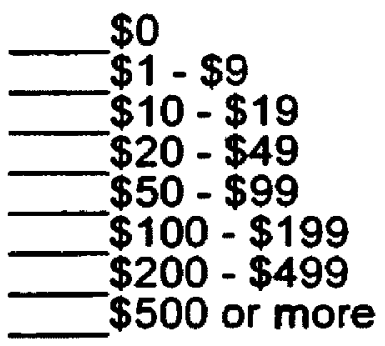




\section{INCOME}

1. What is the approximate combined monthly income of all family members in your household? (Consider all sources including wages and earnings, disability benefits, pensions and retirement income, social security, investments and trust funds, contributions from relatives and any other sources.)
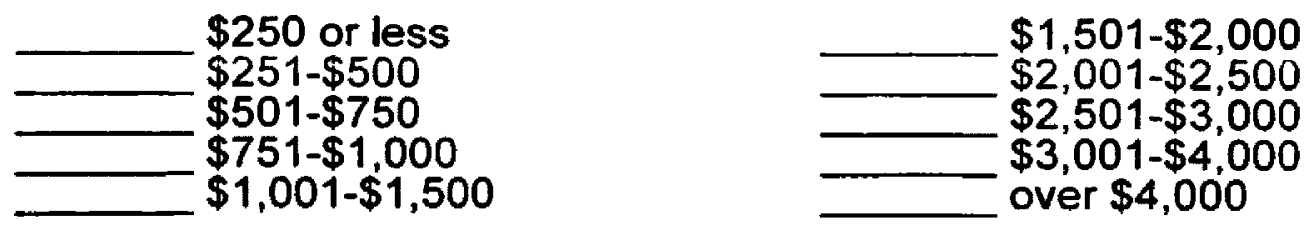

2. How much of your total family monthly income recorded in question 1 above is derived from your employment?
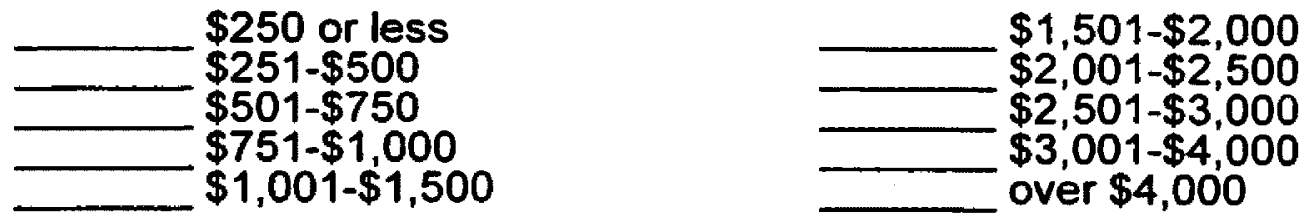

3. How much of your total family monthly income recorded in question 1 above is derived from Unemployment Insurance?
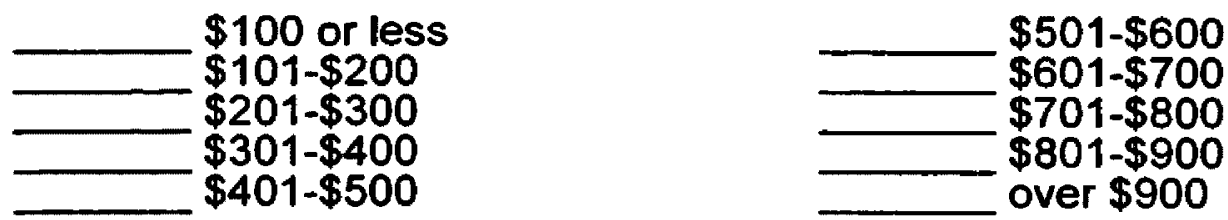

4. How much did you receive in SSDI or SSI payments in the past month?
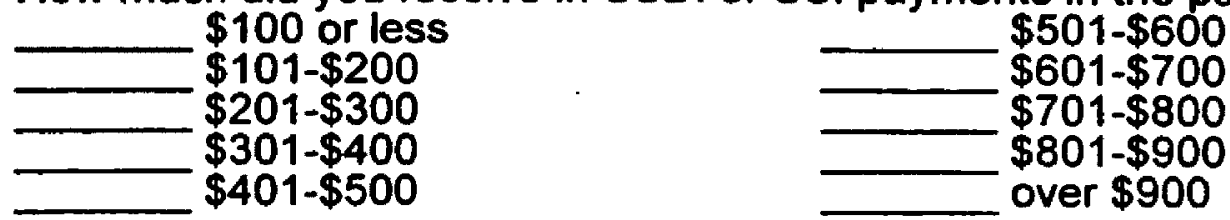

5. How much did you receive in General Assistance (i.e., food stamps, AFDC) payments in the past month?
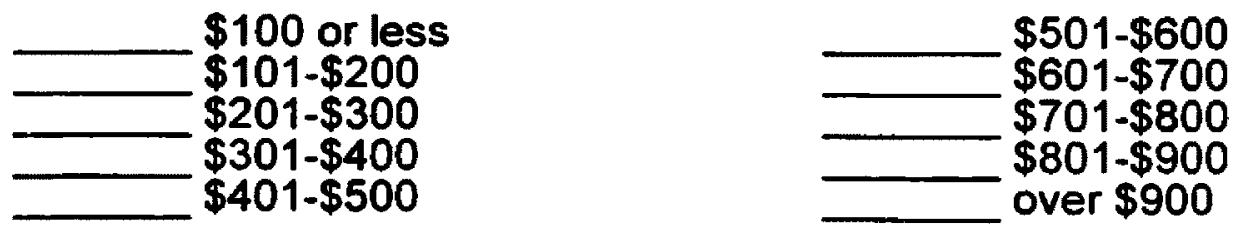


\section{HEALTH STATUS}

1. In general, how would you rate your health?

Excellent

Very good

Good

Fair

Poor

2. Thinking about your physical health, which includes physical illness and injury, for how many days during the past 30 days was your physical health not good?

(Please circle one number)

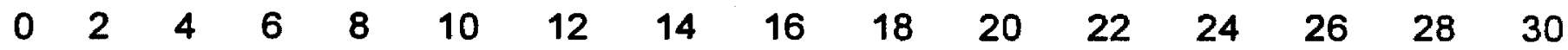

3. Thinking about your mental health, which includes stress, depression, and problems with emotions, for how many days during the past 30 days was your mental health not good? (Please circle one number)

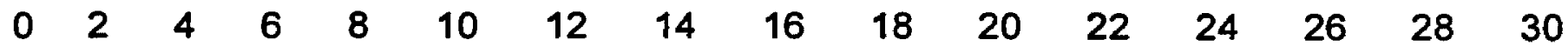

4. During the past 30 days, for about how many days did poor physical or mental health keep you from doing your usual activities, such as self-care, work, or recreation? (Please circle one number)

$\begin{array}{llllllllllllllll}0 & 2 & 4 & 6 & 8 & 10 & 12 & 14 & 16 & 18 & 20 & 22 & 24 & 26 & 28 & 30\end{array}$

THE NEXT TWO QUESTIONS ARE ABOUT YOUR SUPPORT NEEDS AND LIFE SATISFACTION.

5. How often do you get the social and emotional support you need?

Always Usually $\quad$ Sometimes $\quad \overline{\text { Rarely }}$ Never

6. In general, how satisfied are you with your life?

Very satisfied Satisfied Dissatisfied Very dissatisfied 


\section{THESE NEXT QUESTIONS ARE ABOUT LIMITATIONS YOU MAY HAVE IN YOUR DAILY LIFE}

7. Are you limited in the kind or amount of work you can do because of any impairment or health problem? Yes No

8. Because of any impairment or health problem, do you have any trouble learning, remembering, or concentrating? Yes No

9. If you use special equipment or help from others to get around, what type do you use? $(\checkmark$ all that apply)

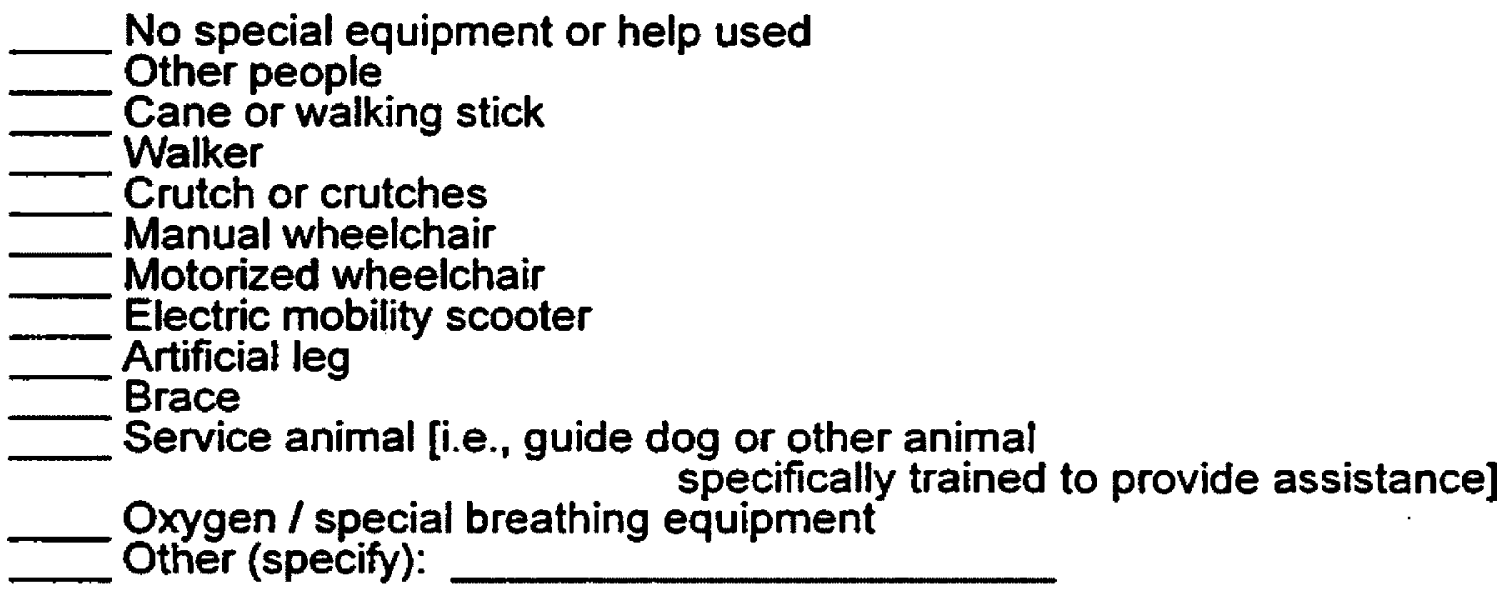

10. Using special equipment or help, what is the farthest distance that you can go? (Check $\checkmark$ one)

Across a small room About the length of a typical house About one or two city blocks About one mile More than one mile

11. What is the farthest distance you can walk by yourself, without any special equipment or help from others? (Check $\checkmark$ one)

Unable to walk

Across a small room

About the length of a typical house

About one or two city blocks

About one mile

More than one mile 
12. Are you limited in any way in any activities because of any impairment or health problem? Yes No

13. What is the MAJOR impairment or health problem that limits your activities?

(Check $\checkmark$ one)

Arthritis / rheumatism
Back or neck problem
Fractures, bone / joint injury
Walking problem
Lung / breathing problem
Learing problem
Hem $/$ vision problem
Eyem
Heart problem
Stroke problem
Hypertension / high blood pressure
Diabetes
Ciancer
Depression / anxiety / emotional problem
Oether impairment/problem

14. Since approximately what date have your activities been limited because of your major impairment or health problem?

month $\frac{1}{\text { year }}$

15. Because of any impairment or health problem, do you need the help of other persons with your PERSONAL CARE needs, such as eating, bathing, dressing, or getting around the house? Yes No

16. Because of any impairment or health problem, do you need the help of other persons in handling your ROUTINE NEEDS, such as everyday household chores, doing necessary business, shopping, or getting around for other purposes? Yes No

17. During the past 30 days, for about how many days did PAIN make it hard for you to do your usual activities, such as self-care, work, or recreation? (Please circle one number)
0
24
68
10
1214
$16 \quad 18$
20
$\begin{array}{llll}22 & 24 & 26 & 28\end{array}$

18. During the past 30 days, for about how many days have you felt SAD, BLUE, or DEPRESSED? (Please circle one number)

$\begin{array}{llllllllllllllll}0 & 2 & 4 & 6 & 8 & 10 & 12 & 14 & 16 & 18 & 20 & 22 & 24 & 26 & 28 & 30\end{array}$


19. During the past 30 days, for about how many days have you felt WORRIED. TENSE, or ANXIOUS? (Please circle one number)

$\begin{array}{llllllllllllllll}0 & 2 & 4 & 6 & 8 & 10 & 12 & 14 & 16 & 18 & 20 & 22 & 24 & 26 & 28 & 30\end{array}$

20. During the past 30 days, for about how many days have you felt that you did not get ENOUGH REST or SLEEP? (Please circle one number)

$\begin{array}{llllllllllllllll}0 & 2 & 4 & 6 & 8 & 10 & 12 & 14 & 16 & 18 & 20 & 22 & 24 & -26 & 28 & 30\end{array}$

21. During the past 30 days, for about how many days have you felt VERY HEALTHY and FULL OF ENERGY? (Please circle one number)

$\begin{array}{llllllllllllllll}0 & 2 & 4 & 6 & 8 & 10 & 12 & 14 & 16 & 18 & 20 & 22 & 24 & 26 & 28 & 30\end{array}$ 


\section{THIS SECTION IS ABOUT FEELINGS}

Circle the number for each statement which best describes how often you felt or behaved this way, during the past week. Use the following scale in your response:

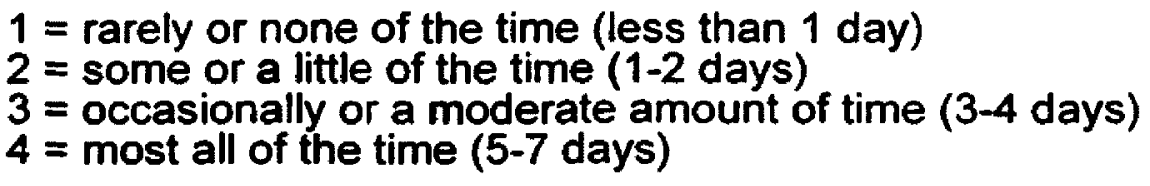

\section{During the Past Week:}

1. I was bothered by things that usually don't bother me.

2. I did not feel like eating: my appetite

$\begin{array}{llll}1 & 2 & 3 & 4\end{array}$
was poor.

3. I felt that I could not shake off the blues even with help from my family or friends.

4. I felt that I was just as good as other people.

$\begin{array}{llll}1 & 2 & 3 & 4\end{array}$

5. I had trouble keeping my mind on what I was doing.

$\begin{array}{llll}1 & 2 & 3 & 4\end{array}$

6. I felt depressed.

$\begin{array}{llll}1 & 2 & 3 & 4\end{array}$

7. I felt that everything I did was an effort.

$\begin{array}{llll}1 & 2 & 3 & 4\end{array}$

8. I felt hopeful about the future.

$\begin{array}{llll}1 & 2 & 3 & 4\end{array}$

9. I thought my life had been a failure.

$\begin{array}{llll}1 & 2 & 3 & 4\end{array}$


1 = rarely or none of the time (less than 1 day)

2 = some or a little of the time (1-2 days)

$3=$ occasionally or a moderate amount of time (3-4 days)

$4=$ most all of the time (5-7 days)

10. I felt fearful.

11. My sleep was restless.

12. I was happy.

13. I talked less than usual.

14. I felt lonely.

15. People were unfriendly.

16. I enjoyed life.

17. I had crying spells.

18. I felt sad.

19. I felt that people disliked me.

20. I could not get "going." $\begin{array}{llll}1 & 2 & 3 & 4\end{array}$

$\begin{array}{llll}1 & 2 & 3 & 4\end{array}$

$\begin{array}{llll}1 & 2 & 3 & 4\end{array}$

$\begin{array}{llll}1 & 2 & 3 & 4\end{array}$

$\begin{array}{llll}1 & 2 & 3 & 4\end{array}$

$\begin{array}{llll}1 & 2 & 3 & 4\end{array}$

$\begin{array}{llll}1 & 2 & 3 & 4\end{array}$

$\begin{array}{llll}1 & 2 & 3 & 4\end{array}$

$\begin{array}{llll}1 & 2 & 3 & 4\end{array}$

$\begin{array}{llll}1 & 2 & 3 & 4\end{array}$

$\begin{array}{llll}1 & 2 & 3 & 4\end{array}$ 


\section{THIS SECTION CONTAINS STATEMENTS ABOUT YOUR CURRENT WAY OF LIFE OR PERSONAL HABITS}

DIRECTIONS: Please respond to each item as accurately as possible, and try not to skip any item. How often do you do the following?

$\mathbf{N}$ for never, $\mathbf{S}$ for sometimes, $\mathbf{O}$ for often, or $\mathbf{R}$ for routinely

1. Discuss my problems and concerns with people close to me.

2. Choose a diet low in fat, saturated fat, and cholesterol.

3. Report any unusual signs or symptoms to a physician or other health professional.

4. Follow a planned exercise program.

5. Get enough sleep.

6. Feel I am growing and changing in positive ways.

7. Praise other people easily for their achievements.

8. Limit use of sugars and food containing sugar (sweets).

9. Read or watch TV programs about improving health.

10. Exercise vigorously for $\mathbf{2 0}$ or more minutes at least three times a week (such as brisk walking, bicycling, aerobic dancing, using a stair climber).

11. Take some time for relaxation each day.

12. Believe that my life has purpose.

13. Maintain meaningful and fulfilling relationships with others.

14. Eat 6-11 servings of bread, cereal, rice and pasta each day.

15. Question health professionals in order to understand their instructions.
$N \quad S \quad O \quad R$

$N$ S O R

$N$ S O R

N S O R

$N$ S O R

N S O R

$N$ S O R

N S O R

N S O R

N S O R

N $\quad S \quad O \quad R$

N $S$ O

N S O R

N S O R

$N \cdot S \quad O \quad R$ 
$\mathbf{N}$ for never, $\mathbf{S}$ for sometimes, $\mathbf{O}$ for often, Or $\mathbf{R}$ for routinely

16. Take part in light to moderate physical activity (such as sustained walking $30-40$ minutes

5 or more times a week).

17. Accept those things in my life which I cannot change.

$N$ S O R

18. Look forward to the future.

$N$ S O R

19. Spend time with close friends.

$N$ S O R

20. Eat 2-4 servings of fruit each day.

$N$ S O R

21. Get a second opinion when I question my health care provider's advice.

$N$ S O

22. Take part in leisure-time (recreational) physical activities (such as swimming, dancing, bicycling).

23. Concentrate on pleasant thoughts at bedtime.

24. Feel content and at peace with myself.

25. Find it easy to show concern, love and warmth to others.

$N$ S O $R$

26. Eat 3-5 servings of vegetables each day.

$N$ S O R

27. Discuss my health concerns with health professionals.

$N$ S O

28. Do stretching exercises at least 3 times per week.

$N$ S $\quad$ O $\quad R$

29. Use specific methods to control my stress.

$N$ S O R

30. Work toward long-term goals in my life.

$N$ S O $R$

31. Touch and am touched by people I care about.

$N$ S O $R$

32. Eat 2-3 servings of milk, yogurt or cheese each day.

$N$ S O R

33. Inspect my body at least monthly for physical changes/danger signs.

$N$ S $\quad$ O $\quad R$

$N$ S O R

$N$ S O R

N.S O R 
$\mathbf{N}$ for never, $\mathbf{S}$ for sometimes, O for often, Or $\mathbf{R}$ for routinely

34. Get exercise during usual daily activities (such as walking during lunch, using stairs instead of elevators, parking car away from destination and walking).

35. Balance time between work and play.

36. Find each day interesting and challenging.

$N$ S O R

37. Find ways to meet my needs for intimacy.

38. Eat only $2-3$ servings from the meat, poultry, fish, dried beans, eggs, and nuts group each day.

$N \quad S \quad O \quad R$

39. Ask for information from health professionals about how to take good care of myself.

40. Check my pulse rate when exercising.

41. Practice relaxation or meditation for 15-20 minutes daily.

$N$ S O $R$

42. Am aware of what is important to me in life.

$N$ S O $R$

$N$ S O R

$N \quad S \quad O \quad R$

43. Get support from a network of caring people.

$N$ S O R

44. Read labels to identify nutrients, fats, and sodium content in packaged food.

45. Attend educational programs on personal health care.

$N$ S O

46. Reach my target heart rate when exercising.

$N$ S O R

47. Pace myself to prevent tiredness.

$N$ S O

48. Feel connected with some force greater than myself.

$N$ S O

49. Settle conflicts with others through discussion and compromise.

$N$ S O

50. Eat breakfast.

$N$ S O

51. Seek guidance or counseling when necessary.

$N$ is O R

52. Expose myself to new experiences and challenges.

$N$ S O R 
INSTRUCTIONS: Please check the box $\nabla$ next to the word that best describes how you have been feeling over the past 2 months.

PHYSICAL SUFFERING: Headaches, chest or back pain, arthritis, nausea or vomiting, shortness of breath, dizziness, itching, etc.

NONE $\quad \square$ Physical suffering is rarely or never a problem.

MILD $\square$ Somewhat bothersome problem but generally goes away by itself.

MODERATE $\square$ More troubling problem with suffering.

SEVERE $\square$ Extremely disturbing problem with suffering.

EMOTIONSIOUTLOOK ON LIFE: Feeling happy or sad, peaceful or nervous, and how much you look forward to getting up in the morning. How much of a problem:

NONE $\square$ Emotions and outlook on life are rarely or never a problem.

MILD $\square$ Somewhat bothersome problem with feeling downhearted and blue.

MODERATE $\square$ More troubling problem with feeling depressed or nervous.

SEVERE $\square$ Extremely disturbing problem with feeling depressed or nervous.

DAILY ACTIVITIES: Working or favorite pastimes, doing things with friends and family, and basic self-care activities - such as: bathing, getting dressed, eating, and going to the bathroom. How much of a problem:

NONE $\square$ Daily activities are rarely or never a problem.

MILD $\square$ Somewhat bothersome problem with being limited in activities.

MODERATE $\square$ More troubling problem with having to reduce activities.

SEVERE $\square$ Extremely disturbing problem with having to reduce activities.

OVERALL, HOW WOULD YOU RATE YOUR QUALITY OF LIFE? (Circle one number)

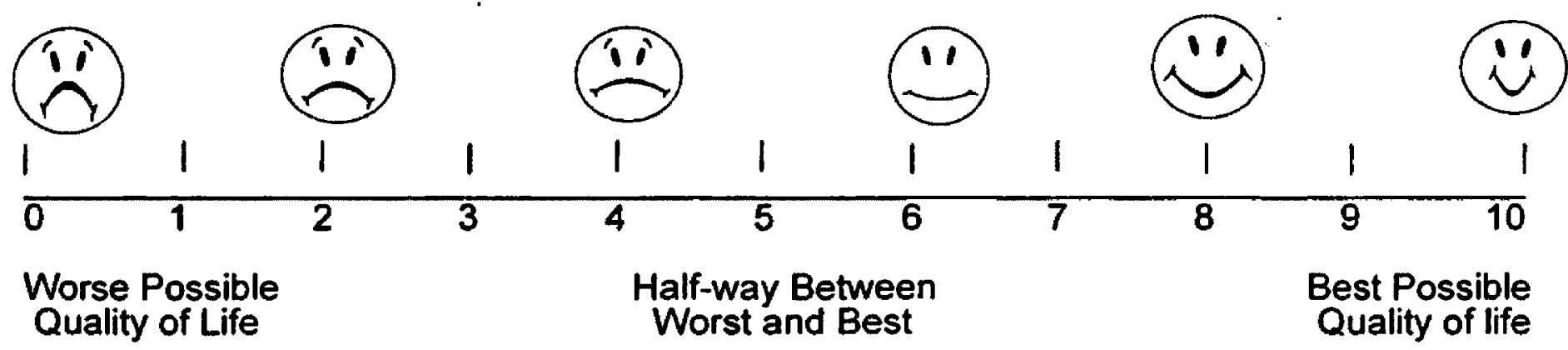




\section{POTENTIAL PROBLEMS WITH GOING TO EVENTS}

We are interested in how easy or difficult it will be for you to attend our health promotion program. The following is a list of things that could get in the way of coming to the Living Well workshop or similar events. For each statement, circle the number that represents how difficult each thing will make attending the program for you. If a statement does not apply to you or if it would not be a problem for you attending this program, please rate it a zero.

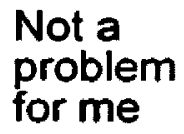

1. It's difficult to get in and out of my house.

2. My neighborhood has too few curb cuts.

3. It is dangerous for me to leave my house.

4. It would take too long to get to the program.

5. Chemicals in the environment bother me.

6. The weather is often too bad to get out.

7. I have trouble reading printed materials.

8. Buildings are not accessible to me.

9. I don't have accessible transportation.

10. I don't have the assistive equipment that I need.

11. My disability is limiting me too much these days.

12. I have a hard time thinking and concentrating.

13. I lose control over my bowel and bladder functions.

14. My weight makes it hard to get around.

15. I get tired easily.

16. I have pain when I do too much.

17. I can't see well enough to get around.

18. I have trouble hearing what people say.

20. I will have to take time off from my job.

$\begin{array}{llll}0 & 1 & 2 & 3\end{array}$

$\begin{array}{llll}0 & 1 & 2 & 3\end{array}$

$\begin{array}{llll}0 & 1 & 2 & 3\end{array}$

$\begin{array}{llll}0 & 1 & 2 & 3\end{array}$

$\begin{array}{llll}0 & 1 & 2\end{array}$

$\begin{array}{llll}0 & 1 & 2 & 3\end{array}$

$\begin{array}{llll}0 & 1 & 2 & 3\end{array}$

$\begin{array}{llll}0 & 1 & 2 & 3\end{array}$

$\begin{array}{llll}0 & 1 & 2 & 3\end{array}$

$\begin{array}{llll}0 & 1 & 2 & 3\end{array}$

$\begin{array}{llll}0 & 1 & 2 & 3\end{array}$

$\begin{array}{llll}0 & 1 & 2 & 3\end{array}$

$\begin{array}{llll}0 & 1 & 2 & 3\end{array}$

$\begin{array}{llll}0 & 1 & 2 & 3\end{array}$

$\begin{array}{llll}0 & 1 & 2 & 3\end{array}$

$\begin{array}{llll}0 & 1 & 2\end{array}$

$\begin{array}{llll}0 & 1 & 2 & 3\end{array}$

$\begin{array}{llll}0 & 1 & 2 & 3\end{array}$

$\begin{array}{llll}0 & 1 & 2 & 3\end{array}$


Not a
problem
for me

21. I'm too busy to take time away from other important activities.

22. I will have to arrange day care for my children.

23. I take care of another family member.

24. My family will not support my coming.

25. My daily self-care needs take too much energy.

26. I will need someone to help me.

27. My doctor will not approve of my coming.

28. Other important people will tell me not to come.
A very big problem for me

$\begin{array}{llll}0 & 1 & 2 & 3\end{array}$

$\begin{array}{llll}0 & 1 & 2 & 3 \\ 0 & 1 & 2 & 3 \\ 0 & 1 & 2 & 3 \\ 0 & 1 & 2 & 3 \\ 0 & 1 & 2 & 3 \\ 0 & 1 & 2 & 3 \\ 0 & 1 & 2 & 3\end{array}$


1998 Training Costs

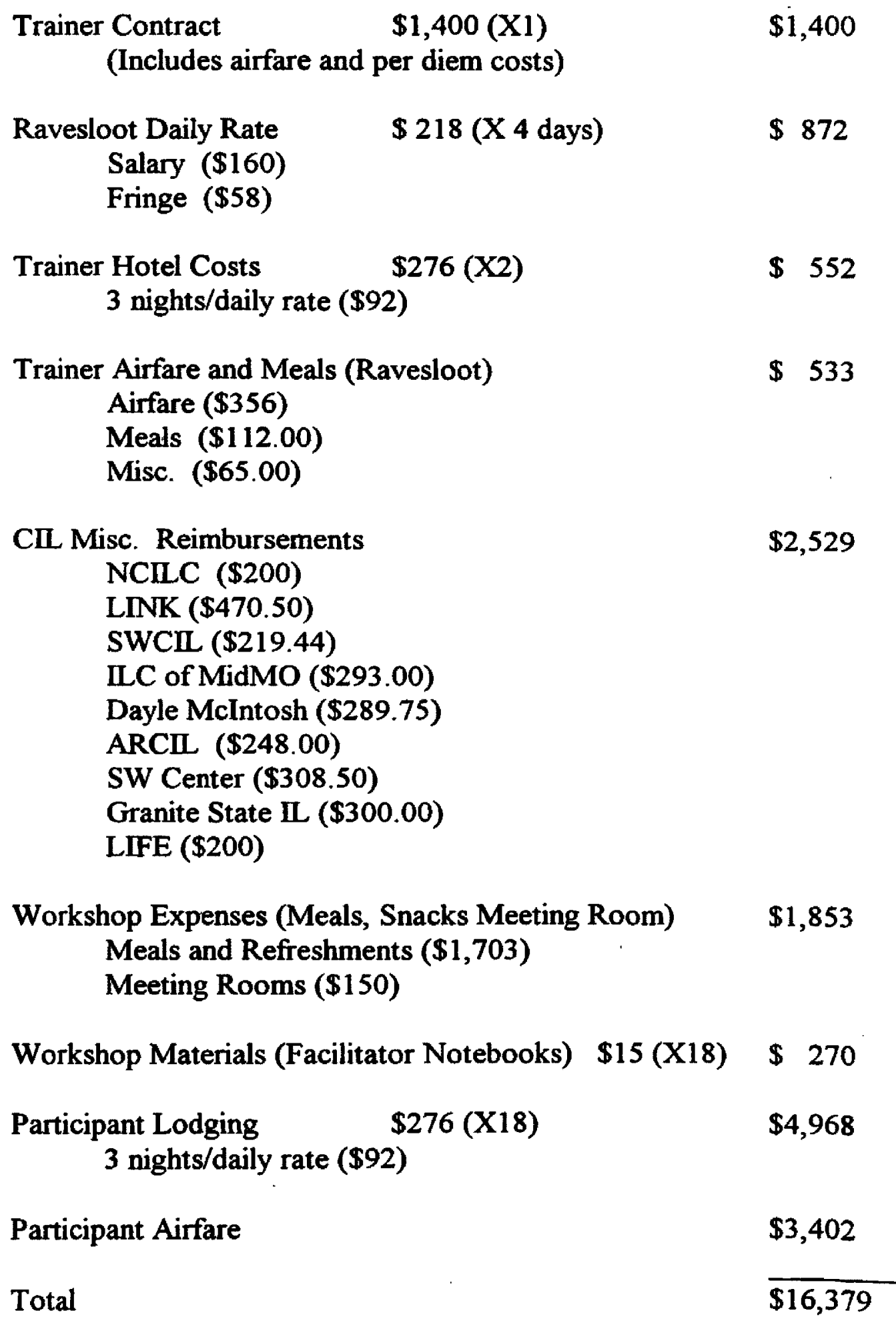


1999 Training Costs

Trainer Contract

$\$ 1,040(\mathrm{X} 1)$

$\$ 1,040$

(Includes airfare and per diem costs)

Ravesloot Daily Rate

\$ 276 (X 4 days)

$\$ 1,104$

Salary (\$204.17)

Fringe (\$71.86)

Trainer Hotel Costs

$\$ 360(X 2)$

$\$ 720$

3 nights/daily rate (\$120)

Trainer Airfare and Meals (Ravesloot)

$\$ 530$

Airfare (\$381)

Meals $(\$ 112.00)$

Misc. (\$37.00)

CIL Misc. Reimbursements

$\$ 1,294$

NCILC (\$97.99)

LINK (\$273.40)

SWCIL (\$289.75)

ILC of MidMO (\$273.50)

Dayle McIntosh (\$88.00)

ARCIL (\$271.00)

Workshop Expenses (Meals, Snacks Meeting Room) \$1,679

Equipment Rental (\$49)

Luncheons (\$898)

Break Refreshments (\$572.58)

Meeting Rooms (\$159.90)

Workshop Materials (Facilitator Notebooks) $\$ 15(\mathrm{X8)} \quad \$ 120$

Participant Lodging $\$ 276(X 8) \quad \$ 2,208$

3 nights/daily rate (\$92)

Participant Airfare

$\$ 1,454$

Total

$\$ 10,149$ 